\title{
Highly Efficient and Stable Vanadia-Titania-Sulfate Catalysts for Methanol Oxidation to Methyl Formate: Synthesis and Mechanistic Study
}

\author{
Ziyu Liu, ${ }^{a}$ Ruifang Zhang, ${ }^{a}$ Shibin Wang, ${ }^{\text {a,b }}$ Na Li, ${ }^{\text {acc }}$ Rui Sima, ${ }^{a}$ Guojuan Liu, ${ }^{a}$ Ping Wu, ${ }^{a}$ \\ Gaofeng Zeng, ${ }^{a}$ Shenggang Li, ${ }^{\mathrm{a}, \mathrm{b}, *}$ Yuhan $\mathrm{Sun}^{\mathrm{a}, \mathrm{b}, *}$
}

${ }^{a}$ CAS Key Laboratory of Low-Carbon Conversion Science and Engineering, Shanghai Advanced Research Institute, Chinese Academy of Sciences, 100 Haike Road, Shanghai 201210, China

${ }^{\mathrm{b}}$ School of Physical Science and Technology, ShanghaiTech University, 319 Yueyang Road, Shanghai 200031, China

${ }^{c}$ Department of Chemistry, Shanghai University, 99 Shangda Road, Shanghai 200444, China

Supporting Information Available: Additional Experimental Details. Complete Lists for References 10, 14, and 58. Figures: Additional TEM Images; $\mathrm{N}_{2}$ Adsorption-Desorption Isotherms; Alternative Pathways of the Hemiacetal Mechanism; Structures of the Stationary States on the PES. Tables: Surface Areas, Pore Sizes, Pore Volumes; V/Ti Atomic Ratios and Sulfur Contents Determined from Experiments; Data from Reaction Kinetic Studies; Fractional or Cartesian Coordinates of the Catalyst Models and the Stationary States on the PES. This material is available free of charge via the Internet at http://pubs.acs.org. 


\section{Additional Experimental Details}

The VTS catalysts were prepared by titrating the mixed solution of $4.0 \mathrm{~g}$ of $\mathrm{VOSO}_{4}, 17.4$ $\mathrm{g}$ of $\mathrm{Ti}\left(\mathrm{SO}_{4}\right)_{2}$ and $8.6 \mathrm{~g}$ of $\mathrm{H}_{2} \mathrm{SO}_{4}$ with the $\mathrm{NH}_{3} \cdot \mathrm{H}_{2} \mathrm{O}$ solution in an ice-water bath until the $\mathrm{pH}$ of the solution reached 9-10. The solution with the suspension was further stirred for 2 hours before being filtrated, washed and dried overnight at $120^{\circ} \mathrm{C}$. The catalysts were then calcinated in air for 5 hours at $350,400,450$, and $500^{\circ} \mathrm{C}$.

Powder X-ray diffraction (XRD) measurements were carried out with a Rigaku Ultima IV spectrometer using the $\mathrm{Cu} \mathrm{K \alpha}$ radiation $(\lambda=0.15406 \mathrm{~nm})$ operated at $40 \mathrm{kV}$ and $40 \mathrm{~mA}$ by scanning from 5 to $90^{\circ}(2 \theta)$ at a rate of $4^{\circ} / \mathrm{min}$.

Surface areas and pore sizes of the catalysts were measured by $\mathrm{N}_{2}$ adsorption at $77 \mathrm{~K}$ with Micromeritics TriStar II 3020, where the samples were degassed at $200^{\circ} \mathrm{C}$ for at least 2 hours under vacuum before the experiment. The specific surface area was calculated with the Brunauer-Emmett-Teller (BET) method, whereas the pore volume and pore size distribution were determined by the Barrett-Joyner-Halenda (BJH) method.

Transmission electron microscopy (TEM) measurements was carried out using a JEOL JEM-2100 microscope operated at an accelerating voltage of $200 \mathrm{kV}$. The samples were first dispersed in ethanol under ultrasonic conditions and a drop of the suspension was applied on the copper grid coated with the carbon film.

$\mathrm{NH}_{3}$ temperature programmed desorption (TPD) was recorded in a fix-bed reactor system equipped with a chromatograph. About $200 \mathrm{mg}$ of each catalyst was pretreated at $400^{\circ} \mathrm{C}$ under $\mathrm{Ar}$ flow $(28.5 \mathrm{~mL} / \mathrm{min})$ for 1 hour to remove water and other impurities. When the sample was cooled down to $100^{\circ} \mathrm{C}$ under Ar flow, $\mathrm{NH}_{3}(10 \mathrm{~mL} / \mathrm{min})$ was introduced for about $30 \mathrm{~min}$. Then the sample was swept using $\mathrm{Ar}$ at $100^{\circ} \mathrm{C}$ for about 40 min to remove the physisorbed $\mathrm{NH}_{3}$, the 
TPD spectra were recorded by increasing the temperature at a rate of $10^{\circ} \mathrm{C} / \mathrm{min}$ from $100^{\circ} \mathrm{C}$ to $600^{\circ} \mathrm{C}$.

The methanol oxidation reaction was carried out at atmospheric pressure in a fixed-bed microreactor made of stainless steel with an inner diameter of $8 \mathrm{~mm}$. All gas lines were kept at $100^{\circ} \mathrm{C}$ to prevent condensation of the reactants and products. Methanol was introduced into the vaporizer by a pump before mixing with the $\mathrm{O}_{2} / \mathrm{Ar}$ (1:9) mixture, and then the mixture passed through the reactor for the reaction. In each experimetn, $1.0 \mathrm{~g}$ of catalyst diluted with $1.0 \mathrm{~g}$ of quartz was loaded in the reactor, and the gas hourly space velocity (GHSV) was kept at 1160 $\mathrm{ml} \cdot \mathrm{g}_{\mathrm{cat}}{ }^{-1} \cdot \mathrm{h}^{-1}$. Reaction products were analyzed by on-line gas chromatography equipped with a Porapake-T column and a TCD detector. 


\section{Complete Lists for References 10, 14, and 58}

Ref. 10: Wang, R.; Wu, Z.; Chen, C.; Qin, Z.; Zhu, H.; Wang, G.; Wang, H.; Wu, C.; Dong, W.;

Fan, W.; Wang, J. Graphene-Supported Au-Pd Bimetallic Nanoparticles with Excellent Catalytic Performance in Selective Oxidation of Methanol to Methyl Formate. Chem. Commun. 2013, 49, 8250-8252.

Ref. 14: Whiting, G. T.; Kondrat, S. A.; Hammond, C.; Dimitratos, N.; He, Q.; Morgan, D. J.; Dummer, N. F.; Bartley, J. K.; Kiely, C. J.; Taylor, S. H.; Hutchings, G. J. Methyl Formate Formation from Methanol Oxidation Using Supported Gold-Palladium Nanoparticles. ACS Catal. 2015, 5, 637-644.

Ref. 58: Frisch, M. J.; Trucks, G. W.; Schlegel, H. B.; Scuseria, G. E.; Robb, M. A.; Cheeseman, J. R.; Scalmani, G.; Barone, V.; Mennucci, B.; Petersson, G. A.; Nakatsuji, H.; Caricato, M.; Li, X.; Hratchian, H. P.; Izmaylov, A. F.; Bloino, J.; Zheng, G.; Sonnenberg, J. L.; Hada, M.; Ehara, M.; Toyota, K.; Fukuda, R.; Hasegawa, J.; Ishida, M.; Nakajima, T.; Honda, Y.; Kitao, O.; Nakai, H.; Vreven, T.; Montgomery, J. A., Jr.; Peralta, J. E.; Ogliaro, F.; Bearpark, M.; Heyd, J. J.; Brothers, E.; Kudin, K. N.; Staroverov, V. N.; Kobayashi, R.; Normand, J.; Raghavachari, K.; Rendell, A.; Burant, J. C.; Iyengar, S. S.; Tomasi, J.; Cossi, M.; Rega, N.; Millam, N. J.; Klene, M.; Knox, J. E.; Cross, J. B.; Bakken, V.; Adamo, C.; Jaramillo, J.; Gomperts, R.; Stratmann, R. E.; Yazyev, O.; Austin, A. J.; Cammi, R.; Pomelli, C.; Ochterski, J. W.; Martin, R. L.; Morokuma, K.; Zakrzewski, V. G.; Voth, G. A.; Salvador, P.; Dannenberg, J. J.; Dapprich, S.; Daniels, A. D.; Farkas, Ö.; Foresman, J. B.; Ortiz, J. V.; Cioslowski, J.; Fox, D. J. Gaussian 09, Revision C.01, Gaussian, Inc., Wallingford CT, 2009. 
Figure S1. TEM Images of the (a) VTS-350, (b) VTS-400, (c) VTS-450, and (d) VTS-500 Catalysts.
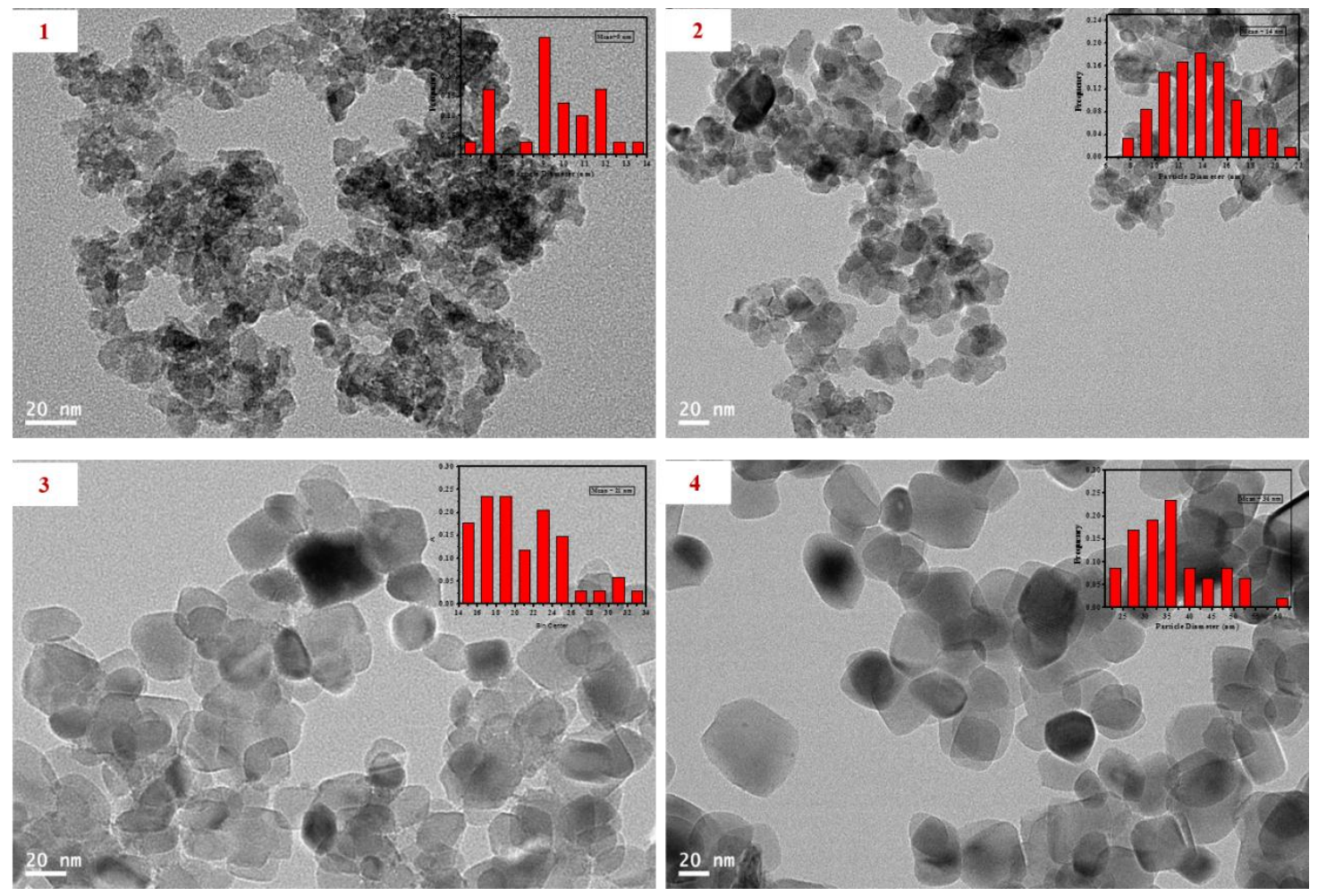

Figure S2. $\mathrm{N}_{2}$ Adsorption-Desorption Isotherms of the VTS Catalysts.

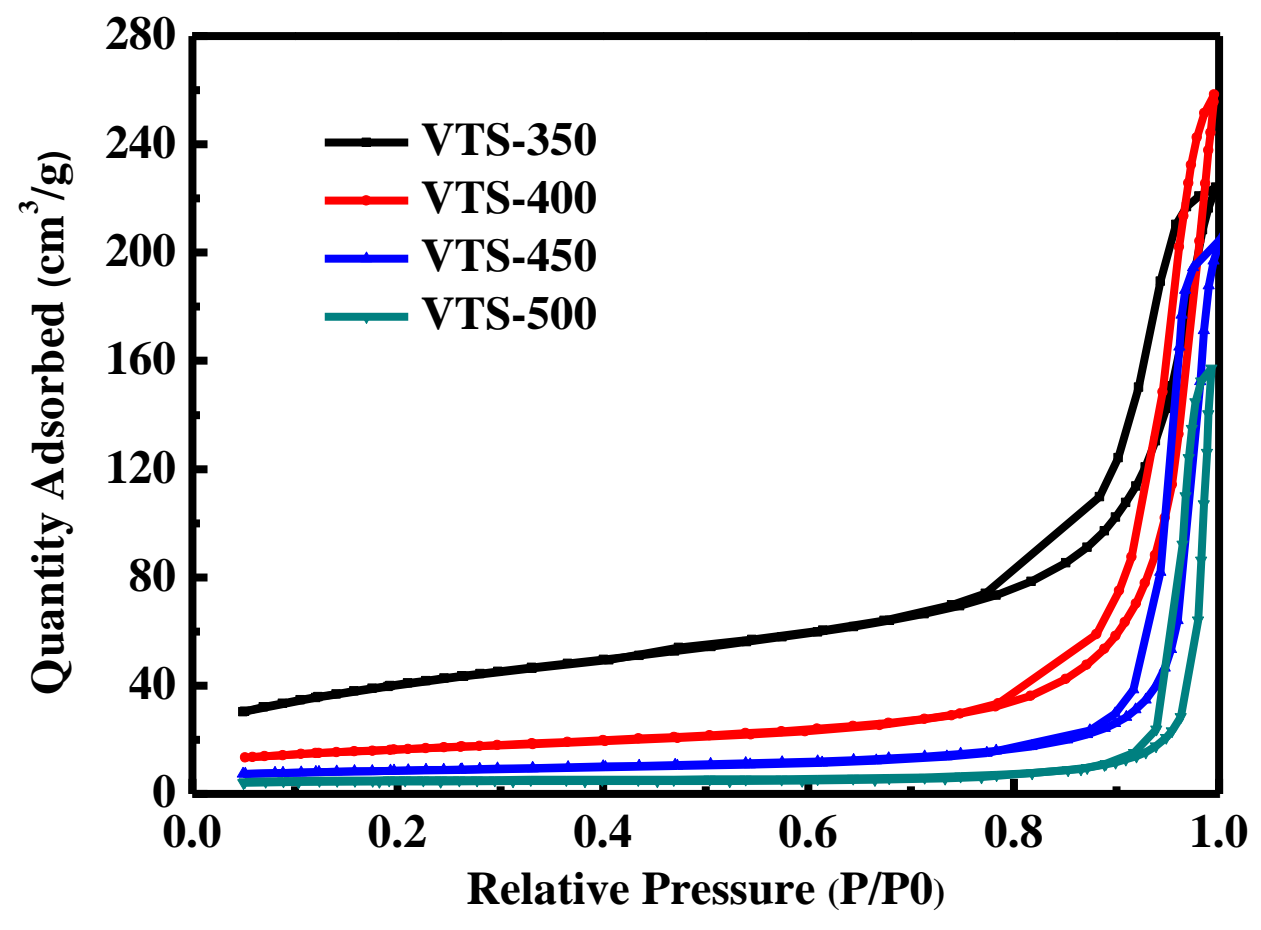


Figure S3. Potential Energy Surface $\left(\Delta \mathrm{E}_{0 \mathrm{~K}}, \mathrm{eV}\right)$ for the Formation of $\mathrm{HCHO}$ via the Alternative Pathway in the Hemiacetal Mechanism Calculated with Cluster Model (B3LYP).

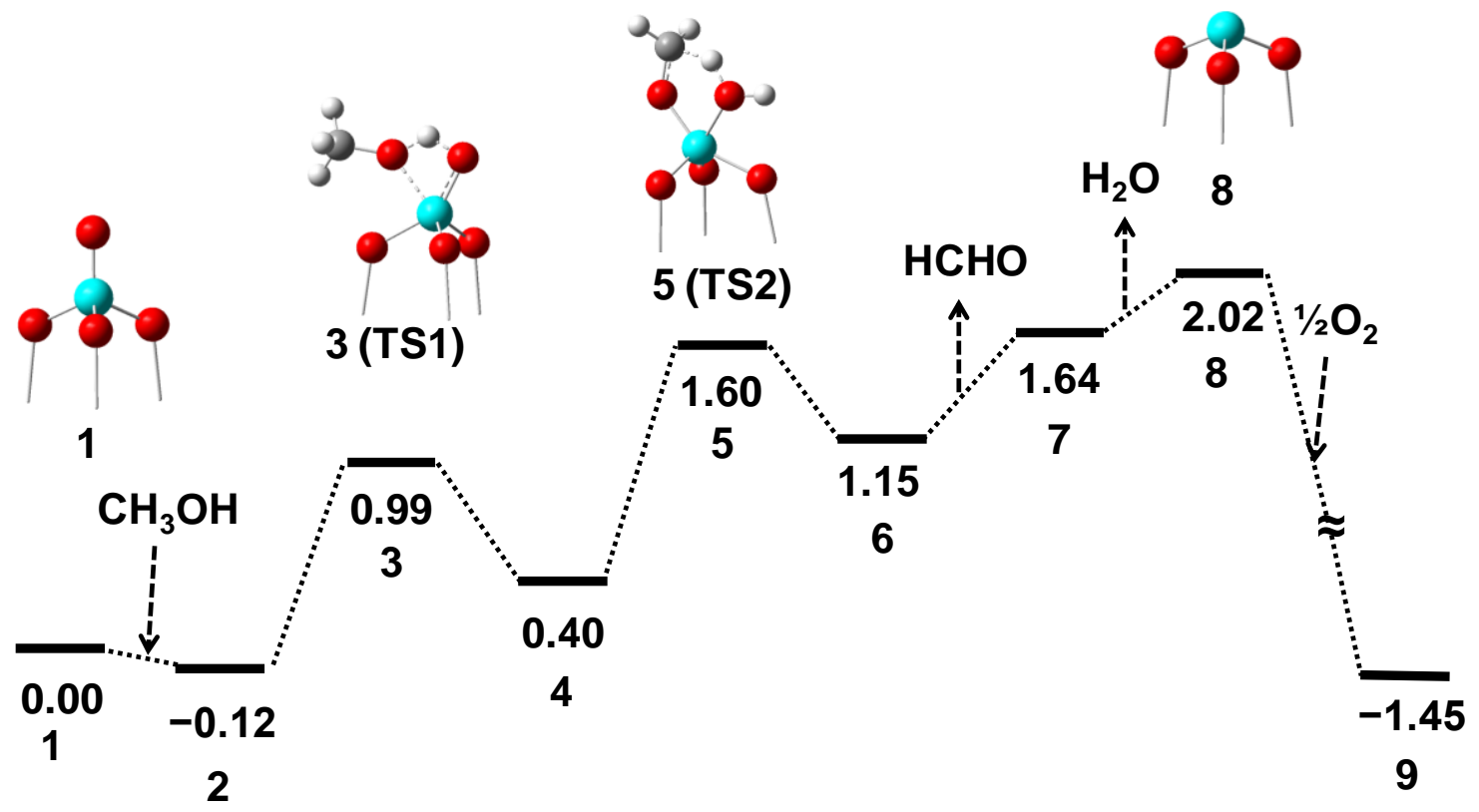

Figure S4. Potential Energy Surface $\left(\Delta \mathrm{E}_{0 \mathrm{~K}}, \mathrm{eV}\right)$ for the Formation of $\mathrm{HCOOCH}_{3}$ via the Alternative Pathway in the Hemiacetal Mechanism Calculated with Cluster Model (B3LYP).

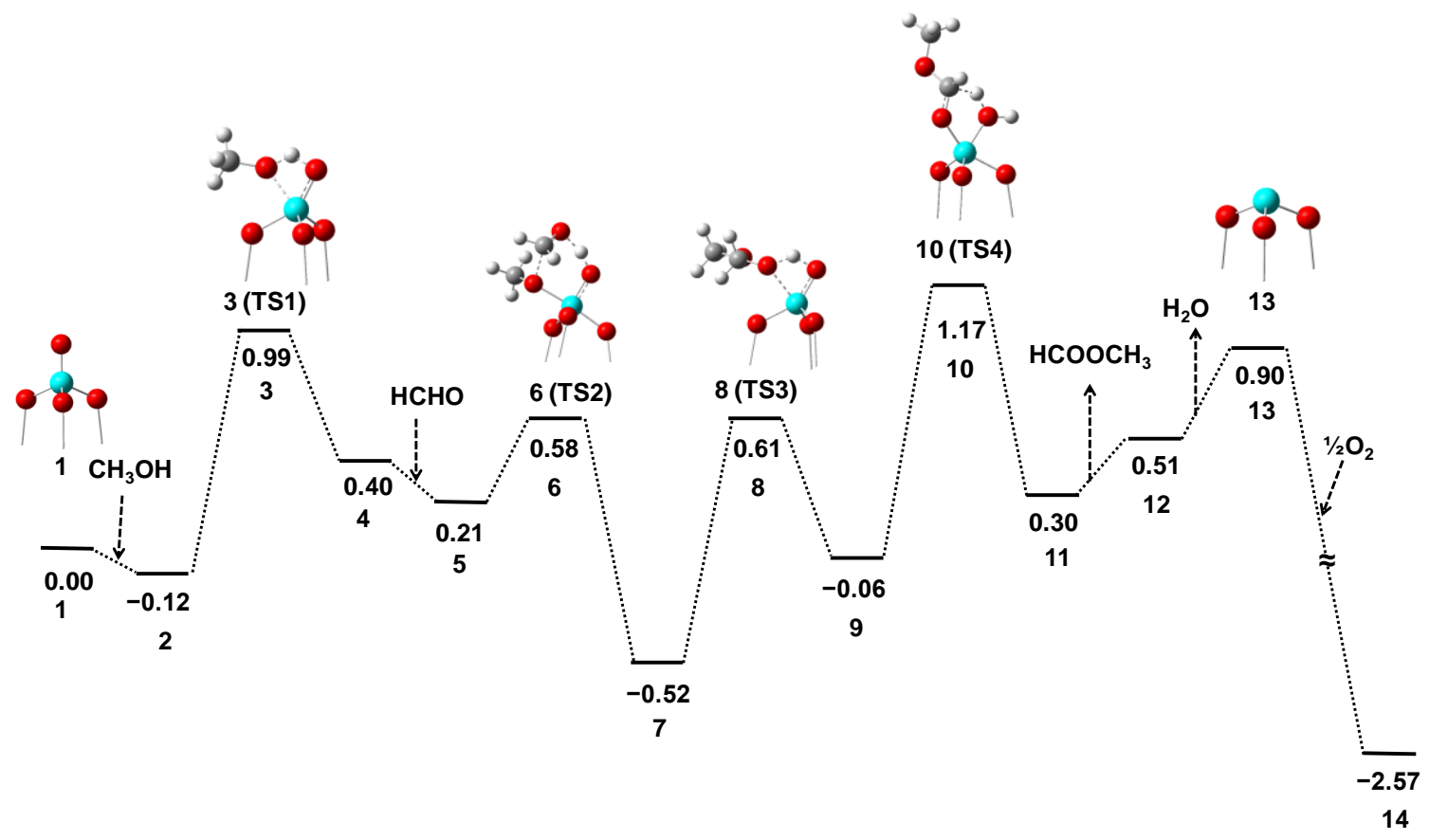


Figure S5. Structure of the Stationary States for the PES in Figures 2, 3, 4, S3 and S4.

For Figure 2: 1-11 (Periodic Slab)
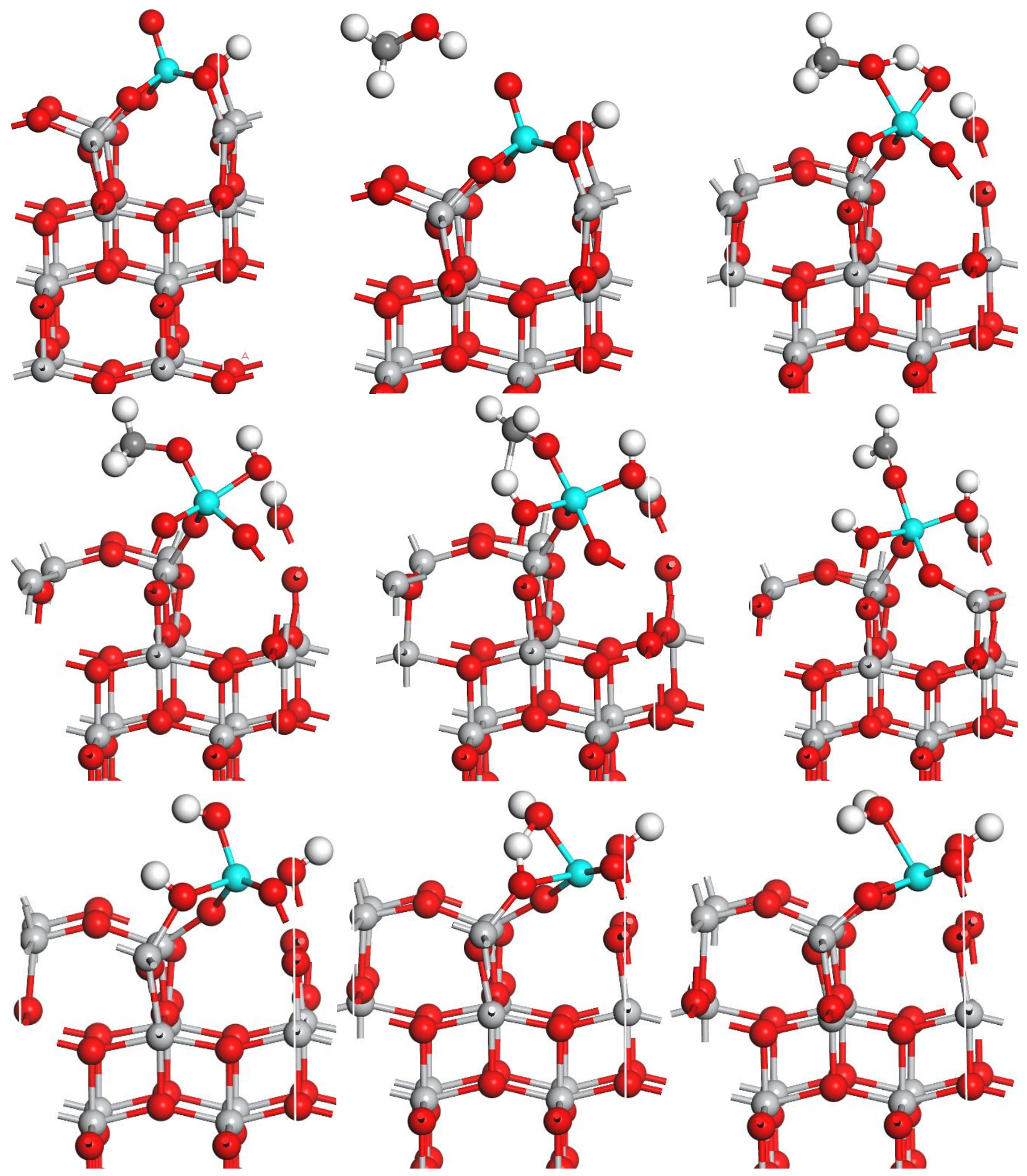


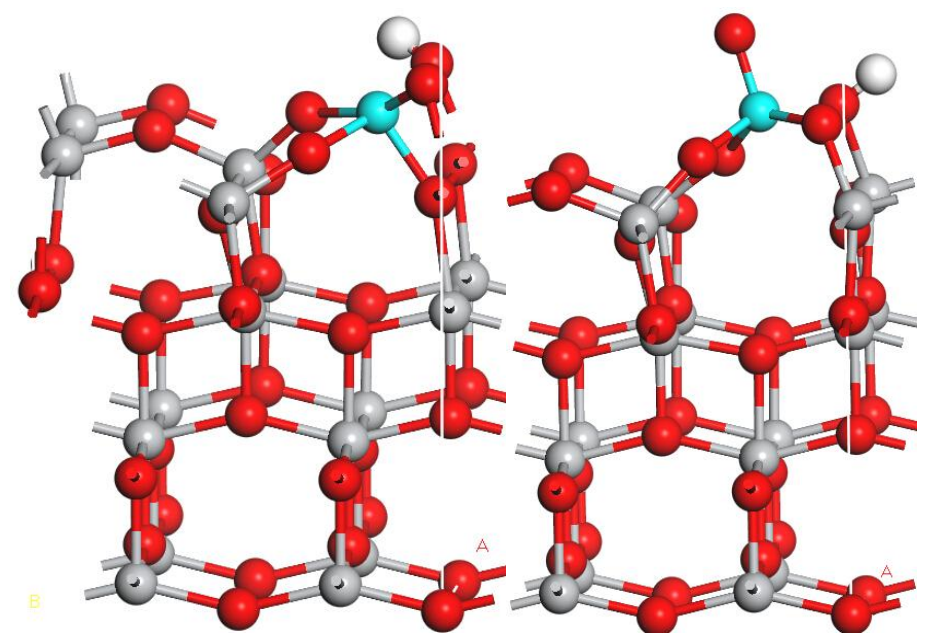


For Figure 2: 1-11 (Cluster)
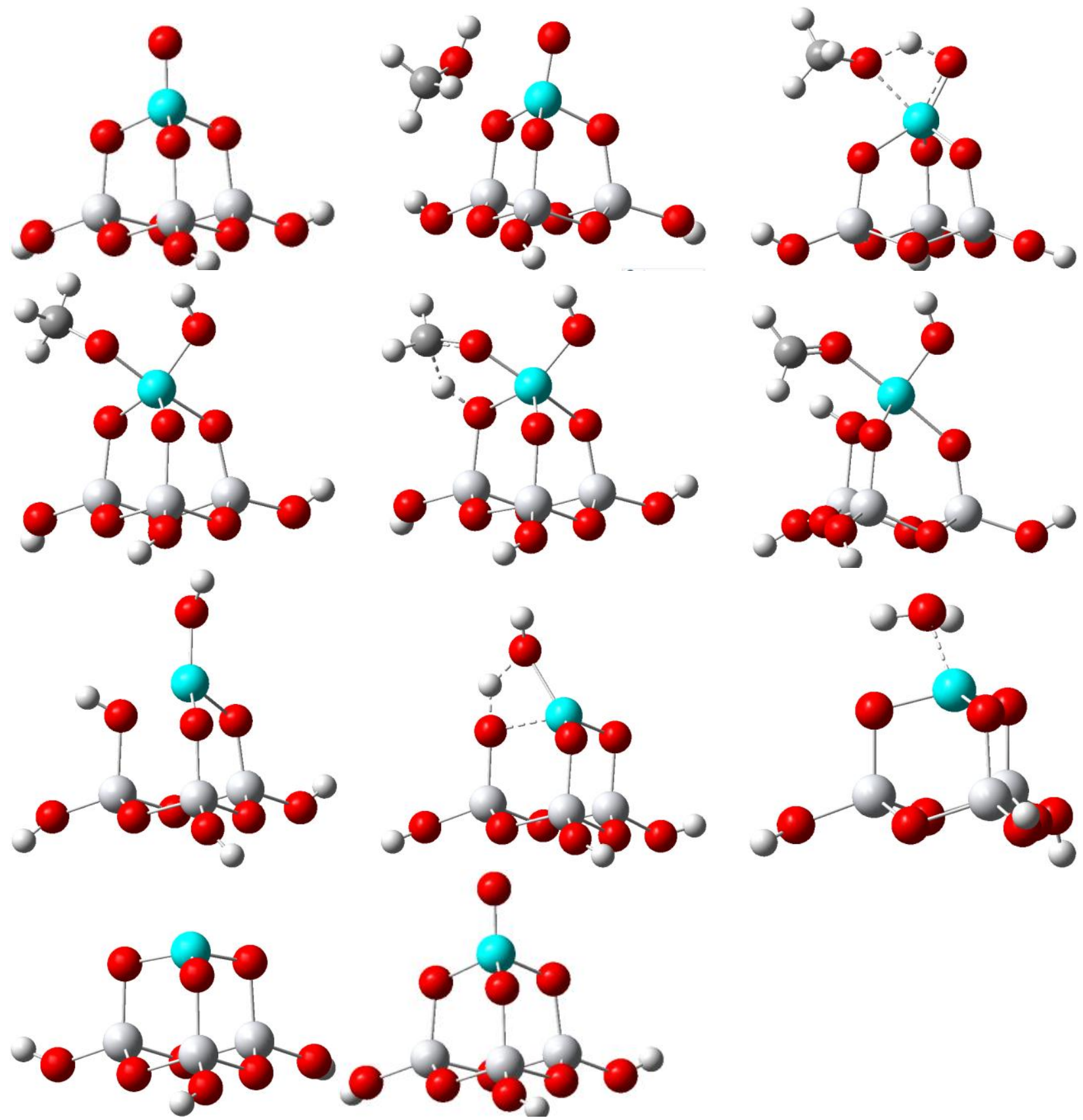
For Figure 3: 1-16 (Periodic Slab)

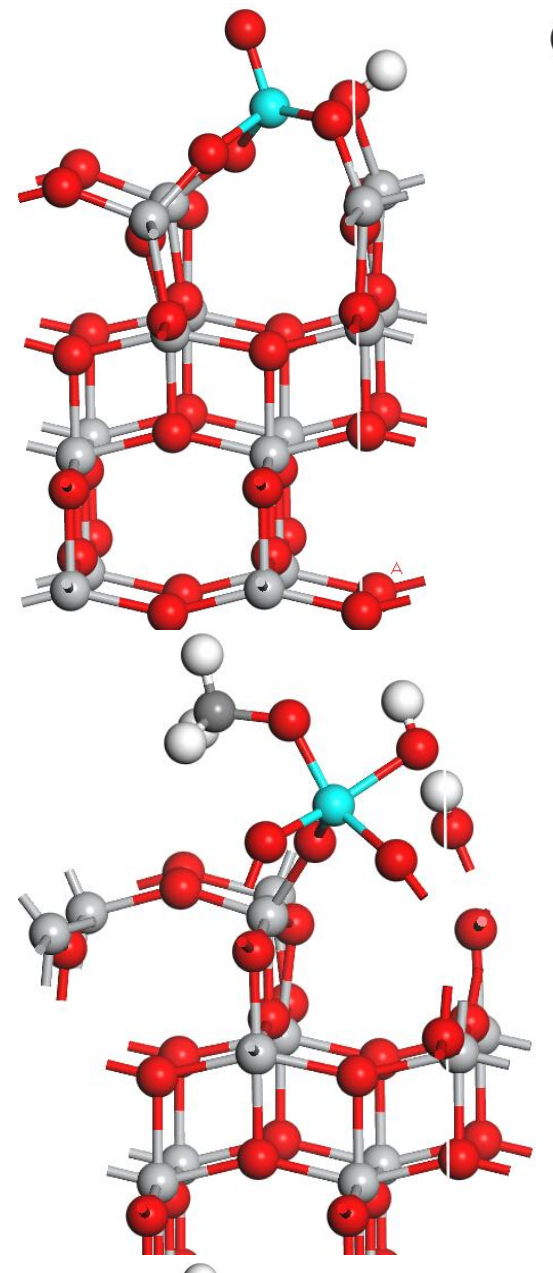

agdo

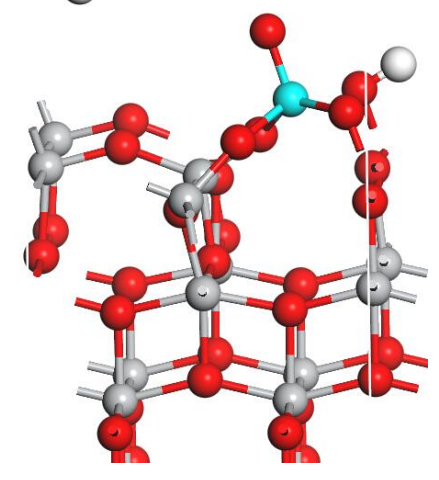

Q
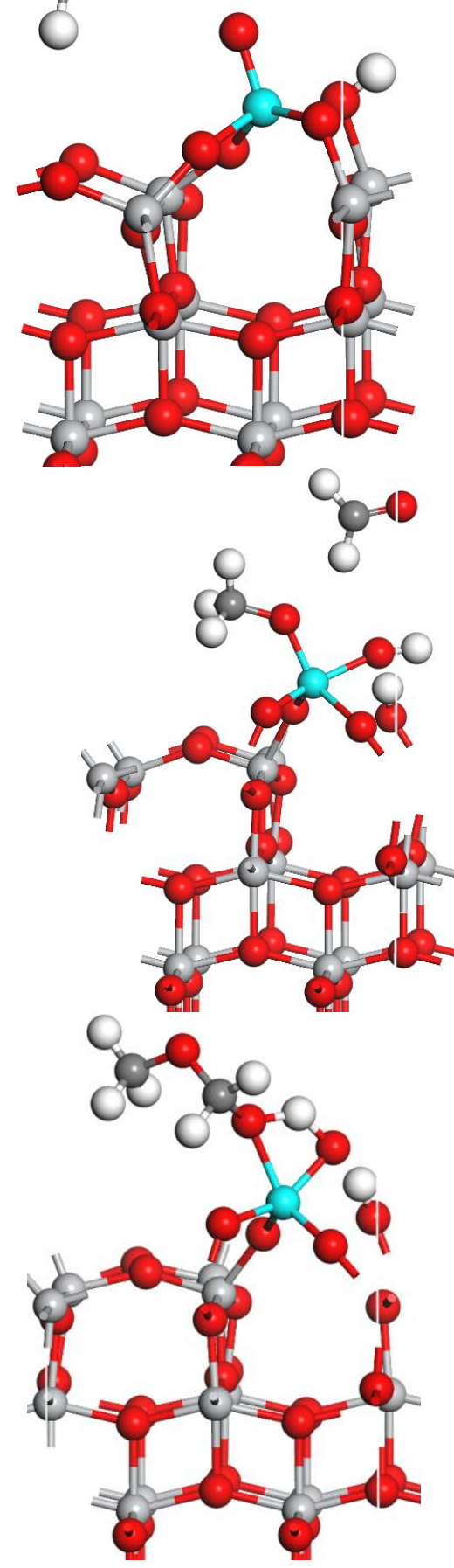
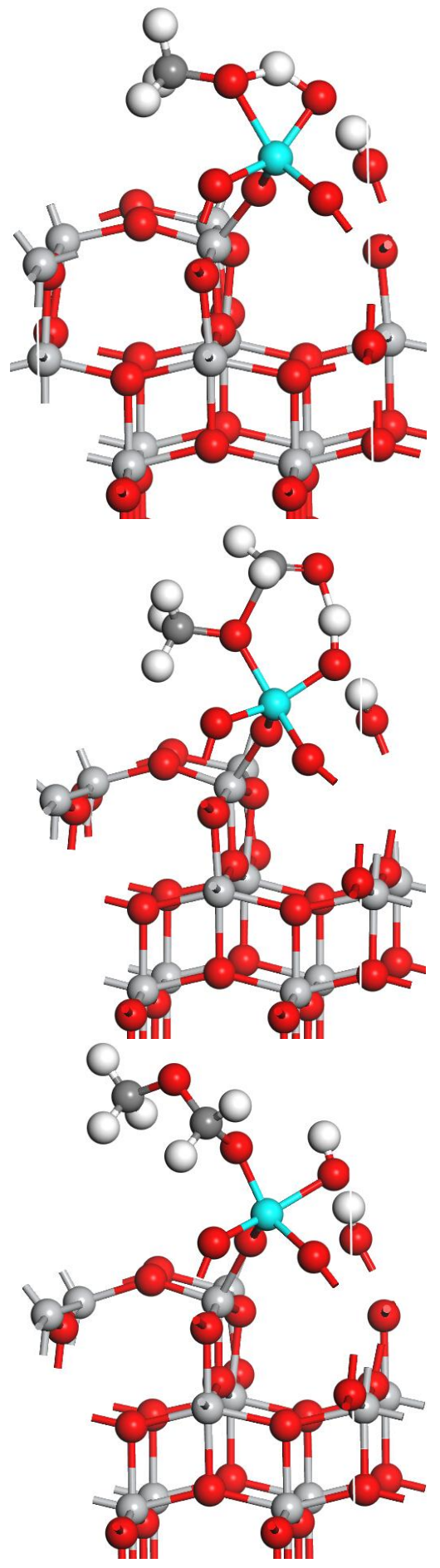

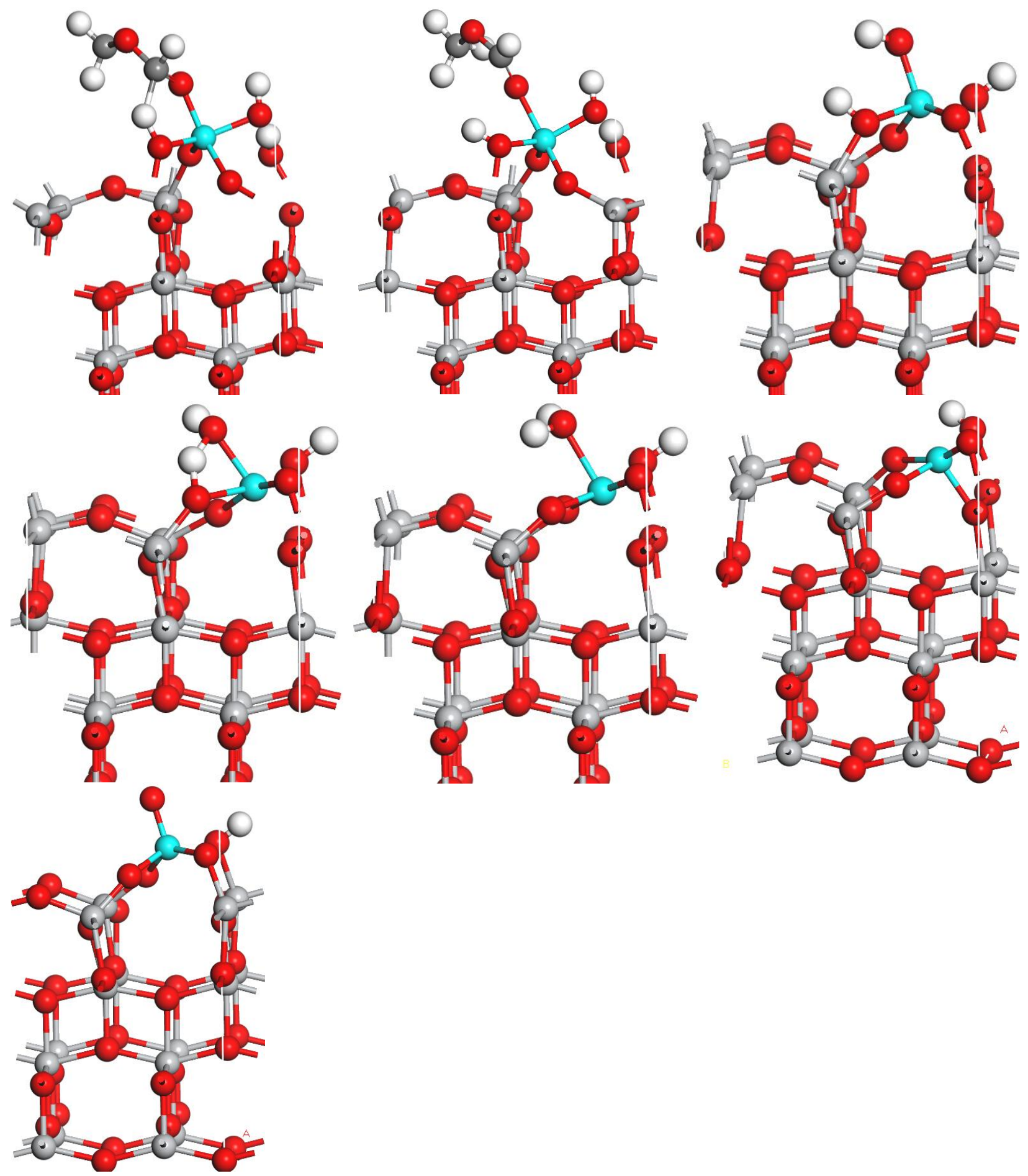
For Figure 3: 1-16 (Cluster)
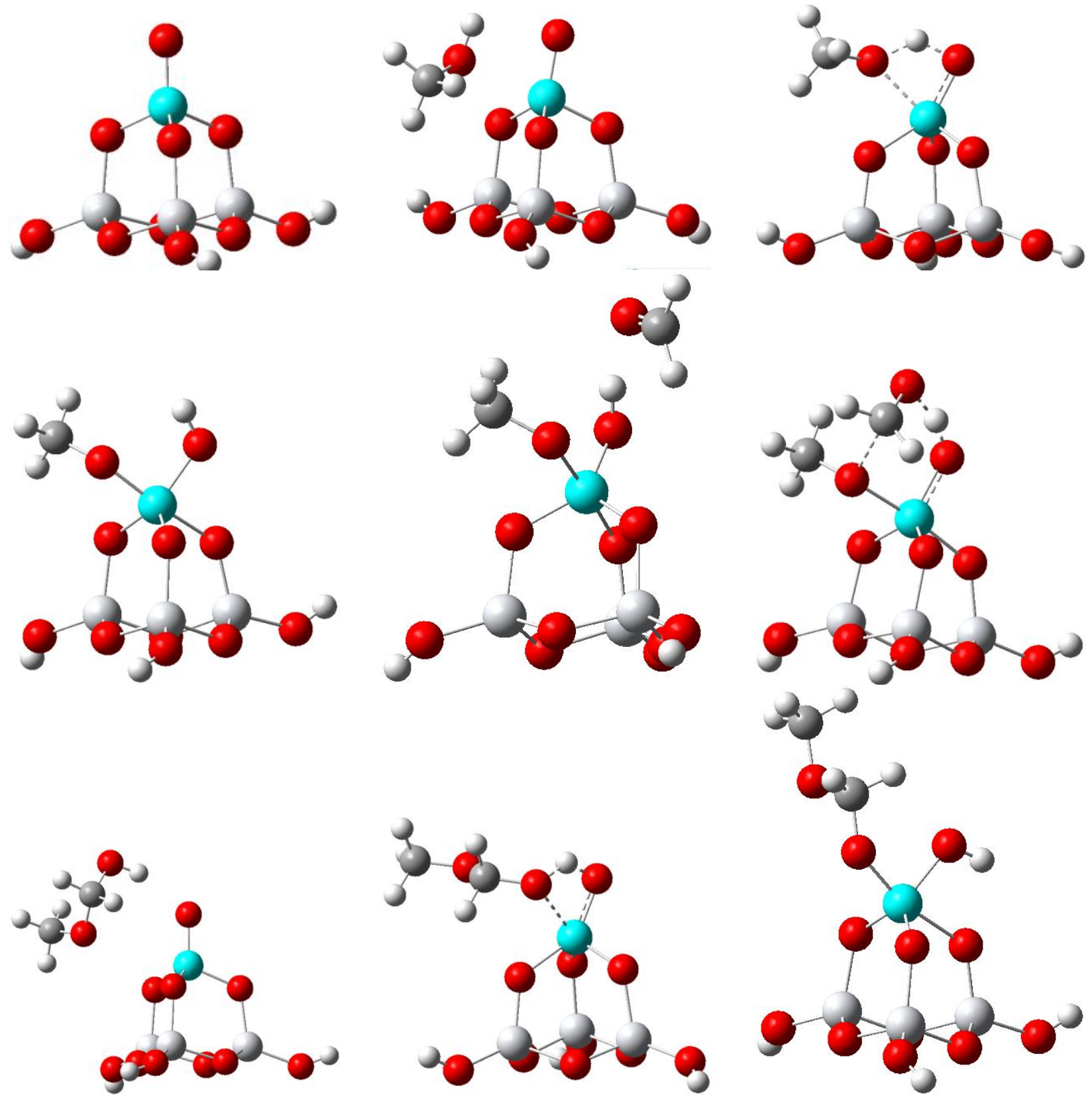


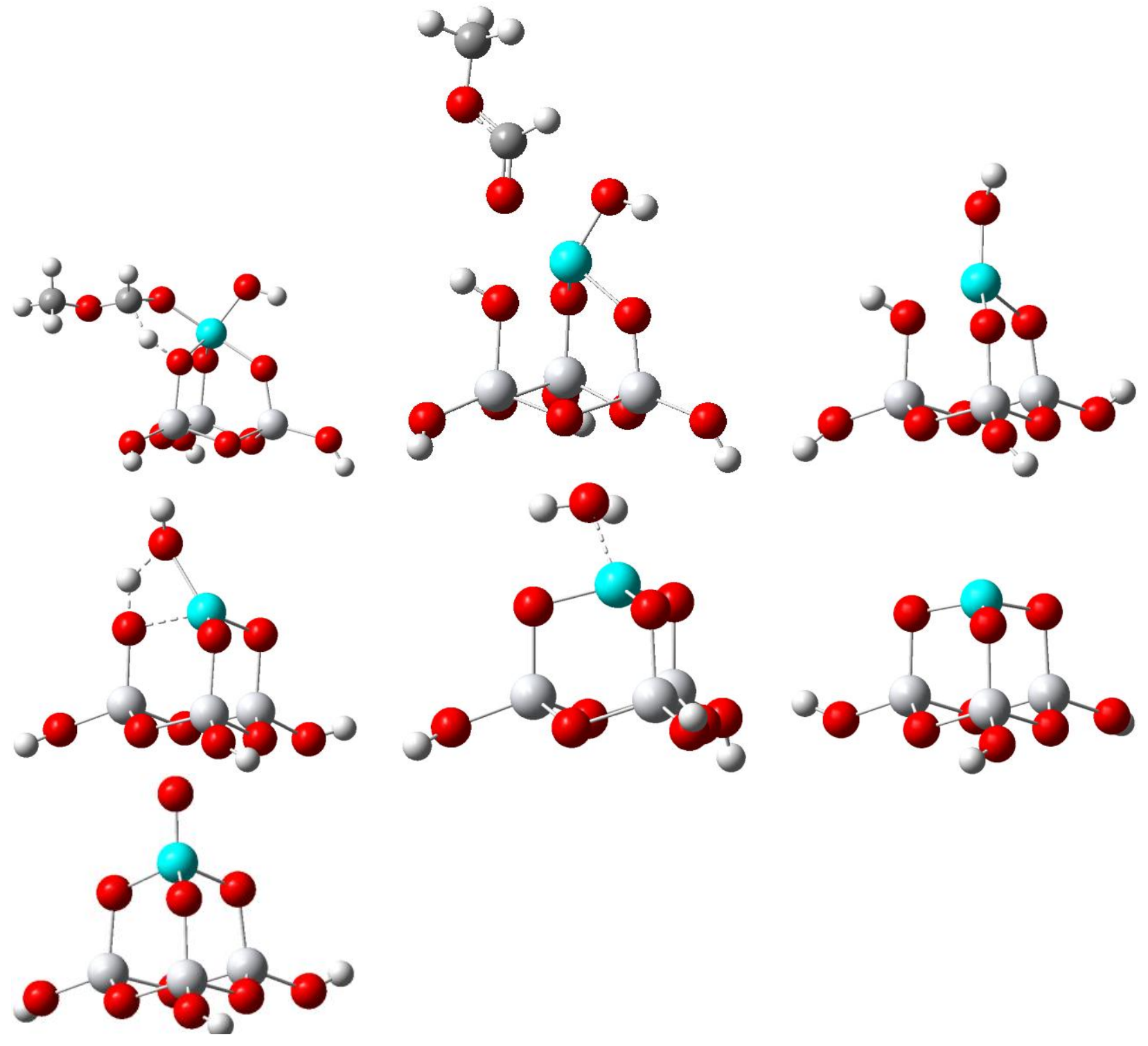


For Figure 4: 1-2a...-12 (Cluster)
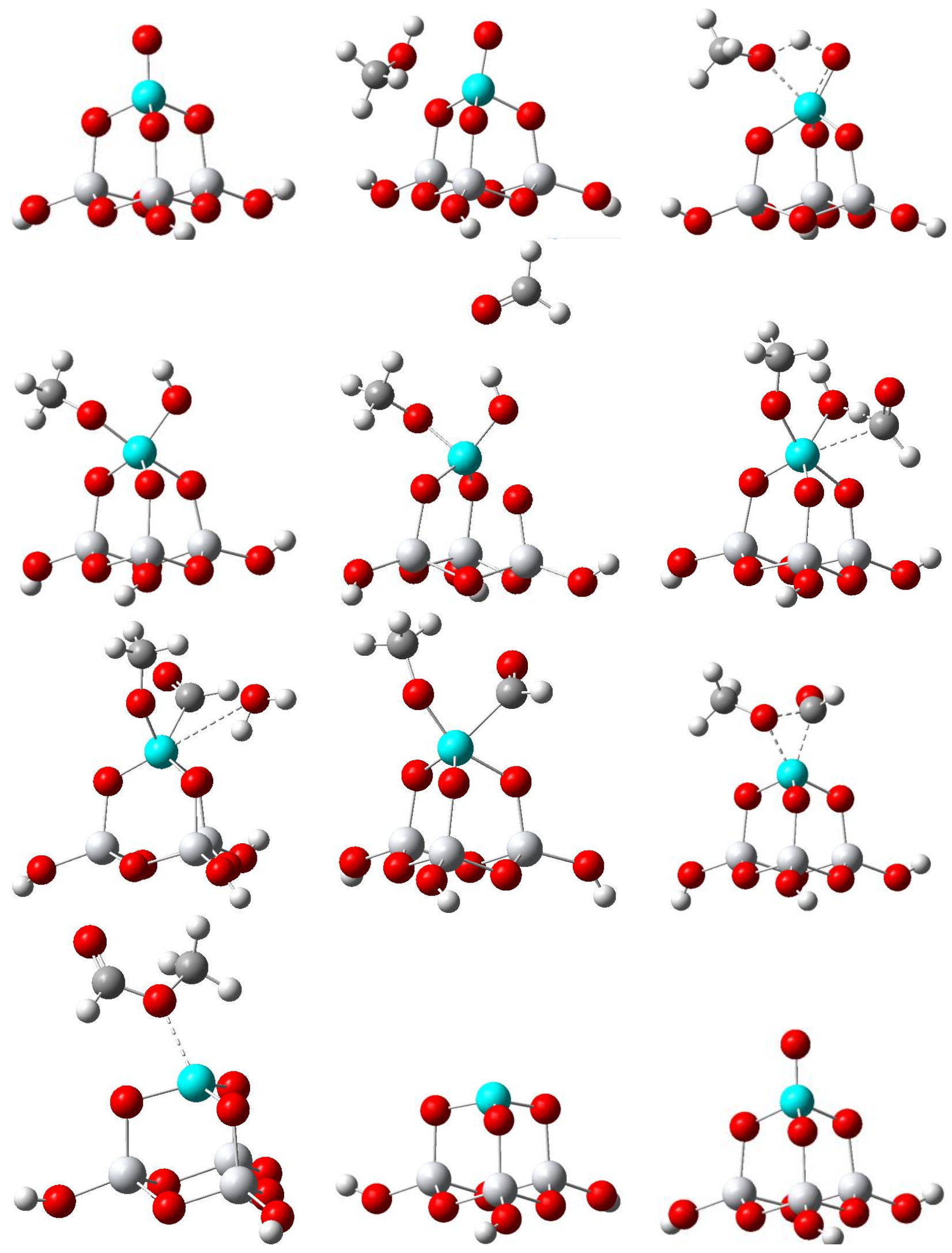
For Figure 4: 2b...6b (Cluster)
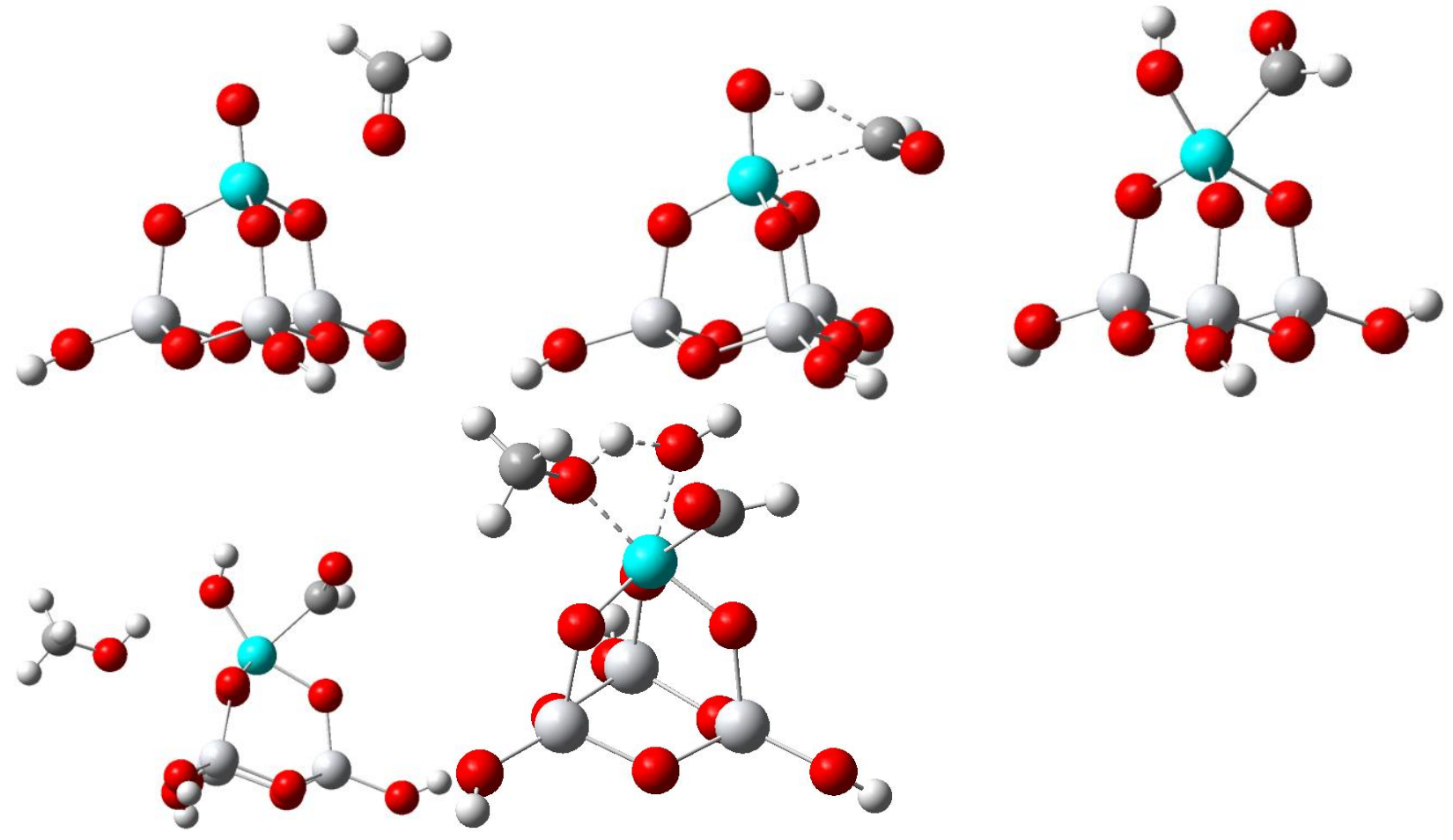
For Figure 6: 1-6 (Cluster).
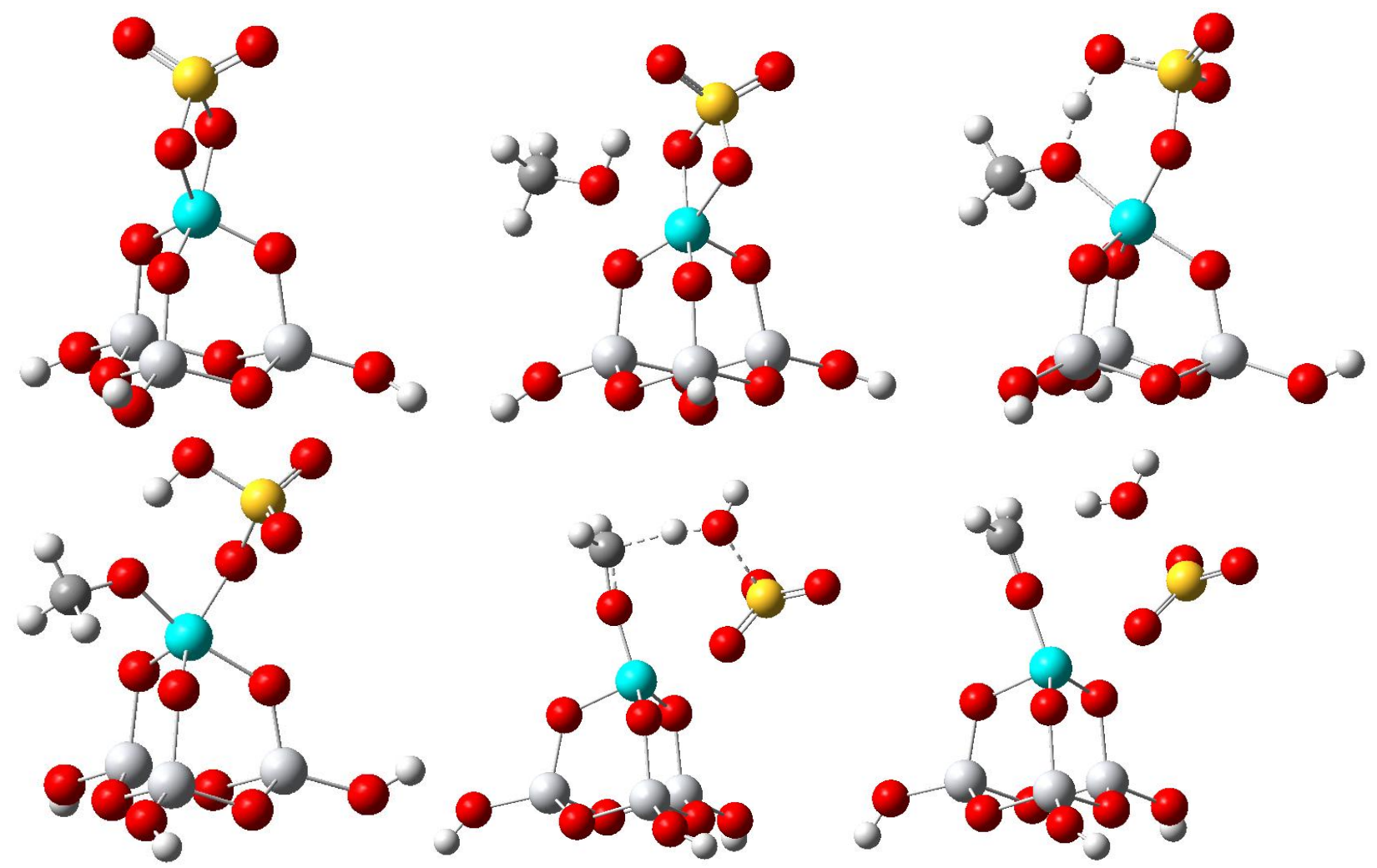
For Figure S3: 1-9 (Cluster)
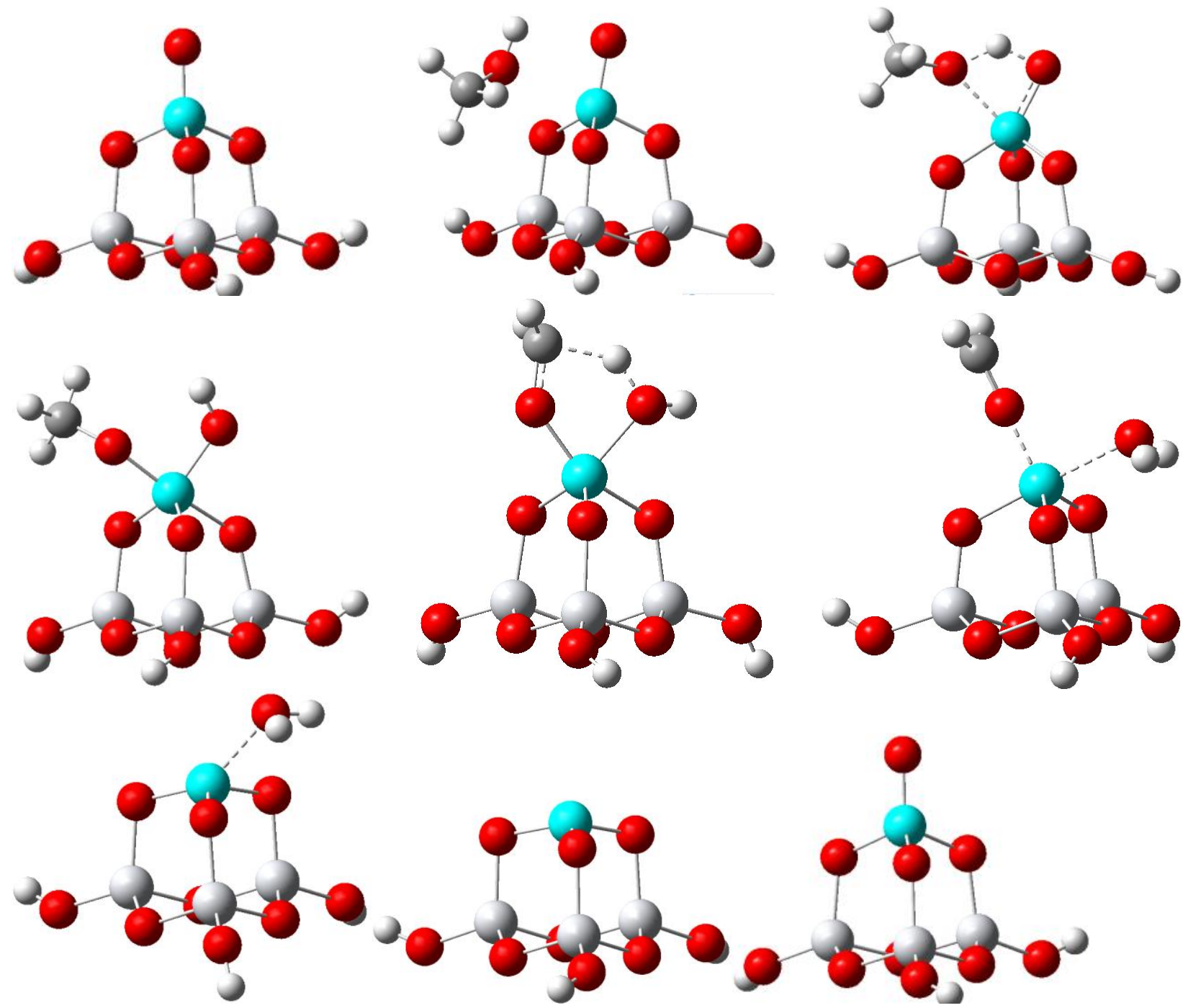
For Figure S4: 1-14 (Cluster)
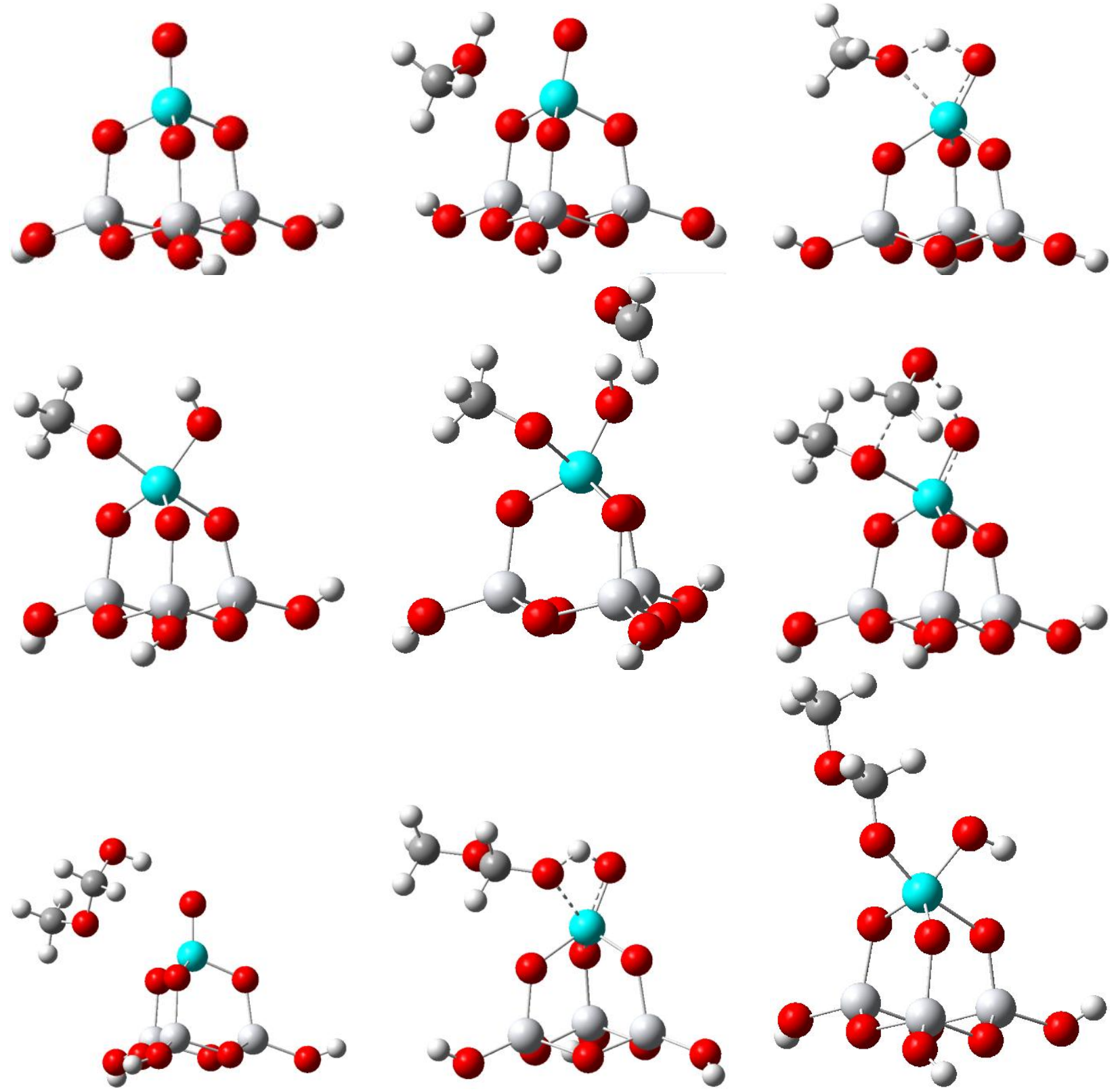

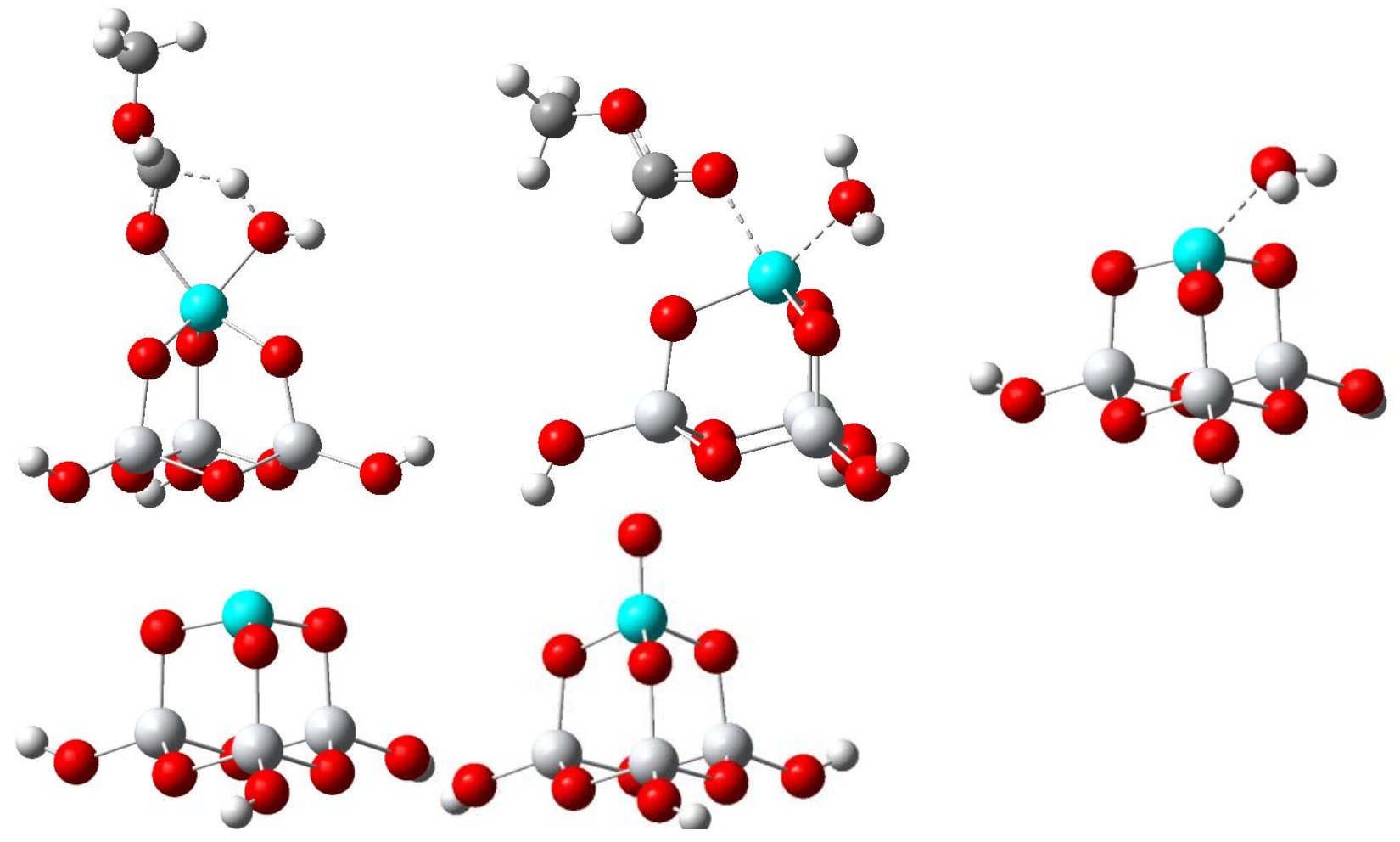
Table S1. Surface Areas (A), Pore Sizes and Pore Volumes Determined by $\mathrm{N}_{2}$ Adsorption for the VTS Catalysts.

\begin{tabular}{lccc}
\hline Catalyst & Surface Area $\left(\mathbf{m}^{\mathbf{2}} / \mathbf{g}\right)$ & Pore Size $(\mathbf{n m})$ & Pore Volume $\left(\mathbf{c m}^{\mathbf{3}} / \mathbf{g}\right)$ \\
\hline VTS-350 & 142 & 10 & 0.35 \\
VTS-400 & 55 & 29 & 0.40 \\
VTS-450 & 28 & 45 & 0.32 \\
VTS-500 & 16 & 61 & 0.24 \\
\hline
\end{tabular}

Table S2. V/Ti Atomic Ratios Determined by X-ray Photoelectron Spectroscopy and Sulfur Contents Measured by the Organic Elemental Analyzer for the VTS Catalysts.

\begin{tabular}{ccc}
\hline Catalyst & V/Ti Atomic Ratio & S (wt\%) \\
\hline VTS-350 & 0.36 & 1.86 \\
VTS-400 & 0.41 & 1.81 \\
VTS-450 & 0.30 & 1.66 \\
VTS-500 & 0.31 & 0.00 \\
\hline
\end{tabular}


Table S3. Reaction Temperatures $(\mathrm{T})$, Initial Total Pressure $\left(\mathrm{P}_{\text {total }}\right)$, Ratios of Partial Pressure $\left(\mathrm{P}_{\mathrm{O} 2} / \mathrm{P}_{\mathrm{CH} 3 \mathrm{OH}}\right.$ and $\left.\mathrm{P}_{\mathrm{N} 2} / \mathrm{P}_{\mathrm{O} 2}\right)$, and Molar Fractions $\left(\mathrm{Y}_{\mathrm{O} 2,0}\right.$ and $\left.\mathrm{Y}_{\mathrm{CH} 3 \mathrm{OH}, 0}\right)$, Methanol Conversion Rate $\left(\mathrm{X}_{\mathrm{CH} 3 \mathrm{OH}}\right)$, and Final Molar Fractions $\left(\mathrm{Y}_{\mathrm{MF}}, \mathrm{Y}_{\mathrm{DMM}}, \mathrm{Y}_{\mathrm{FA}}\right.$, and $\left.\mathrm{Y}_{\mathrm{DME}}\right)$. Reaction Conditions: $1 \mathrm{~g}$ of VTS-400 Catalyst, Liquid Hour Space Velocity (LHSV) of Methanol $1.521 \mathrm{~h}^{-1}$, Reaction Time $0.5 \mathrm{~h}$.

\begin{tabular}{|c|c|c|c|c|c|c|c|c|c|c|}
\hline $\begin{array}{c}\mathbf{T} \\
\left({ }^{\circ} \mathbf{C}\right)\end{array}$ & $\begin{array}{c}\mathbf{P}_{\text {total }} \\
\text { (MPa) }\end{array}$ & $\mathbf{P}_{\mathrm{O} 2} / \mathbf{P}_{\mathrm{CH} 3 \mathrm{OH}}$ & $\mathbf{P}_{\mathrm{N} 2} / \mathbf{P}_{\mathrm{O} 2}$ & $\begin{array}{c}Y_{\mathrm{CH3OH,0}} \\
(\%)\end{array}$ & $\begin{array}{c}Y_{\mathbf{O 2 , 0}} \\
(\%)\end{array}$ & $\begin{array}{c}\mathbf{X}_{\mathrm{CH3OH}} \\
(\%)\end{array}$ & $\mathbf{Y}_{\mathrm{MF}}(\%)$ & $\mathbf{Y}_{\text {DMM }}(\%)$ & $\mathbf{Y}_{\mathrm{FA}}(\%)$ & $Y_{\text {DME }}(\%)$ \\
\hline \multirow[t]{5}{*}{130} & 0.60 & 0.25 & 8.0 & 30.77 & 7.69 & 31.42 & 0.16 & 3.25 & 0.02 & 0.02 \\
\hline & 0.80 & 0.30 & 7.0 & 29.41 & 8.82 & 29.34 & 0.14 & 2.88 & 0.05 & 0.02 \\
\hline & 0.50 & 0.40 & 5.0 & 29.41 & 11.76 & 26.12 & 0.12 & 2.55 & 0.03 & 0.03 \\
\hline & 0.70 & 0.50 & 4.0 & 28.57 & 14.29 & 28.12 & 0.15 & 2.64 & 0.04 & 0.04 \\
\hline & 0.90 & 0.60 & 9.0 & 14.29 & 8.57 & 23.82 & 0.06 & 1.09 & 0.02 & 0.03 \\
\hline \multirow[t]{5}{*}{135} & 0.60 & 0.25 & 8.0 & 30.77 & 7.69 & 36.08 & 0.21 & 3.72 & 0.09 & 0.02 \\
\hline & 0.80 & 0.30 & 7.0 & 29.41 & 8.82 & 39.23 & 0.24 & 3.85 & 0.11 & 0.03 \\
\hline & 0.50 & 0.40 & 5.0 & 29.41 & 11.76 & 33.44 & 0.20 & 3.26 & 0.07 & 0.04 \\
\hline & 0.70 & 0.50 & 4.0 & 28.57 & 14.29 & 34.76 & 0.21 & 3.27 & 0.07 & 0.06 \\
\hline & 0.90 & 0.60 & 9.0 & 14.29 & 8.57 & 29.23 & 0.09 & 1.32 & 0.03 & 0.04 \\
\hline \multirow[t]{5}{*}{140} & 0.60 & 0.25 & 8.0 & 30.77 & 7.69 & 44.65 & 0.38 & 4.55 & 0.18 & 0.03 \\
\hline & 0.80 & 0.30 & 7.0 & 29.41 & 8.82 & 46.10 & 0.40 & 4.46 & 0.18 & 0.05 \\
\hline & 0.50 & 0.40 & 5.0 & 29.41 & 11.76 & 41.41 & 0.33 & 4.00 & 0.13 & 0.05 \\
\hline & 0.70 & 0.50 & 4.0 & 28.57 & 14.29 & 41.57 & 0.33 & 3.87 & 0.12 & 0.09 \\
\hline & 0.90 & 0.60 & 9.0 & 14.29 & 8.57 & 37.84 & 0.17 & 1.68 & 0.07 & 0.06 \\
\hline \multirow[t]{2}{*}{145} & 0.60 & 0.25 & 8.0 & 30.77 & 7.69 & 53.14 & 0.73 & 5.25 & 0.32 & 0.04 \\
\hline & 0.80 & 0.30 & 7.0 & 29.41 & 8.82 & 54.74 & 0.81 & 5.08 & 0.32 & 0.07 \\
\hline
\end{tabular}




\begin{tabular}{|l|l|l|l|l|l|l|l|l|l|l|}
\hline & 0.50 & 0.40 & 5.0 & 29.41 & 11.76 & 49.31 & 0.60 & 4.64 & 0.24 & 0.07 \\
\hline & 0.70 & 0.50 & 4.0 & 28.57 & 14.29 & 50.61 & 0.74 & 4.52 & 0.23 & 0.15 \\
\hline & 0.90 & 0.60 & 9.0 & 14.29 & 8.57 & 43.97 & 0.25 & 1.91 & 0.10 & 0.07 \\
\hline $\mathbf{1 5 0}$ & 0.60 & 0.25 & 8.0 & 30.77 & 7.69 & 60.19 & 1.36 & 5.56 & 0.52 & 0.06 \\
\hline & 0.80 & 0.30 & 7.0 & 29.41 & 8.82 & 62.59 & 1.66 & 5.26 & 0.54 & 0.08 \\
\hline & 0.50 & 0.40 & 5.0 & 29.41 & 11.76 & 55.34 & 0.92 & 5.03 & 0.33 & 0.11 \\
\hline & 0.70 & 0.50 & 4.0 & 28.57 & 14.29 & 59.11 & 1.32 & 4.89 & 0.40 & 0.19 \\
\hline & 0.90 & 0.60 & 9.0 & 14.29 & 8.57 & 52.36 & 0.45 & 2.15 & 0.18 & 0.09 \\
\hline
\end{tabular}


Table S4. Supercell Parameters $(\AA)$ and Fractional Coordinates of the Periodic Slab Model of the $\mathrm{V}_{2} \mathrm{O}_{5} / \mathrm{TiO}_{2}$ Catalysts.

\begin{tabular}{|c|c|c|c|c|c|c|c|}
\hline \multicolumn{2}{|c|}{7.6100000000000003} & \multicolumn{2}{|c|}{0.0000000000000000} & \multicolumn{3}{|c|}{0.0000000000000000} & \\
\hline 0.00000000000000 & 00 & 0.00000000000 & 0000 & 23.20799999 & & & \\
\hline 0 & $\mathrm{Ti}$ & & & & & & \\
\hline 35 & 16 & & & & & & \\
\hline 0.4051038130216208 & -0.0 & 30317702429509 & 0.442 & 91199524813 & $\mathbf{T}$ & $\mathbf{T}$ & $\mathbf{T}$ \\
\hline 0.2364717762334615 & 0.23 & 357493268927737 & 0.4172 & 2590966879396 & $\mathbf{T}$ & $\mathbf{T}$ & $\mathbf{T}$ \\
\hline 0.0000000000000000 & 0.0 & 00000000000000 & 0.000 & 0000000000000 & $\mathbf{F}$ & $\mathbf{F}$ & $\mathbf{F}$ \\
\hline 0.2486143703036373 & 0.2 & 79193283345613 & 0.212 & 1780990694896 & $\mathbf{T}$ & $\mathbf{T}$ & $\pi$ \\
\hline 0.0000000000000000 & 0.25 & 500657030223365 & $0.104^{\circ}$ & 7483626335719 & & $\mathbf{F}$ & \\
\hline 0.2511603945749757 & 0.0 & 81196358509390 & 0.316 & 5291049817612 & $\mathbf{T}$ & $\mathbf{T}$ & \\
\hline 0.2500657030223365 & 0.0 & 00000000000000 & 0.144 & 1744226128918 & $\mathbf{F}$ & $\mathbf{F}$ & $\mathbf{F}$ \\
\hline 0.0706588622530305 & 0.38 & 322503705102002 & 0.384 & 1501603406702 & $\mathbf{T}$ & $\mathbf{T}$ & $\mathbf{T}$ \\
\hline 0.2500657030223365 & 0.25 & 500657030223365 & 0.039 & 4260599793199 & $\mathbf{F}$ & $\mathbf{F}$ & $\mathbf{F}$ \\
\hline .0040492885030468 & $-0.0 c$ & 52167695876586 & 0.252 & 5593402644126 & $\mathbf{T}$ & $\mathbf{T}$ & \\
\hline 0.5000000000000000 & & 000000000000000 & 0.000 & 0000000000000 & & $\mathbf{F}$ & \\
\hline 0.7483686756572577 & $0.2-2+x-1$ & 69651370326590 & 0.2 & 5191571 & $\mathbf{T}$ & $\mathbf{T}$ & \\
\hline 0.5000000000000000 & 0. & 500657030223365 & $0.104^{\circ}$ & 7483626335719 & $\mathbf{F}$ & $\mathbf{F}$ & \\
\hline 0.7490079252109135 & 0.03 & 320938638441808 & 0.324 & 1901736009581 & $\mathbf{T}$ & $\mathbf{T}$ & $\mathbf{T}$ \\
\hline 0.7500657030223365 & 0.0 & 0000 & 0.144 & 1744 & $F$ & $\mathbf{F}$ & \\
\hline 0.4236577565360100 & 0.3 & $1092^{\circ}$ & 0.3742 & 2617 & & $\mathbf{T}$ & \\
\hline 0.7500657030223365 & 0.2 & 000570302 & 0.039 & 4260 & & $\mathbf{F}$ & \\
\hline 0.5038465370789978 & -0.0 & 96080500984819 & 0.2 & 5367 & $\mathbf{T}$ & $\mathbf{T}$ & \\
\hline 0.0000000000000000 & 0.5 & 00000000000000 & 0.000 & 000 & $\mathbf{F}$ & $\mathbf{F}$ & $\mathbf{F}$ \\
\hline 0.2477639704373805 & 0.75 & 29695357187938 & 0.2098 & 8934135670781 & $\mathbf{T}$ & $\mathbf{T}$ & \\
\hline 0.0000000000000000 & 0.75 & 5006 & 0.1 & & & $\mathbf{F}$ & \\
\hline 0.2481824921372816 & 0.5 & $220 \varepsilon$ & 0.308 & 999 & & $\mathbf{T}$ & \\
\hline 0.2500657030223365 & 0.5 & 000000 & 0.1 & 1744 & $F$ & $\mathbf{F}$ & \\
\hline-0.0236564727121706 & 0.75 & 35066518971251 & 0.359 & 9033994126737 & $\mathbf{T}$ & $\mathbf{T}$ & $T$ \\
\hline 0.2500657030223365 & 0.75 & 000657030223365 & 0.039 & 42605997 & $\mathbf{F}$ & $\mathbf{F}$ & 2 \\
\hline-0.0024381326804748 & 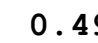 & $027^{\prime}$ & & & $\mathbf{T}$ & $\mathbf{T}$ & \\
\hline 10000000000 & 0.5 & 000 & 0.0 & & 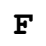 & $\mathbf{F}$ & \\
\hline 0.7495514652860051 & 0.7 & 92255520543537 & 0.212 & 3719031234022 & & $\mathbf{T}$ & $\mathbf{T}$ \\
\hline 0.5000000000000000 & 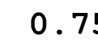 & 00657030223365 & $0.104^{\circ}$ & 7483626335719 & $\mathbf{F}$ & $\mathbf{F}$ & $\mathbf{F}$ \\
\hline 0.7479843036068982 & $0.4 \mathrm{~S}-2.50$ & 05964080626816 & 0.316 & 1342384173855 & $\mathbf{T}$ & $\mathbf{T}$ & $\mathbf{T}$ \\
\hline 0.7500657030223365 & 0.5 & 00000000000000 & 0.144 & 1744226128918 & $\mathbf{F}$ & $\mathbf{F}$ & 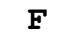 \\
\hline 0.5178240794050780 & 0.7 & 98688412113128 & 0.357 & & $T$ & $\mathbf{T}$ & \\
\hline 0.7500657030223365 & 0.75 & 500657030223365 & 0.0 & & & $\mathbf{F}$ & \\
\hline 0.4987860380 & $0.4 \xi$ & 3825 & 0.2 & & & $\mathbf{T}$ & \\
\hline 0.0729287237990261 & 0.0 & 525027540901179 & 0.411 & 5484043543388 & $\mathbf{T}$ & $\mathbf{T}$ & $\mathbf{T}$ \\
\hline 0.2462217176809563 & 0.25 & 65965942903993 & $0.482^{\circ}$ & 7466254614906 & $\mathbf{T}$ & $\mathbf{T}$ & $\mathbf{T}$ \\
\hline 0.4297594849592817 & 0.0 & 53910501085832 & 0.4168 & 8936524393111 & $\mathbf{T}$ & $\mathbf{T}$ & $\mathbf{T}$ \\
\hline-0.0015610869792806 & -0.02 & & & & $\mathbf{T}$ & $\mathbf{T}$ & \\
\hline 0.2500657030223365 & 0.25 & 500657030223365 & 0.124 & & $\mathbf{F}$ & $\mathbf{F}$ & \\
\hline 0.0000000000000000 & & 000657030223365 & $0.019^{\circ}$ & 734 & $\mathbf{F}$ & $\mathbf{F}$ & \\
\hline 0.2472911012726211 & 0.0 & 09057767014220 & 0.2322 & 2187704998555 & $\mathbf{T}$ & $\mathbf{T}$ & $\mathbf{T}$ \\
\hline 0.5049544399644044 & -0.02 & 64150442533282 & 0.338 & 6036304526032 & $\mathbf{T}$ & $\mathbf{T}$ & $\mathbf{T}$ \\
\hline 0.7500657030223365 & 0.25 & 000657030223365 & 0.124 & 4398483281657 & $\mathbf{F}$ & $\mathbf{F}$ & \\
\hline 0.5000000000000000 & & 500657030223365 & 0.019 & 7345 & 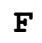 & $\mathbf{F}$ & \\
\hline 0.7507124626602042 & 0. & 14470255808333 & 0.2312 & 05047 & $\mathbf{T}$ & $\mathbf{T}$ & \\
\hline 0.0000263743196436 & & 327436087778121 & $0.330^{\circ}$ & 7630126412798 & $\mathbf{T}$ & $\mathbf{T}$ & \\
\hline 0.2500657030223365 & 0.75 & 00657030223365 & 0.124 & 4398483281657 & $\mathbf{F}$ & & \\
\hline
\end{tabular}


0.0000000000000000

0.2465328740490558

0.4943866350344449

0.7500657030223365

0.5000000000000000

0.7488350124620003
0.7500657030223365

0.5020187215540833

0.5194676308167514

0.7500657030223365

0.7500657030223365

0.4970327202626066

$\begin{array}{llll}0.0197345742847261 & F & F & F \\ 0.2284318567708238 & T & T & T \\ 0.3297756058434959 & T & T & T \\ 0.1244398483281657 & F & F & F \\ 0.0197345742847261 & F & F & F \\ 0.2294002681317916 & T & T & T\end{array}$

Table S5. Cartesian Coordinates $(\AA)$ of the Cluster Model of the $\mathrm{V}_{2} \mathrm{O}_{5} / \mathrm{TiO}_{2}$ Catalysts.

$\begin{array}{lrrr}0 & -0.885132 & -1.165285 & 1.416844 \\ \mathrm{O} & -1.584561 & 1.231879 & 0.000000 \\ \mathrm{O} & 0.883324 & 1.068079 & 1.438941 \\ \mathrm{O} & -0.885132 & -1.165285 & -1.416844 \\ \mathrm{O} & 1.567503 & -1.298296 & 0.000000 \\ \mathrm{O} & -1.735127 & 1.312092 & -3.053427 \\ \mathrm{O} & 0.883324 & 1.068079 & -1.438941 \\ \mathrm{O} & 3.596272 & 1.032177 & 0.000000 \\ \mathrm{~V} & -0.122387 & -1.912423 & 0.000000 \\ \mathrm{O} & -1.735127 & 1.312092 & 3.053427 \\ \mathrm{O} & -0.217413 & -3.485584 & 0.000000 \\ \mathrm{TI} & -0.885132 & 0.679062 & -1.594703 \\ \mathrm{TI} & 1.874420 & 0.498470 & 0.000000 \\ \mathrm{TI} & -0.885132 & 0.679062 & 1.594703 \\ \mathrm{H} & 4.476163 & 0.643005 & 0.000000 \\ \mathrm{H} & -1.528074 & 1.609049 & -3.944644 \\ \mathrm{H} & -1.528074 & 1.609049 & 3.944644\end{array}$

Table S6. Fractional Coordinates of Stationary States Calculated with the Periodic Slab Model.

The Same Supercell Parameters Are Identical to Those Given in Table S4.

For Figure 2: 2-10

2

$\begin{array}{lllll}\mathrm{H} & \mathrm{C} & \mathrm{V} & \mathrm{O} & \mathrm{Ti}\end{array}$

$\begin{array}{lllll}5 & 1 & 1 & 36 & 16\end{array}$

$0.4021650417925065-0.0555029365234857$

$\begin{array}{lll}0.2053136526824790 & 0.8723790692988502\end{array}$

$\begin{array}{lll}0.1950218055726342 & 0.7747409744182425\end{array}$

0.3949485445177621

0.2167545684172112

0.7627847514507866

0.5026976871610860

0.2494191639992666

0.7593796805949922

0.2375688565403317

0.2350468109027780

0.0000000000000000

0.0000000000000000

0.2490834818351992

0.2480040087209304

0.0000000000000000

0.2500657030223365

0.2510269548929346

0.0061705618490033

0.2500657030223365

0.0000000000000000

$\begin{array}{llll}0.4426074853221137 & \mathrm{~T} & \mathrm{~T} & \mathrm{~T} \\ 0.5547977657687174 & \mathrm{~T} & \mathrm{~T} & \mathrm{~T} \\ 0.4851370962237689 & \mathrm{~T} & \mathrm{~T} & \mathrm{~T} \\ 0.5273587658083839 & \mathrm{~T} & \mathrm{~T} & \mathrm{~T} \\ 0.5337473535513794 & \mathrm{~T} & \mathrm{~T} & \mathrm{~T} \\ 0.5291658759230324 & \mathrm{~T} & \mathrm{~T} & \mathrm{~T} \\ 0.4169820333737184 & \mathrm{~T} & \mathrm{~T} & \mathrm{~T} \\ 0.0000000000000000 & \mathrm{~F} & \mathrm{~F} & \mathrm{~F} \\ 0.2122994622105073 & \mathrm{~T} & \mathrm{~T} & \mathrm{~T} \\ 0.1047483626335719 & \mathrm{~F} & \mathrm{~F} & \mathrm{~F} \\ 0.3165278254398524 & \mathrm{~T} & \mathrm{~T} & \mathrm{~T} \\ 0.1441744226128918 & \mathrm{~F} & \mathrm{~F} & \mathrm{~F}\end{array}$


0.0728008949706867

0.2500657030223365

$-0.0040836527067209$

0.5000000000000000

0.7487378583845521

0.5000000000000000

0.7490280433217932

0.7500657030223365

0.4241233122671250

0.7500657030223365

0.5039328452888746

0.0000000000000000

0.2483172810067082

0.0000000000000000

0.2487157697722905

0.2500657030223365

$-0.0233014323431504$

0.2500657030223365

$-0.0021511891162714$

0.5000000000000000

0.7500004232582755

0.5000000000000000

0.7487522162608020

0.7500657030223365

0.5179533215313981

0.7500657030223365

0.4993902778698655

0.0731701301616822

0.2491384976448501

0.4285017878778895

0.1857671520580378

$-0.0016921842687242$

0.2500657030223365

0.0000000000000000

0.2473846155267213

0.5047797206835437

0.7500657030223365

0.5000000000000000

0.7504414043547974

0.0005183951857772

0.2500657030223365

0.0000000000000000

0.2469173075284361

0.4953078925443935

0.7500657030223365

0.5000000000000000

0.7493846808492401
0.3826985067684923

0.2500657030223365

$-0.0048280321152455$

0.0000000000000000

0.2471208582420143

0.2500657030223365

0.0311954981472891

0.0000000000000000

0.3373400135415947

0.2500657030223365

$-0.0087171717955330$

0.5000000000000000

0.7529019020372598

0.7500657030223365

0.5605270957006897

0.5000000000000000

0.7526774882301887

0.7500657030223365

0.4909921941293997

0.5000000000000000

0.7493155744658831

0.7500657030223365

0.4888735829399775

0.5000000000000000

0.7485141374390253

0.7500657030223365

0.4887379951715882

0.0632638065060983

0.2978973064176120

0.0428572658906787

0.6055617977982075

$-0.0225645436385349$

0.2500657030223365

0.2500657030223365

0.0008818502423341

$-0.0273738292239092$

0.2500657030223365

0.2500657030223365

0.0016056531863966

0.5321685199906346

0.7500657030223365

0.7500657030223365

0.5020879002072062

0.5180538941929892

0.7500657030223365

0.7500657030223365

0.4971747222217510
0.3844860477957941

0.0394260599793199

0.2526516998935281

0.0000000000000000

0.2109854145747030

0.1047483626335719

0.3243410587783570

0.1441744226128918

0.3735925325233019

0.0394260599793199

0.2526023382720368

0.0000000000000000

0.2100120935072158

0.1047483626335719

0.3090985625776921

0.1441744226128918

0.3599820790978591

0.0394260599793199

0.2464610656839382

0.0000000000000000

0.2125112214544458

0.1047483626335719

0.3163215859139789

0.1441744226128918

0.3573133921886397

0.0394260599793199

0.2467278393388927

0.4113166838186361

0.4825986046164072

0.4169554616401371

0.5568934396975650

0.3413324083541658

0.1244398483281657

0.0197345742847261

0.2321900256299738

0.3387648556787201

0.1244398483281657

0.0197345742847261

0.2312458885580972

0.3308557586832640

0.1244398483281657

0.0197345742847261

0.2284251267488694

0.3296700900194969

0.1244398483281657

0.0197345742847261

0.2292865129338474
F $F \quad F$

T T T

$\begin{array}{lll}F & F & F\end{array}$

$\begin{array}{lll}\mathrm{T} & \mathrm{T} & \mathrm{T}\end{array}$

F $F \quad F$

$\begin{array}{lll}T & T & T\end{array}$

$\begin{array}{lll}F & F & F\end{array}$

$\begin{array}{lll}\text { T } & T & T\end{array}$

F $\quad F \quad F$

T T T

F $F \quad$ F

T $T$ T

F $\quad F \quad F$

T $T$ T

F $F \quad F$

T $T$ T

F $F \quad F$

T T $T$

F $\quad F \quad F$

$\begin{array}{lll}T & T & T\end{array}$

F F F

T $T$ T

F $F \quad F$

T $T$ T

F $F \quad F$

T $T$ T

T T T

T $T$ T

T $T$ T

T $T$ T

T $T$ T

F $F \quad F$

F $\quad F \quad F$

T $T$ T

T T T

$\begin{array}{lll}\text { F } & F & F\end{array}$

$\begin{array}{lll}\text { F } & F & F\end{array}$

T $T$ T

T T T

F $F \quad F$

$\begin{array}{lll}F & F & F\end{array}$

T $T$ T

T T T

F $\quad F \quad F$

F $F \quad F$

T T T

\section{3}

\section{$\begin{array}{lllll}\text { H } & \mathrm{C} & \mathrm{V} & \mathrm{O} & \mathrm{Ti}\end{array}$ \\ $\begin{array}{lllll}5 & 1 & 1 & 36 & 16\end{array}$}

0.4504435606355318

0.1003326978082529

0.3310223664153972

0.1814732718409558

0.0761921862541749

0.6795115616287047

0.6450877925805090

0.6309695446827791

0.2627024749890881

0.2877521481555224

0.4453254368864612

0.4917797796768763

0.5028350998338791

0.5624032501049305

0.5158662784343785

$\begin{array}{lll}\mathrm{T} & \mathrm{T} & \mathrm{T} \\ \mathrm{T} & \mathrm{T} & \mathrm{T} \\ \mathrm{T} & \mathrm{T} & \mathrm{T} \\ \mathrm{T} & \mathrm{T} & \mathrm{T} \\ \mathrm{T} & \mathrm{T} & \mathrm{T}\end{array}$


0.1982245871041008

0.1991862837282144

0.0000000000000000

0.2485603607470613

0.0000000000000000

0.2627661603263470

0.2500657030223365

0.0412149297477185

0.2500657030223365

0.0043278808337471

0.5000000000000000

0.7483939897999317

0.5000000000000000

0.7518100060175729

0.7500657030223365

0.3879773413390202

0.7500657030223365

0.5061650744761939

0.0000000000000000

0.2487927194865720

0.0000000000000000

0.2357944740290044

0.2500657030223365

0.9747036668879754

0.2500657030223365

0.9888007113070261

0.5000000000000000

0.7480965312662849

0.5000000000000000

0.7444235843486937

0.7500657030223365

0.5149619009166808

0.7500657030223365

0.4950252583817632

0.0670861033135949

0.2913168697336401

0.5126186873632022

0.1706246025242174

0.0042949391454518

0.2500657030223365

0.0000000000000000

0.2604895642174698

0.5097325226311062

0.7500657030223365

0.5000000000000000

0.7508174743083422

0.9888242370828801

0.2500657030223365

0.0000000000000000

0.2365438271740970

0.4910632806982917

0.7500657030223365

0.5000000000000000

0.7452359855090046
0.6037958174532486

0.2921500403024724

0.0000000000000000

0.2501377096848660

0.2500657030223365

0.9859145207559215

0.0000000000000000

0.4620808026417233

0.2500657030223365

0.0044958137432496

0.0000000000000000

0.2496118192979998

0.2500657030223365

0.0220090738330343

0.0000000000000000

0.3576174786451494

0.2500657030223365

0.9975149212611903

0.5000000000000000

0.7499990521641667

0.7500657030223365

0.5214996808246166

0.5000000000000000

0.7680936771848194

0.7500657030223365

0.5008285452403233

0.5000000000000000

0.7489709813046587

0.7500657030223365

0.4669572382090534

0.5000000000000000

0.7231566365964199

0.7500657030223365

0.4926100105481049

0.1340785207436570

0.1650818947398543

0.0308959815965298

0.4204875695208189

0.9896822927851942

0.2500657030223365

0.2500657030223365

0.9997034957033880

0.9571450279408518

0.2500657030223365

0.2500657030223365

0.0004056406586399

0.5393353989782860

0.7500657030223365

0.7500657030223365

0.5002537919038851

0.5021601086192027

0.7500657030223365

0.7500657030223365

0.4956614564110342
0.5161454050049613

0.4296927895006175

0.0000000000000000

0.2120168327238278

0.1047483626335719

0.3155882232650559

0.1441744226128918

0.4083372188924411

0.0394260599793199

0.2496964411849011

0.0000000000000000

0.2105092861554326

0.1047483626335719

0.3173092439129008

0.1441744226128918

0.3924997113659831

0.0394260599793199

0.2476357803219697

0.0000000000000000

0.2108955776145949

0.1047483626335719

0.3127130540740353

0.1441744226128918

0.3556357769423483

0.0394260599793199

0.2483637177149305

0.0000000000000000

0.2124940666651306

0.1047483626335719

0.3205418317675769

0.1441744226128918

0.3545580563231448

0.0394260599793199

0.2503052704409215

0.3938704166301749

0.4859186835746745

0.4103581460785986

0.5069793236648878

0.3330085619641528

0.1244398483281657

0.0197345742847261

0.2305401298521837

0.3386636498610671

0.1244398483281657

0.0197345742847261

0.2301908062671155

0.3373034840613499

0.1244398483281657

0.0197345742847261

0.2299967642299117

0.3331553663213896

0.1244398483281657

0.0197345742847261

0.2299380395581540

\begin{tabular}{|c|c|}
\hline $\mathrm{T}$ & $\mathrm{T}$ \\
\hline $\mathrm{T}$ & $\mathrm{T}$ \\
\hline$F$ & $\mathrm{~F}$ \\
\hline $\mathrm{T}$ & $\mathrm{T}$ \\
\hline$F$ & $\mathrm{~F}$ \\
\hline $\mathrm{T}$ & $\mathrm{T}$ \\
\hline$F$ & $F$ \\
\hline $\mathrm{T}$ & $\mathrm{T}$ \\
\hline $\mathrm{F}$ & $F$ \\
\hline $\mathrm{T}$ & $\mathrm{T}$ \\
\hline $\mathrm{F}$ & $\mathrm{F}$ \\
\hline $\mathrm{T}$ & $\mathrm{T}$ \\
\hline $\mathrm{F}$ & $F$ \\
\hline $\mathrm{T}$ & $\mathrm{T}$ \\
\hline $\mathrm{F}$ & $\mathrm{F}$ \\
\hline $\mathrm{T}$ & $\mathrm{T}$ \\
\hline $\mathrm{F}$ & $\mathrm{F}$ \\
\hline $\mathrm{T}$ & $\mathrm{T}$ \\
\hline $\mathrm{F}$ & $\mathrm{F}$ \\
\hline $\mathrm{T}$ & $\mathrm{T}$ \\
\hline $\mathrm{F}$ & $\mathrm{F}$ \\
\hline $\mathrm{T}$ & $\mathrm{T}$ \\
\hline $\mathrm{F}$ & $\mathrm{F}$ \\
\hline $\mathrm{T}$ & $\mathrm{T}$ \\
\hline $\mathrm{F}$ & $\mathrm{F}$ \\
\hline $\mathrm{T}$ & $\mathrm{T}$ \\
\hline $\mathrm{F}$ & $\mathrm{F}$ \\
\hline $\mathrm{T}$ & $\mathrm{T}$ \\
\hline$F$ & $F$ \\
\hline $\mathrm{T}$ & $\mathrm{T}$ \\
\hline $\mathrm{F}$ & $\mathrm{F}$ \\
\hline $\mathrm{T}$ & $\mathrm{T}$ \\
\hline $\mathrm{F}$ & $\mathrm{F}$ \\
\hline $\mathrm{T}$ & $\mathrm{T}$ \\
\hline $\mathrm{T}$ & $\mathrm{T}$ \\
\hline $\mathrm{T}$ & $\mathrm{T}$ \\
\hline $\mathrm{T}$ & $\mathrm{T}$ \\
\hline $\mathrm{T}$ & $\mathrm{T}$ \\
\hline $\mathrm{T}$ & $\mathrm{T}$ \\
\hline$F$ & $\mathrm{~F}$ \\
\hline $\mathrm{F}$ & $\mathrm{F}$ \\
\hline $\mathrm{T}$ & $\mathrm{T}$ \\
\hline $\mathrm{T}$ & $\mathrm{T}$ \\
\hline $\mathrm{F}$ & $\mathrm{F}$ \\
\hline $\mathrm{F}$ & $F$ \\
\hline $\mathrm{T}$ & $\mathrm{T}$ \\
\hline $\mathrm{T}$ & $\mathrm{T}$ \\
\hline$F$ & $F$ \\
\hline $\mathrm{F}$ & $\mathrm{F}$ \\
\hline $\mathrm{T}$ & $\mathrm{T}$ \\
\hline $\mathrm{T}$ & $\mathrm{T}$ \\
\hline$F$ & $F$ \\
\hline $\mathrm{F}$ & $F$ \\
\hline & \\
\hline
\end{tabular}




\section{$\begin{array}{lllll}H & C & \text { V } & \text { O } & \text { Ti }\end{array}$ $\begin{array}{lllll}5 & 1 & 1 & 36 & 16\end{array}$}

0.4293542714743666

0.0927395348932194

0.3273643615517514

0.1976566326485667

0.3155166478907105

0.2047076193723214

0.1963511887877445

0.0000000000000000

0.2521642752725697

0.0000000000000000

0.2665652049386935

0.2500657030223365

0.0349924756780950

0.2500657030223365

0.0087904227201250

0.5000000000000000

0.7511909170064541

0.5000000000000000

0.7565933903622479

0.7500657030223365

0.3833744751231194

0.7500657030223365

0.5091011636930958

0.0000000000000000

0.2514123416443468

0.0000000000000000

0.2371730144147790

0.2500657030223365

0.9813240105974685

0.2500657030223365

0.9899554087498998

0.5000000000000000

0.7502610767320377

0.5000000000000000

0.7442531305002049

0.7500657030223365

0.5141591913582957

0.7500657030223365

0.4965943556639374

0.0664292854830677

0.2753724903352435

0.5200673234221682

0.1993521809715020

0.0122655632555180

0.2500657030223365

0.0000000000000000

0.2624723650604537

0.5149035114632028

0.7500657030223365

0.5000000000000000

0.7562281029120541

0.9885736085408183

0.2500657030223365
0.0550308223831451

0.6547835057135387

0.6478661395678884

0.6225178687036887

0.1379606198074796

0.5934802213586252

0.2986202920040668

0.0000000000000000

0.2513836695410134

0.2500657030223365

0.9757284568623348

0.0000000000000000

0.4506109968607745

0.2500657030223365

0.0105022337695857

0.0000000000000000

0.2508349245214276

0.2500657030223365

0.0089036045971582

0.0000000000000000

0.3664198981490188

0.2500657030223365

0.0023110927362069

0.5000000000000000

0.7495079902039166

0.7500657030223365

0.5057294885574848

0.5000000000000000

0.7594036389992880

0.7500657030223365

0.5051050915397113

0.5000000000000000

0.7493057751112602

0.7500657030223365

0.4621092463968708

0.5000000000000000

0.7162297784094491

0.7500657030223365

0.4971583173887240

0.1298959964573854

0.0976730855532537

0.0396209559478606

0.4077136728375805

0.9865111421193262

0.2500657030223365

0.2500657030223365

0.0005221964987996

0.9499233239566962

0.2500657030223365

0.2500657030223365

0.0009538041971595

0.5344613772487179

0.7500657030223365
0.4387160687326883

0.4877850337247978

0.4922844846032604

0.5565533275097465

0.5153344151772430

0.5101921589344798

0.4340213793048022

0.0000000000000000

0.2117155716475665

0.1047483626335719

0.3150533799475213

0.1441744226128918

0.4085334681205950

0.0394260599793199

0.2496698170464384

0.0000000000000000

0.2105410822384073

0.1047483626335719

0.3164048471962317

0.1441744226128918

0.3988191405017361

0.0394260599793199

0.2469074207159920

0.0000000000000000

0.2109625478912207

0.1047483626335719

0.3135054060542188

0.1441744226128918

0.3558848845297859

0.0394260599793199

0.2485545041347805

0.0000000000000000

0.2123338500232563

0.1047483626335719

0.3207885784732554

0.1441744226128918

0.3568677724749136

0.0394260599793199

0.2510352501085436

0.3930572723747190

0.4776691195171523

0.4059923189325545

0.5031437296173422

0.3343777901972373

0.1244398483281657

0.0197345742847261

0.2303564349502594

0.3366435650552262

0.1244398483281657

0.0197345742847261

0.2304261253046462

0.3361724636424270

0.1244398483281657
T $T$ T

$\begin{array}{lll}T & T & T\end{array}$

$\begin{array}{lll}T & T & T\end{array}$

T $T$ T

T $T$ T

T T T

T T $T$

F $\quad F \quad F$

T $T$ T

F $F \quad F$

T $T$ T

F $\quad F \quad F$

T $T$ T

F $F \quad$ F

T $T$ T

F $\quad F \quad F$

T T T

F $\quad F \quad F$

T $T$ T

F $F \quad F$

T $T$ T

F $F \quad F$

$\begin{array}{lll}T & T & T\end{array}$

F $F \quad F$

T $T$ T

F $F \quad F$

T $T$ T

F $\quad F \quad F$

T $T$ T

$\begin{array}{lll}F & F & F\end{array}$

T T T

F $F \quad F$

T $T$ T

F $\quad F \quad F$

T $T$ T

F $F \quad F$

T T T

F $\quad F \quad F$

T $T$ T

T $T$ T

$\begin{array}{lll}T & T & T\end{array}$

T $T$ T

T $T$ T

T T T

F $F \quad F$

$\begin{array}{lll}F & F & F\end{array}$

T $T$ T

T $T$ T

F $F \quad F$

F $\quad F \quad F$

T $T$ T

$\begin{array}{lll}\mathrm{T} & \mathrm{T} & \mathrm{T}\end{array}$

F F F 
0.0000000000000000

0.2395283895602205

0.4918899953251739

0.7500657030223365

0.5000000000000000

0.7454040999193481
0.7500657030223365

0.5005815809874576

0.4980242919797107

0.7500657030223365

0.7500657030223365

0.4963007322939674
0.0197345742847261

0.2300926335686384

0.3346108631052047

0.1244398483281657

0.0197345742847261

0.2297073062484642

$\begin{array}{lll}\mathrm{F} & \mathrm{F} & \mathrm{F} \\ \mathrm{T} & \mathrm{T} & \mathrm{T} \\ \mathrm{T} & \mathrm{T} & \mathrm{T} \\ \mathrm{F} & \mathrm{F} & \mathrm{F} \\ \mathrm{F} & \mathrm{F} & \mathrm{F} \\ \mathrm{T} & \mathrm{T} & \mathrm{T}\end{array}$

\section{5}

\section{$\begin{array}{llllll}\text { H } & \mathbf{C} & \mathrm{V} & \mathrm{O} & \mathrm{Ti}\end{array}$ $\begin{array}{lllll}5 & 1 & 1 & 36 & 16\end{array}$}

0.4090278557324865

0.0203004835221504

0.1571014944278199

$-0.0259605772110732$

0.2190796550639535

0.0945775534859468

0.1960231831388673

0.0000000000000000

0.2530699682595304

0.0000000000000000

0.2668735233096419

0.2500657030223364

0.0247810920215024

0.2500657030223364

0.0075940566247981

0.4999999999999808

0.7498778049562007

0.4999999999999808

0.7526051992208119

0.7500657030223350

0.3969381231680091

0.7500657030223350

0.5075204360803726

0.0000000000000000

0.2501496590569665

0.0000000000000000

0.2396036908187419

0.2500657030223364

0.9839597298675001

0.2500657030223364

0.9915155826003477

0.4999999999999808

0.7514609113724655

0.4999999999999808

0.7457467771349667

0.7500657030223350

0.5170770749593554

0.7500657030223350

0.4973983868900107

0.0527685446599017

0.2070088859044101

0.5183162040577676

0.2057681850080663

0.0106422086393146

0.2500657030223364

0.0000000000000000
0.0478434036322191

0.5687109634634216

0.6377450542418656

0.4734773396797018

0.0844372364863961

0.5308784303156706

0.3158649495671245

0.0000000000000000

0.2510969926374693

0.2500657030223364

0.9802181725681661

0.0000000000000000

0.4924929807240809

0.2500657030223364

0.0125581581982547

0.0000000000000000

0.2505175370517249

0.2500657030223364

0.0135464568827946

0.0000000000000000

0.3726545742447551

0.2500657030223364

$-0.0007600380129332$

0.4999999999999808

0.7500469002064104

0.7500657030223350

0.5177412533912559

0.4999999999999808

0.7651278835065424

0.7500657030223350

0.5029424932369895

0.4999999999999808

0.7494085891246682

0.7500657030223350

0.4693523706403641

0.4999999999999808

0.7229272739055833

0.7500657030223350

0.4958310414795124

0.2141915563742747

0.0851679048815952

0.0420366272042415

0.4218982117202842

1.0061325337884219

0.2500657030223364

0.2500657030223364
0.4317855839796121

0.4589804645084460

0.5488266036506076

0.5427352021667053

0.5002149259268246

0.5247052174045278

0.4284361572624920

0.0000000000000000

0.2117994635360297

0.1047483626335719

0.3157627650598112

0.1441744226128918

0.4195687128886770

0.0394260599793199

0.2486201208637721

0.0000000000000000

0.2105410608484624

0.1047483626335719

0.3158714457438925

0.1441744226128918

0.3983659354915072

0.0394260599793199

0.2458127227832338

0.0000000000000000

0.2108842389017739

0.1047483626335719

0.3138984503737998

0.1441744226128918

0.3504625818575578

0.0394260599793199

0.2490037725913153

0.0000000000000000

0.2122327055109071

0.1047483626335719

0.3207344433637266

0.1441744226128918

0.3571171146581429

0.0394260599793199

0.2508752029026670

0.3664853511403335

0.4584247161737914

0.4075205949652184

0.4980418179841043

0.3301955060750721

0.1244398483281657

0.0197345742847261

$\begin{array}{lll}\mathrm{T} & \mathrm{T} & \mathrm{T} \\ \mathrm{T} & \mathrm{T} & \mathrm{T} \\ \mathrm{T} & \mathrm{T} & \mathrm{T} \\ \mathrm{T} & \mathrm{T} & \mathrm{T} \\ \mathrm{T} & \mathrm{T} & \mathrm{T} \\ \mathrm{T} & \mathrm{T} & \mathrm{T} \\ \mathrm{T} & \mathrm{T} & \mathrm{T} \\ \mathrm{F} & \mathrm{F} & \mathrm{F} \\ \mathrm{T} & \mathrm{T} & \mathrm{T} \\ \mathrm{F} & \mathrm{F} & \mathrm{F} \\ \mathrm{T} & \mathrm{T} & \mathrm{T} \\ \mathrm{F} & \mathrm{F} & \mathrm{F} \\ \mathrm{T} & \mathrm{T} & \mathrm{T} \\ \mathrm{F} & \mathrm{F} & \mathrm{F} \\ \mathrm{T} & \mathrm{T} & \mathrm{T} \\ \mathrm{F} & \mathrm{F} & \mathrm{F} \\ \mathrm{T} & \mathrm{T} & \mathrm{T} \\ \mathrm{F} & \mathrm{F} & \mathrm{F} \\ \mathrm{T} & \mathrm{T} & \mathrm{T} \\ \mathrm{F} & \mathrm{F} & \mathrm{F} \\ \mathrm{T} & \mathrm{T} & \mathrm{T} \\ \mathrm{F} & \mathrm{F} & \mathrm{F} \\ \mathrm{T} & \mathrm{T} & \mathrm{T} \\ \mathrm{F} & \mathrm{F} & \mathrm{F} \\ \mathrm{T} & \mathrm{T} & \mathrm{T} \\ \mathrm{F} & \mathrm{F} & \mathrm{F} \\ \mathrm{T} & \mathrm{T} & \mathrm{T} \\ \mathrm{F} & \mathrm{F} & \mathrm{F} \\ \mathrm{T} & \mathrm{T} & \mathrm{T} \\ \mathrm{F} & \mathrm{F} & \mathrm{F} \\ \mathrm{T} & \mathrm{T} & \mathrm{T} \\ \mathrm{F} & \mathrm{F} & \mathrm{F} \\ \mathrm{T} & \mathrm{T} & \mathrm{T} \\ \mathrm{F} & \mathrm{F} & \mathrm{F} \\ \mathrm{T} & \mathrm{T} & \mathrm{T} \\ \mathrm{F} & \mathrm{F} & \mathrm{F} \\ \mathrm{T} & \mathrm{T} & \mathrm{T} \\ \mathrm{F} & \mathrm{F} & \mathrm{F} \\ \mathrm{T} & \mathrm{T} & \mathrm{T} \\ \mathrm{T} & \mathrm{T} & \mathrm{T} \\ \mathrm{T} & \mathrm{T} & \mathrm{T} \\ \mathrm{T} & \mathrm{T} & \mathrm{T} \\ \mathrm{T} & \mathrm{T} & \mathrm{T} \\ \mathrm{T} & \mathrm{T} & \mathrm{T} \\ \mathrm{F} & \mathrm{F} & \mathrm{F} \\ \mathrm{F} & \mathrm{F} & \mathrm{F} \\ & & \end{array}$


0.2639171244640479

0.5136628981559577

0.7500657030223350

0.4999999999999808

0.7525026633916261

0.9917491122666491

0.2500657030223364

0.0000000000000000

0.2402212544503201

0.4942920153065576

0.7500657030223350

0.4999999999999808

0.7471047271082248

6

\section{$\begin{array}{lllll}\text { H } & \text { C } & \text { V } & \text { O } & \text { Ti }\end{array}$ \\ $\begin{array}{lllll}5 & 1 & 1 & 36 & 16\end{array}$}

0.4115807831925672

0.2301926646935865

0.2663245829980175

0.4603638136489988

0.0205339815326475

0.3224894965151525

0.1941691705100629

0.0000000000000000

0.2526833072367103

0.0000000000000000

0.2661473532449365

0.2500657030223365

0.0115319580836140

0.2500657030223365

0.0078611251480212

0.5000000000000000

0.7492116761660047

0.5000000000000000

0.7541231620258290

0.7500657030223365

0.3921366314739601

0.7500657030223365

0.5072543234132150

0.0000000000000000

0.2499324963223024

0.0000000000000000

0.2389090233416564

0.2500657030223365

0.9877956505389746

0.2500657030223365

0.9905075935822615

0.5000000000000000

0.7506370755894300

0.5000000000000000

0.7434519756583781

0.7500657030223365

0.5130163014399760

0.7500657030223365

0.4964509739134141
0.9996070069316646

0.9538889204280204

0.2500657030223364

0.2500657030223364

$-0.0000703684000085$

0.5365035515496650

0.7500657030223350

0.7500657030223350

0.5000643247387520

0.5036421681652042

0.7500657030223350

0.7500657030223350

0.4956101699831151
0.2298689137002908

0.3367498792259379

0.1244398483281657

0.0197345742847261

0.2297478134409853

0.3373230014030556

0.1244398483281657

0.0197345742847261

0.2306982696436792

0.3348697751070971

0.1244398483281657

0.0197345742847261

0.2304985483847949

$\begin{array}{lll}\mathrm{T} & \mathrm{T} & \mathrm{T} \\ \mathrm{T} & \mathrm{T} & \mathrm{T} \\ \mathrm{F} & \mathrm{F} & \mathrm{F} \\ \mathrm{F} & \mathrm{F} & \mathrm{F} \\ \mathrm{T} & \mathrm{T} & \mathrm{T} \\ \mathrm{T} & \mathrm{T} & \mathrm{T} \\ \mathrm{F} & \mathrm{F} & \mathrm{F} \\ \mathrm{F} & \mathrm{F} & \mathrm{F} \\ \mathrm{T} & \mathrm{T} & \mathrm{T} \\ \mathrm{T} & \mathrm{T} & \mathrm{T} \\ \mathrm{F} & \mathrm{F} & \mathrm{F} \\ \mathrm{F} & \mathrm{F} & \mathrm{F} \\ \mathrm{T} & \mathrm{T} & \mathrm{T}\end{array}$

0.0475250052667960

0.0689384520092862

0.4767918929487415

0.4801243081895357

0.5955721935144839

0.4504750651916170

0.3101133085310862

0.0000000000000000

0.2510994716092688

0.2500657030223365

0.9816171313146645

0.0000000000000000

0.4916355826810048

0.2500657030223365

0.0126512294160437

0.0000000000000000

0.2503870611113034

0.2500657030223365

0.0068457105770684

0.0000000000000000

0.3715987156122565

0.2500657030223365

0.0002965673828937

0.5000000000000000

0.7502119880341795

0.7500657030223365

0.5105417710391618

0.5000000000000000

0.7604446139879626

0.7500657030223365

0.5032604924732539

0.5000000000000000

0.7494969055178254

0.7500657030223365

0.4671513244428276

0.5000000000000000

0.7200199006576785

0.7500657030223365

0.4975392273436792
0.4293554079076287

0.4973701967882774

0.5853921898814107

0.5330390744022280

0.4454953708143170

0.5428015502770754

0.4286007085807576

0.0000000000000000

0.2118251075107887

0.1047483626335719

0.3163689306922567

0.1441744226128918

0.4212089762062327

0.0394260599793199

0.2489539711526385

0.0000000000000000

0.2107678586273344

0.1047483626335719

0.3160063506785226

0.1441744226128918

0.3998167382985723

0.0394260599793199

0.2464513625500428

0.0000000000000000

0.2112200418349861

0.1047483626335719

0.3139305936886766

0.1441744226128918

0.3505945371859673

0.0394260599793199

0.2488790929316379

0.0000000000000000

0.2123173757787291

0.1047483626335719

0.3206517835071650

0.1441744226128918

0.3576624293889368

0.0394260599793199

0.2507610467322026

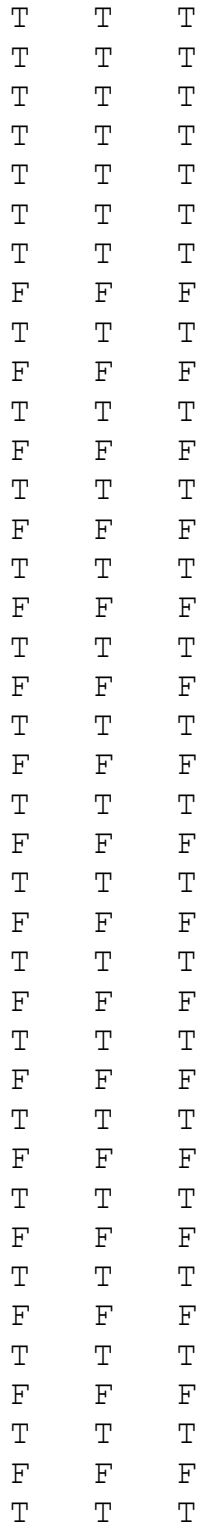


0.0458911364657770

0.2138188848542971

0.5238180402851131

0.2227537815193633

0.0111909538509121

0.2500657030223365

0.0000000000000000

0.2628234620852222

0.5139433683978470

0.7500657030223365

0.5000000000000000

0.7519949115919802

0.9910970865629621

0.2500657030223365

0.0000000000000000

0.2403994995164538

0.4933677041530115

0.7500657030223365

0.5000000000000000

0.7458589639189864
0.2171972661871986

0.0743798351124813

0.0446134836728554

0.3889209887034505

0.0039646214807346

0.2500657030223365

0.2500657030223365

0.0002910686251873

0.9511506346474710

0.2500657030223365

0.2500657030223365

0.0004362558760126

0.5312883291295022

0.7500657030223365

0.7500657030223365

0.5004040783890180

0.5016571097258474

0.7500657030223365

0.7500657030223365

0.4964884492512161
0.3667681094161955

0.4556973603391243

0.4060475647921652

0.5032514207416449

0.3303056136413862

0.1244398483281657

0.0197345742847261

0.2295133507420431

0.3359674344766433

0.1244398483281657

0.0197345742847261

0.2294274184255989

0.3358969875298994

0.1244398483281657

0.0197345742847261

0.2300696131858942

0.3344493410194973

0.1244398483281657

0.0197345742847261

0.2297507431162654

$\begin{array}{lll}\mathrm{T} & \mathrm{T} & \mathrm{T} \\ \mathrm{T} & \mathrm{T} & \mathrm{T} \\ \mathrm{T} & \mathrm{T} & \mathrm{T} \\ \mathrm{T} & \mathrm{T} & \mathrm{T} \\ \mathrm{T} & \mathrm{T} & \mathrm{T} \\ \mathrm{F} & \mathrm{F} & \mathrm{F} \\ \mathrm{F} & \mathrm{F} & \mathrm{F} \\ \mathrm{T} & \mathrm{T} & \mathrm{T} \\ \mathrm{T} & \mathrm{T} & \mathrm{T} \\ \mathrm{F} & \mathrm{F} & \mathrm{F} \\ \mathrm{F} & \mathrm{F} & \mathrm{F} \\ \mathrm{T} & \mathrm{T} & \mathrm{T} \\ \mathrm{T} & \mathrm{T} & \mathrm{T} \\ \mathrm{F} & \mathrm{F} & \mathrm{F} \\ \mathrm{F} & \mathrm{F} & \mathrm{F} \\ \mathrm{T} & \mathrm{T} & \mathrm{T} \\ \mathrm{T} & \mathrm{T} & \mathrm{T} \\ \mathrm{F} & \mathrm{F} & \mathrm{F} \\ \mathrm{F} & \mathrm{F} & \mathrm{F} \\ \mathrm{T} & \mathrm{T} & \mathrm{T}\end{array}$

\section{7}

\section{$\begin{array}{llll}H & \mathrm{~V} & \mathrm{O} & \mathrm{Ti}\end{array}$ \\ $\begin{array}{llll}3 & 1 & 35 & 16\end{array}$}

0.4037901230072900

0.2140089699687552

0.9578219926316884

0.2582303689400527

0.0000000000000000

0.2506263621371287

0.0000000000000000

0.2544923235412538

0.2500657030223365

0.0604571672222477

0.2500657030223365

0.9974961584658349

0.5000000000000000

0.7498911067349887

0.5000000000000000

0.7492005490405599

0.7500657030223365

0.4294546895717496

0.7500657030223365

0.5063008967348146

0.0000000000000000

0.2504459566173305

0.0000000000000000

0.2449713540999031

0.2500657030223365

0.9762790114849892

0.2500657030223365

0.9973568601358164

0.5000000000000000

0.7507070451519031

0.5000000000000000

0.7549601112814470
0.9219894010271943

0.3988066008365162

0.3524974696073644

0.2201049396169380

0.0000000000000000

0.2493116693949878

0.2500657030223365

0.0048921540243336

0.0000000000000000

0.4071312377564151

0.2500657030223365

0.0000382401420171

0.0000000000000000

0.2483439572583737

0.2500657030223365

0.0310510144804887

0.0000000000000000

0.3202576381164404

0.2500657030223365

0.9924975174604368

0.5000000000000000

0.7520088973010374

0.7500657030223365

0.5367161674039038

0.5000000000000000

0.7541164140771458

0.7500657030223365

0.4989954656010395

0.5000000000000000

0.7496105041690890

0.7500657030223365

0.4772327919975044
0.4419837688091348

0.5023540307864025

0.4190146584789392

0.4178594330459969

0.0000000000000000

0.2124824340526153

0.1047483626335719

0.3161457772479131

0.1441744226128918

0.4010531642808540

0.0394260599793199

0.2516777966504773

0.0000000000000000

0.2110238846255960

0.1047483626335719

0.3249177133572489

0.1441744226128918

0.3724024437772624

0.0394260599793199

0.2519888645582380

0.0000000000000000

0.2111836410225180

0.1047483626335719

0.3122833963064962

0.1441744226128918

0.3567905428752459

0.0394260599793199

0.2466350760058273

0.0000000000000000

0.2126701207488276

0.1047483626335719

0.3191058507645722

$\begin{array}{ccc}\text { T } & \text { T } & \text { T } \\ \text { T } & \text { T } & \text { T } \\ \text { T } & \text { T } & \text { T } \\ \text { T } & \text { T } & \text { T } \\ \text { F } & \text { F } & \text { F } \\ \text { T } & \text { T } & \text { T } \\ \text { F } & \text { F } & \text { F } \\ \text { T } & \text { T } & \text { T } \\ \text { F } & \text { F } & \text { F } \\ \text { T } & \text { T } & \text { T } \\ \text { F } & \text { F } & \text { F } \\ \text { T } & \text { T } & \text { T } \\ \text { F } & \text { F } & \text { F } \\ \text { T } & \text { T } & \text { T } \\ \text { F } & \text { F } & \text { F } \\ \text { T } & \text { T } & \text { T } \\ \text { F } & \text { F } & \text { F } \\ \text { T } & \text { T } & \text { T } \\ \text { F } & \text { F } & \text { F } \\ \text { T } & \text { T } & \text { T } \\ \text { F } & \text { F } & \text { F } \\ \text { T } & \text { T } & \text { T } \\ \text { F } & \text { F } & \text { F } \\ \text { T } & \text { T } & \text { T } \\ \text { F } & \text { F } & \text { F } \\ \text { T } & \text { T } & \text { T } \\ \text { F } & \text { F } & \text { F } \\ \text { T } & \text { T } & \text { T } \\ \text { F } & \text { F } & \text { F } \\ \text { T } & \text { T } & \text { T } \\ \text { F } & \text { F } & \text { F } \\ \text { T } & \text { T } & \text { T } \\ & & \end{array}$


0.7500657030223365

0.5178255578023870

0.7500657030223365

0.5015478020485059

0.0700707791999650

0.2770819900212241

0.4332237249247493

0.9976325570789866

0.2500657030223365

0.0000000000000000

0.2519795082828753

0.5062130700601487

0.7500657030223365

0.5000000000000000

0.7518449869217683

$-0.0004738102492022$

0.2500657030223365

0.0000000000000000

0.2476288216140635

0.4996580584815442

0.7500657030223365

0.5000000000000000

0.7517882372790605
0.5000000000000000

0.7423607554226921

0.7500657030223365

0.4931401161080900

0.0795230126616312

0.2924365917799577

0.0266775479137096

0.9813368072119659

0.2500657030223365

0.2500657030223365

0.0001382579829614

0.9695615253243232

0.2500657030223365

0.2500657030223365

0.0009963223419970

0.5350939496543144

0.7500657030223365

0.7500657030223365

0.5009191233523373

0.5100242633550187

0.7500657030223365

0.7500657030223365

0.4969929195169429
0.1441744226128918

0.3550903223091363

0.0394260599793199

0.2476614385983398

0.4064087875400064

0.4910514957271545

0.4198549406747053

0.3375032169153308

0.1244398483281657

0.0197345742847261

0.2312068613691952

0.3372317486636779

0.1244398483281657

0.0197345742847261

0.2293854354967362

0.3307323811700764

0.1244398483281657

0.0197345742847261

0.2282328253793654

0.3293141856257367

0.1244398483281657

0.0197345742847261

0.2280454285526596

\begin{tabular}{|c|c|}
\hline$F$ & $F$ \\
\hline $\mathrm{T}$ & $\mathrm{T}$ \\
\hline $\mathrm{F}$ & $F$ \\
\hline $\mathrm{T}$ & $\mathrm{T}$ \\
\hline $\mathrm{T}$ & $\mathrm{T}$ \\
\hline $\mathrm{T}$ & $\mathrm{T}$ \\
\hline $\mathrm{T}$ & $\mathrm{T}$ \\
\hline $\mathrm{T}$ & $\mathrm{T}$ \\
\hline $\mathrm{F}$ & $F$ \\
\hline $\mathrm{F}$ & $\mathrm{F}$ \\
\hline $\mathrm{T}$ & $\mathrm{T}$ \\
\hline $\mathrm{T}$ & $\mathrm{T}$ \\
\hline $\mathrm{F}$ & $\mathrm{F}$ \\
\hline $\mathrm{F}$ & $\mathrm{F}$ \\
\hline $\mathrm{T}$ & $\mathrm{T}$ \\
\hline $\mathrm{T}$ & $\mathrm{T}$ \\
\hline $\mathrm{F}$ & $\mathrm{F}$ \\
\hline $\mathrm{F}$ & $\mathrm{F}$ \\
\hline $\mathrm{T}$ & $\mathrm{T}$ \\
\hline $\mathrm{T}$ & $\mathrm{T}$ \\
\hline $\mathrm{F}$ & $\mathrm{F}$ \\
\hline $\mathrm{F}$ & $F$ \\
\hline T & $\mathrm{T}$ \\
\hline
\end{tabular}

8

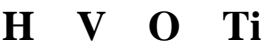 \\ $\begin{array}{llll}3 & 1 & 35 & 16\end{array}$}

0.3452402883731875

0.1329712984516666

0.4549358170531872

0.2559547887743091

0.0000000000000000

0.2502457927283995

0.0000000000000000

0.2493001408830109

0.2500657030223364

0.0654888472445224

0.2500657030223364

0.9995486343327686

0.4999999999999942

0.7485581278786525

0.4999999999999942

0.7500602729053294

0.7500657030223350

0.4379731072536865

0.7500657030223350

0.5014957421873992

0.0000000000000000

0.2482296195212627

0.0000000000000000

0.2467067420044683

0.2500657030223364

0.9758258347653829

0.2500657030223364

0.9978524561434194

0.4999999999999942
0.3872424352410331 0.3966806348387549

0.9512152905326964

0.1747347127323206

0.0000000000000000

0.2482084800865552

0.2500657030223364

0.0359406714573385

0.0000000000000000

0.3793434972559299

0.2500657030223364

0.9897214015748618

0.0000000000000000

0.2459017732750283

0.2500657030223364

0.0286220960593205

0.0000000000000000

0.3119241637607889

0.2500657030223364

0.9906779937996606

0.4999999999999942

0.7530864168707990

0.7500657030223350

0.5485932566854377

0.4999999999999942

0.7449575415072849

0.7500657030223350

0.4878798996334933

0.4999999999999942
0.4806775158880209

0.4351756014331323

0.4575227563246459

0.3996016597249348

0.0000000000000000

0.2117064800674568

0.1047483626335719

0.3200814781191635

0.1441744226128918

0.3898519435821906

0.0394260599793199

0.2524890145001089

0.0000000000000000

0.2111606647532943

0.1047483626335719

0.3237750819225119

0.1441744226128918

0.3640269775101208

0.0394260599793199

0.2522863225822232

0.0000000000000000

0.2104119999934052

0.1047483626335719

0.3094539661857035

0.1441744226128918

0.3567705126592007

0.0394260599793199

0.2461101433369251

0.0000000000000000

$\begin{array}{ccc}\text { T } & \text { T } & \text { T } \\ \mathrm{T} & \mathrm{T} & \mathrm{T} \\ \mathrm{T} & \mathrm{T} & \mathrm{T} \\ \mathrm{T} & \mathrm{T} & \mathrm{T} \\ \mathrm{F} & \mathrm{F} & \mathrm{F} \\ \mathrm{T} & \mathrm{T} & \mathrm{T} \\ \mathrm{F} & \mathrm{F} & \mathrm{F} \\ \mathrm{T} & \mathrm{T} & \mathrm{T} \\ \mathrm{F} & \mathrm{F} & \mathrm{F} \\ \mathrm{T} & \mathrm{T} & \mathrm{T} \\ \mathrm{F} & \mathrm{F} & \mathrm{F} \\ \mathrm{T} & \mathrm{T} & \mathrm{T} \\ \mathrm{F} & \mathrm{F} & \mathrm{F} \\ \mathrm{T} & \mathrm{T} & \mathrm{T} \\ \mathrm{F} & \mathrm{F} & \mathrm{F} \\ \mathrm{T} & \mathrm{T} & \mathrm{T} \\ \mathrm{F} & \mathrm{F} & \mathrm{F} \\ \mathrm{T} & \mathrm{T} & \mathrm{T} \\ \mathrm{F} & \mathrm{F} & \mathrm{F} \\ \mathrm{T} & \mathrm{T} & \mathrm{T} \\ \mathrm{F} & \mathrm{F} & \mathrm{F} \\ \mathrm{T} & \mathrm{T} & \mathrm{T} \\ \mathrm{F} & \mathrm{F} & \mathrm{F} \\ \mathrm{T} & \mathrm{T} & \mathrm{T} \\ \mathrm{F} & \mathrm{F} & \mathrm{F} \\ \mathrm{T} & \mathrm{T} & \mathrm{T} \\ \mathrm{F} & \mathrm{F} & \mathrm{F} \\ \mathrm{T} & \mathrm{T} & \mathrm{T} \\ \mathrm{F} & \mathrm{F} & \mathrm{F} \\ & & \end{array}$


0.7510311098616340

0.4999999999999942

0.7498194537121120

0.7500657030223350

0.5046493899246504

0.7500657030223350

0.5001009759691777

0.0714960513531567

0.2398174847250021

0.4597522593347984

0.9879936587354483

0.2500657030223364

0.0000000000000000

0.2528995218053957

0.4994483064796170

0.7500657030223350

0.4999999999999942

0.7477023090870089

0.0013945025776252

0.2500657030223364

0.0000000000000000

0.2461502967211911

0.4973851025003975

0.7500657030223350

0.4999999999999942

0.7511445805440427
0.7495866009071668

0.7500657030223350

0.4861185329625801

0.4999999999999942

0.7460139853303525

0.7500657030223350

0.4942699968774434

0.0423775077727102

0.3235762611777653

0.0319897724315723

0.9712176463281482

0.2500657030223364

0.2500657030223364

0.0009773389367533

0.9771092959406682

0.2500657030223364

0.2500657030223364

0.9992378586382310

0.5227048799326510

0.7500657030223350

0.7500657030223350

0.5010397680491118

0.5142630956740192

0.7500657030223350

0.7500657030223350

0.4958460836798722
0.2123886736968260

0.1047483626335719

0.3158350598448578

0.1441744226128918

0.3562037655277946

0.0394260599793199

0.2451826213826015

0.4110661777333104

0.4695385102427609

0.4251912511151560

0.3376631291800541

0.1244398483281657

0.0197345742847261

0.2307586443793470

0.3413286002411056

0.1244398483281657

0.0197345742847261

0.2307319590178977

0.3312085118980943

0.1244398483281657

0.0197345742847261

0.2280335613771839

0.3273865839663207

0.1244398483281657

0.0197345742847261

0.2286339581955695

$\begin{array}{lll}\text { T } & \text { T } & \text { T } \\ \text { F } & \text { F } & \text { F } \\ \text { T } & \text { T } & \text { T } \\ \text { F } & \text { F } & \text { F } \\ \text { T } & \text { T } & \text { T } \\ \text { F } & \text { F } & \text { F } \\ \text { T } & \text { T } & \text { T } \\ \text { T } & \text { T } & \text { T } \\ \text { T } & \text { T } & \text { T } \\ \text { T } & \text { T } & \text { T } \\ \text { T } & \text { T } & \text { T } \\ \text { F } & \text { F } & \text { F } \\ \text { F } & \text { F } & \text { F } \\ \text { T } & \text { T } & \text { T } \\ \text { T } & \text { T } & \text { T } \\ \text { F } & \text { F } & \text { F } \\ \text { F } & \text { F } & \text { F } \\ \text { T } & \text { T } & \text { T } \\ \text { T } & \text { T } & \text { T } \\ \text { F } & \text { F } & \text { F } \\ \text { F } & \text { F } & \text { F } \\ \text { T } & \text { T } & \text { T } \\ \text { T } & \text { T } & \text { T } \\ \text { F } & \text { F } & \text { F } \\ \text { F } & \text { F } & \text { F } \\ \text { T } & \text { T } & \text { T }\end{array}$

9

\section{$\begin{array}{llll}H & \mathrm{~V} & \mathrm{O} & \mathrm{Ti}\end{array}$ \\ $\begin{array}{llll}3 & 1 & 35 & 16\end{array}$}

0.4419888535892367

0.1677381992162335

0.3653400591260640

0.2463262497679276

0.0000000000000000

0.2487902001079346

0.0000000000000000

0.2500133622773476

0.2500657030223365

0.0677324184480274

0.2500657030223365

0.0010336282218677

0.5000000000000000

0.7511981228511667

0.5000000000000000

0.7515218196662141

0.7500657030223365

0.4390649402114344

0.7500657030223365

0.5014953018510537

0.0000000000000000

0.2517573349095408

0.0000000000000000

0.2491502506714435

0.2500657030223365

0.9815097574822401
0.9436995965660645

0.4171781730189850

0.3705071179378009

0.1796263379674284

0.0000000000000000

0.2477653467348348

0.2500657030223365

0.0413376715362052

0.0000000000000000

0.3531079967474206

0.2500657030223365

0.9848360002115240

0.0000000000000000

0.2449397103121303

0.2500657030223365

0.0266091310816523

0.0000000000000000

0.3273203298683201

0.2500657030223365

0.9929873390504048

0.5000000000000000

0.7537702058406582

0.7500657030223365

0.5562822783419941

0.5000000000000000

0.7448311768758754
0.4506291188462077

0.4676483466003505

0.4810491033685549

0.3959672436426899

0.0000000000000000

0.2116689088241685

0.1047483626335719

0.3214739737882146

0.1441744226128918

0.3776700542997280

0.0394260599793199

0.2540874042590255

0.0000000000000000

0.2114860312436488

0.1047483626335719

0.3251349877787812

0.1441744226128918

0.3692360518339635

0.0394260599793199

0.2533975876176938

0.0000000000000000

0.2100556886721888

0.1047483626335719

0.3086686719221026

0.1441744226128918

0.3588586607153380

$\begin{array}{lll}\text { T } & \text { T } & \text { T } \\ \text { T } & \text { T } & \text { T } \\ \text { T } & \text { T } & \text { T } \\ \text { T } & \text { T } & \text { T } \\ \text { F } & \text { F } & \text { F } \\ \text { T } & \text { T } & \text { T } \\ \text { F } & \text { F } & \text { F } \\ \text { T } & \text { T } & \text { T } \\ \text { F } & \text { F } & \text { F } \\ \text { T } & \text { T } & \text { T } \\ \text { F } & \text { F } & \text { F } \\ \text { T } & \text { T } & \text { T } \\ \text { F } & \text { F } & \text { F } \\ \text { T } & \text { T } & \text { T } \\ \text { F } & \text { F } & \text { F } \\ \text { T } & \text { T } & \text { T } \\ \text { F } & \text { F } & \text { F } \\ \text { T } & \text { T } & \text { T } \\ \text { F } & \text { F } & \text { F } \\ \text { T } & \text { T } & \text { T } \\ \text { F } & \text { F } & \text { F } \\ \text { T } & \text { T } & \text { T } \\ \text { F } & \text { F } & \text { F } \\ \text { T } & \text { T } & \text { T } \\ \text { F } & \text { F } & \text { F } \\ \text { T } & \text { T } & \text { T }\end{array}$


0.2500657030223365

$-0.0012369689121486$

0.5000000000000000

0.7490622480020237

0.5000000000000000

0.7484431026421013

0.7500657030223365

0.5036221539970782

0.7500657030223365

0.5000439217452731

0.0818272299337744

0.2483858192980904

0.4644499777558426

0.9899854081540839

0.2500657030223365

0.0000000000000000

0.2516284382828899

0.5028529764708544

0.7500657030223365

0.5000000000000000

0.7487861426113785

0.0025346903554093

0.2500657030223365

0.0000000000000000

0.2484602021018942

0.4963448347313699

0.7500657030223365

0.5000000000000000

0.7504121787403690
0.7500657030223365

0.4894025113185587

0.5000000000000000

0.7500929599374518

0.7500657030223365

0.4937471303408081

0.5000000000000000

0.7490481095372618

0.7500657030223365

0.4905746040834961

0.0342407279772467

0.3201464295839260

0.0425917343840026

0.9689641012984366

0.2500657030223365

0.2500657030223365

0.0014334291470035

0.9780344037687998

0.2500657030223365

0.2500657030223365

0.9981802850904005

0.5195516269824291

0.7500657030223365

0.7500657030223365

0.5018409819861193

0.5196512222447310

0.7500657030223365

0.7500657030223365

0.4952395907158895
0.0394260599793199

0.2449320680922109

0.0000000000000000

0.2123640704937189

0.1047483626335719

0.3152557489710220

0.1441744226128918

0.3588513093381612

0.0394260599793199

0.2451293767949868

0.4158016224907372

0.4761841126913334

0.4251173902534676

0.3400831569908213

0.1244398483281657

0.0197345742847261

0.2306121268971146

0.3420131859986800

0.1244398483281657

0.0197345742847261

0.2312388002858506

0.3291435209834634

0.1244398483281657

0.0197345742847261

0.2277531259558165

0.3279107277866697

0.1244398483281657

0.0197345742847261

0.2286157968315810

$\begin{array}{lll}\mathrm{F} & \mathrm{F} & \mathrm{F} \\ \mathrm{T} & \mathrm{T} & \mathrm{T} \\ \mathrm{F} & \mathrm{F} & \mathrm{F} \\ \mathrm{T} & \mathrm{T} & \mathrm{T} \\ \mathrm{F} & \mathrm{F} & \mathrm{F} \\ \mathrm{T} & \mathrm{T} & \mathrm{T} \\ \mathrm{F} & \mathrm{F} & \mathrm{F} \\ \mathrm{T} & \mathrm{T} & \mathrm{T} \\ \mathrm{F} & \mathrm{F} & \mathrm{F} \\ \mathrm{T} & \mathrm{T} & \mathrm{T} \\ \mathrm{T} & \mathrm{T} & \mathrm{T} \\ \mathrm{T} & \mathrm{T} & \mathrm{T} \\ \mathrm{T} & \mathrm{T} & \mathrm{T} \\ \mathrm{T} & \mathrm{T} & \mathrm{T} \\ \mathrm{F} & \mathrm{F} & \mathrm{F} \\ \mathrm{F} & \mathrm{F} & \mathrm{F} \\ \mathrm{T} & \mathrm{T} & \mathrm{T} \\ \mathrm{T} & \mathrm{T} & \mathrm{T} \\ \mathrm{F} & \mathrm{F} & \mathrm{F} \\ \mathrm{F} & \mathrm{F} & \mathrm{F} \\ \mathrm{T} & \mathrm{T} & \mathrm{T} \\ \mathrm{T} & \mathrm{T} & \mathrm{T} \\ \mathrm{F} & \mathrm{F} & \mathrm{F} \\ \mathrm{F} & \mathrm{F} & \mathrm{F} \\ \mathrm{T} & \mathrm{T} & \mathrm{T} \\ \mathrm{T} & \mathrm{T} & \mathrm{T} \\ \mathrm{F} & \mathrm{F} & \mathrm{F} \\ \mathrm{F} & \mathrm{F} & \mathrm{F} \\ \mathrm{T} & \mathrm{T} & \mathrm{T} \\ & & \end{array}$

10

\section{$\begin{array}{llll}\text { H } & \mathbf{V} & \mathbf{O} & \mathbf{T i}\end{array}$ \\ $\begin{array}{llll}1 & 1 & 34 & 16\end{array}$}

0.5528774295612960

0.2456941987420807

0.0000000000000000

0.2441485083987941

0.0000000000000000

0.2564612667054941

0.2500657030223365

0.0587185860168662

0.2500657030223365

0.0027204841391305

0.5000000000000000

0.7517287256031240

0.5000000000000000

0.7515114798626619

0.7500657030223365

0.4156255698826627

0.7500657030223365

0.5021474169886239

0.0000000000000000

0.2525908661344324

0.0000000000000000

0.2451339475321903

0.2500657030223365
0.1504882571591668

0.1761531401901620

0.0000000000000000

0.2475532091295129

0.2500657030223365

0.0356912175355014

0.0000000000000000

0.3296533744199240

0.2500657030223365

0.9844236606947045

0.0000000000000000

0.2451728464647630

0.2500657030223365

0.0280367649646395

0.0000000000000000

0.3633103085883989

0.2500657030223365

0.9950429978255695

0.5000000000000000

0.7537111057129031

0.7500657030223365

0.5642209814127528

0.5000000000000000
0.4355877931889698

0.3899540547111316

0.0000000000000000

0.2117072716380077

0.1047483626335719

0.3218355031850977

0.1441744226128918

0.3657941635080793

0.0394260599793199

0.2549033655450708

0.0000000000000000

0.2114079051814423

0.1047483626335719

0.3244935121572556

0.1441744226128918

0.3812402670168653

0.0394260599793199

0.2525239082376043

0.0000000000000000

0.2096651781558081

0.1047483626335719

0.3081382516696871

0.1441744226128918

\begin{tabular}{|c|c|}
\hline $\mathrm{T}$ & $\mathrm{T}$ \\
\hline $\mathrm{T}$ & $\mathrm{T}$ \\
\hline $\mathrm{F}$ & $\mathrm{F}$ \\
\hline $\mathrm{T}$ & $\mathrm{T}$ \\
\hline $\mathrm{F}$ & $\mathrm{F}$ \\
\hline $\mathrm{T}$ & $\mathrm{T}$ \\
\hline $\mathrm{F}$ & $\mathrm{F}$ \\
\hline $\mathrm{T}$ & $\mathrm{T}$ \\
\hline $\mathrm{F}$ & $\mathrm{F}$ \\
\hline $\mathrm{T}$ & $\mathrm{T}$ \\
\hline $\mathrm{F}$ & $\mathrm{F}$ \\
\hline $\mathrm{T}$ & $\mathrm{T}$ \\
\hline $\mathrm{F}$ & $\mathrm{F}$ \\
\hline $\mathrm{T}$ & $\mathrm{T}$ \\
\hline $\mathrm{F}$ & $\mathrm{F}$ \\
\hline $\mathrm{T}$ & $\mathrm{T}$ \\
\hline $\mathrm{F}$ & $\mathrm{F}$ \\
\hline $\mathrm{T}$ & $\mathrm{T}$ \\
\hline $\mathrm{F}$ & $\mathrm{F}$ \\
\hline $\mathrm{T}$ & $\mathrm{T}$ \\
\hline $\mathrm{F}$ & $\mathrm{F}$ \\
\hline $\mathrm{T}$ & $\mathrm{T}$ \\
\hline
\end{tabular}


0.9821954460015563

0.2500657030223365

$-0.0054971418030491$

0.5000000000000000

0.7454377474969057

0.5000000000000000

0.7433585267420554

0.7500657030223365

0.5098161605355843

0.7500657030223365

0.4952081327261345

0.0912078587486395

0.4759483473745552

0.9901360696179481

0.2500657030223365

0.0000000000000000

0.2511370928884901

0.5071077340848542

0.7500657030223365

0.5000000000000000

0.7495505860598120

$-0.0036951699119600$

0.2500657030223365

0.0000000000000000

0.2463109393247724

0.4879861479728777

0.7500657030223365

0.5000000000000000

0.7431494665659457
0.7509861020083486

0.7500657030223365

0.4938218329314762

0.5000000000000000

0.7500723625138582

0.7500657030223365

0.4944642167352630

0.5000000000000000

0.7461971194776553

0.7500657030223365

0.4847016425673453

0.0430319943589298

0.0621566632214168

0.9748863081499708

0.2500657030223365

0.2500657030223365

0.0006045850824443

0.9695243454405477

0.2500657030223365

0.2500657030223365

$-0.0019579573995206$

0.5214477422541733

0.7500657030223365

0.7500657030223365

0.5017651857777792

0.5236847950648844

0.7500657030223365

0.7500657030223365

0.4945690641591006
0.3592067976278173

0.0394260599793199

0.2447467538986925

0.0000000000000000

0.2124963184858460

0.1047483626335719

0.3156311183173017

0.1441744226128918

0.3607959830871098

0.0394260599793199

0.2463058129284145

0.4203383009407187

0.4187853530095448

0.3411096378709733

0.1244398483281657

0.0197345742847261

0.2295378005069385

0.3405713877329595

0.1244398483281657

0.0197345742847261

0.2310547058286224

0.3277421946682024

0.1244398483281657

0.0197345742847261

0.2280069741878593

0.3308858141807620

0.1244398483281657

0.0197345742847261

0.2291672813875916

$\begin{array}{lll}\text { T } & \text { T } & \text { T } \\ \text { F } & \text { F } & \text { F } \\ \text { T } & \text { T } & \text { T } \\ \text { F } & \text { F } & \text { F } \\ \text { T } & \text { T } & \text { T } \\ \text { F } & \text { F } & \text { F } \\ \text { T } & \text { T } & \text { T } \\ \text { F } & \text { F } & \text { F } \\ \text { T } & \text { T } & \text { T } \\ \text { F } & \text { F } & \text { F } \\ \text { T } & \text { T } & \text { T } \\ \text { T } & \text { T } & \text { T } \\ \text { T } & \text { T } & \text { T } \\ \text { T } & \text { T } & \text { T } \\ \text { F } & \text { F } & \text { F } \\ \text { F } & \text { F } & \text { F } \\ \text { T } & \text { T } & \text { T } \\ \text { T } & \text { T } & \text { T } \\ \text { F } & \text { F } & \text { F } \\ \text { F } & \text { F } & \text { F } \\ \text { T } & \text { T } & \text { T } \\ \text { T } & \text { T } & \text { T } \\ \text { F } & \text { F } & \text { F } \\ \text { F } & \text { F } & \text { F } \\ \text { T } & \text { T } & \text { T } \\ \text { T } & \text { T } & \text { T } \\ \text { F } & \text { F } & \text { F } \\ \text { F } & \text { F } & \text { F } \\ \text { T } & \text { T } & \text { T } \\ & & \end{array}$

\section{For Figure 3: 2-15}

\section{2}

\section{$\begin{array}{llllll}\text { H } & \mathbf{C} & \mathbf{V} & \mathbf{O} & \mathrm{Ti}\end{array}$ $\begin{array}{lllll}5 & 1 & 1 & 36 & 16\end{array}$}

$0.4021650417925065-0.0555029365234857$

0.2053136526824790

0.1950218055726342

0.3949485445177621

0.2167545684172112

0.2494191639992666

0.2375688565403317

0.0000000000000000

0.2490834818351992

0.0000000000000000

0.2510269548929346

0.2500657030223365

0.0728008949706867

0.2500657030223365

$-0.0040836527067209$

0.5000000000000000

0.7487378583845521

0.5000000000000000

0.7490280433217932

0.7500657030223365

0.4241233122671250
0.8723790692988502

0.7747409744182425

0.7627847514507866

0.5026976871610860

0.7593796805949922

0.2350468109027780

0.0000000000000000

0.2480040087209304

0.2500657030223365

0.0061705618490033

0.0000000000000000

0.3826985067684923

0.2500657030223365

$-0.0048280321152455$

0.0000000000000000

0.2471208582420143

0.2500657030223365

0.0311954981472891

0.0000000000000000

0.3373400135415947
0.4426074853221137

0.5547977657687174

0.4851370962237689

0.5273587658083839

0.5337473535513794

0.5291658759230324

0.4169820333737184

0.0000000000000000

0.2122994622105073

0.1047483626335719

0.3165278254398524

0.1441744226128918

0.3844860477957941

0.0394260599793199

0.2526516998935281

0.0000000000000000

0.2109854145747030

0.1047483626335719

0.3243410587783570

0.1441744226128918

0.3735925325233019

$\begin{array}{lll}\mathrm{T} & \mathrm{T} & \mathrm{T} \\ \mathrm{T} & \mathrm{T} & \mathrm{T} \\ \mathrm{T} & \mathrm{T} & \mathrm{T} \\ \mathrm{T} & \mathrm{T} & \mathrm{T} \\ \mathrm{T} & \mathrm{T} & \mathrm{T} \\ \mathrm{T} & \mathrm{T} & \mathrm{T} \\ \mathrm{T} & \mathrm{T} & \mathrm{T} \\ \mathrm{F} & \mathrm{F} & \mathrm{F} \\ \mathrm{T} & \mathrm{T} & \mathrm{T} \\ \mathrm{F} & \mathrm{F} & \mathrm{F} \\ \mathrm{T} & \mathrm{T} & \mathrm{T} \\ \mathrm{F} & \mathrm{F} & \mathrm{F} \\ \mathrm{T} & \mathrm{T} & \mathrm{T} \\ \mathrm{F} & \mathrm{F} & \mathrm{F} \\ \mathrm{T} & \mathrm{T} & \mathrm{T} \\ \mathrm{F} & \mathrm{F} & \mathrm{F} \\ \mathrm{T} & \mathrm{T} & \mathrm{T} \\ \mathrm{F} & \mathrm{F} & \mathrm{F} \\ \mathrm{T} & \mathrm{T} & \mathrm{T} \\ \mathrm{F} & \mathrm{F} & \mathrm{F} \\ \mathrm{T} & \mathrm{T} & \mathrm{T}\end{array}$


$0.7500657030223365 \quad 0.2500657030223365$

$0.5039328452888746-0.0087171717955330$

$0.0000000000000000 \quad 0.5000000000000000$

$0.2483172810067082 \quad 0.7529019020372598$

$0.0000000000000000 \quad 0.7500657030223365$

$0.2487157697722905 \quad 0.5605270957006897$

$0.2500657030223365 \quad 0.5000000000000000$

$-0.0233014323431504$

0.2500657030223365

$-0.0021511891162714$

0.5000000000000000

0.7500004232582755

0.5000000000000000

0.7487522162608020

0.7500657030223365

0.5179533215313981

0.7500657030223365

0.4993902778698655

0.0731701301616822

0.2491384976448501

0.4285017878778895

0.1857671520580378

$-0.0016921842687242$

0.2500657030223365

0.0000000000000000

0.2473846155267213

0.5047797206835437

0.7500657030223365

0.5000000000000000

0.7504414043547974

0.0005183951857772

0.2500657030223365

0.0000000000000000

0.2469173075284361

0.4953078925443935

0.7500657030223365

0.5000000000000000

0.7493846808492401
0.7526774882301887

0.7500657030223365

0.4909921941293997

0.5000000000000000

0.7493155744658831

0.7500657030223365

0.4888735829399775

0.5000000000000000

0.7485141374390253

0.7500657030223365

0.4887379951715882

0.0632638065060983

0.2978973064176120

0.0428572658906787

0.6055617977982075

$-0.0225645436385349$

0.2500657030223365

0.2500657030223365

0.0008818502423341

$-0.0273738292239092$

0.2500657030223365

0.2500657030223365

0.0016056531863966

0.5321685199906346

0.7500657030223365

0.7500657030223365

0.5020879002072062

0.5180538941929892

0.7500657030223365

0.7500657030223365

0.4971747222217510
0.0394260599793199

0.2526023382720368

0.0000000000000000

0.2100120935072158

0.1047483626335719

0.3090985625776921

0.1441744226128918

0.3599820790978591

0.0394260599793199

0.2464610656839382

0.0000000000000000

0.2125112214544458

0.1047483626335719

0.3163215859139789

0.1441744226128918

0.3573133921886397

0.0394260599793199

0.2467278393388927

0.4113166838186361

0.4825986046164072

0.4169554616401371

0.5568934396975650

0.3413324083541658

0.1244398483281657

0.0197345742847261

0.2321900256299738

0.3387648556787201

0.1244398483281657

0.0197345742847261

0.2312458885580972

0.3308557586832640

0.1244398483281657

0.0197345742847261

0.2284251267488694

0.3296700900194969

0.1244398483281657

0.0197345742847261

0.2292865129338474

$\begin{array}{lll}\mathrm{F} & \mathrm{F} & \mathrm{F} \\ \mathrm{T} & \mathrm{T} & \mathrm{T} \\ \mathrm{F} & \mathrm{F} & \mathrm{F} \\ \mathrm{T} & \mathrm{T} & \mathrm{T} \\ \mathrm{F} & \mathrm{F} & \mathrm{F} \\ \mathrm{T} & \mathrm{T} & \mathrm{T} \\ \mathrm{F} & \mathrm{F} & \mathrm{F} \\ \mathrm{T} & \mathrm{T} & \mathrm{T} \\ \mathrm{F} & \mathrm{F} & \mathrm{F} \\ \mathrm{T} & \mathrm{T} & \mathrm{T} \\ \mathrm{F} & \mathrm{F} & \mathrm{F} \\ \mathrm{T} & \mathrm{T} & \mathrm{T} \\ \mathrm{F} & \mathrm{F} & \mathrm{F} \\ \mathrm{T} & \mathrm{T} & \mathrm{T} \\ \mathrm{F} & \mathrm{F} & \mathrm{F} \\ \mathrm{T} & \mathrm{T} & \mathrm{T} \\ \mathrm{F} & \mathrm{F} & \mathrm{F} \\ \mathrm{T} & \mathrm{T} & \mathrm{T} \\ \mathrm{T} & \mathrm{T} & \mathrm{T} \\ \mathrm{T} & \mathrm{T} & \mathrm{T} \\ \mathrm{T} & \mathrm{T} & \mathrm{T} \\ \mathrm{T} & \mathrm{T} & \mathrm{T} \\ \mathrm{T} & \mathrm{T} & \mathrm{T} \\ \mathrm{F} & \mathrm{F} & \mathrm{F} \\ \mathrm{F} & \mathrm{F} & \mathrm{F} \\ \mathrm{T} & \mathrm{T} & \mathrm{T} \\ \mathrm{T} & \mathrm{T} & \mathrm{T} \\ \mathrm{F} & \mathrm{F} & \mathrm{F} \\ \mathrm{F} & \mathrm{F} & \mathrm{F} \\ \mathrm{T} & \mathrm{T} & \mathrm{T} \\ \mathrm{T} & \mathrm{T} & \mathrm{T} \\ \mathrm{F} & \mathrm{F} & \mathrm{F} \\ \mathrm{F} & \mathrm{F} & \mathrm{F} \\ \mathrm{T} & \mathrm{T} & \mathrm{T} \\ \mathrm{T} & \mathrm{T} & \mathrm{T} \\ \mathrm{F} & \mathrm{F} & \mathrm{F} \\ \mathrm{F} & \mathrm{F} & \mathrm{F} \\ & \mathrm{T} & \mathrm{T} \\ & \end{array}$

3

\section{$\begin{array}{lllll}\text { H } & \text { C } & \text { V } & \text { O } & \text { Ti }\end{array}$ $\begin{array}{lllll}5 & 1 & 1 & 36 & 16\end{array}$}

0.4504435606355318

0.1003326978082529

0.3310223664153972

0.1814732718409558

0.2627024749890881

0.1982245871041008

0.1991862837282144

0.0000000000000000

0.2485603607470613

0.0000000000000000

0.2627661603263470

0.2500657030223365

0.0412149297477185

0.2500657030223365

0.0761921862541749

0.6795115616287047

0.6450877925805090

0.6309695446827791

0.2877521481555224

0.6037958174532486

0.2921500403024724

0.0000000000000000

0.2501377096848660

0.2500657030223365

0.9859145207559215

0.0000000000000000

0.4620808026417233

0.2500657030223365
0.4453254368864612 0.4917797796768763 0.5028350998338791 0.5624032501049305 0.5158662784343785 0.5161454050049613 0.4296927895006175 0.0000000000000000 0.2120168327238278 0.1047483626335719 0.3155882232650559 0.1441744226128918 0.4083372188924411 0.0394260599793199

$\begin{array}{lll}\mathrm{T} & \mathrm{T} & \mathrm{T} \\ \mathrm{T} & \mathrm{T} & \mathrm{T} \\ \mathrm{T} & \mathrm{T} & \mathrm{T} \\ \mathrm{T} & \mathrm{T} & \mathrm{T} \\ \mathrm{T} & \mathrm{T} & \mathrm{T} \\ \mathrm{T} & \mathrm{T} & \mathrm{T} \\ \mathrm{T} & \mathrm{T} & \mathrm{T} \\ \mathrm{F} & \mathrm{F} & \mathrm{F} \\ \mathrm{T} & \mathrm{T} & \mathrm{T} \\ \mathrm{F} & \mathrm{F} & \mathrm{F} \\ \mathrm{T} & \mathrm{T} & \mathrm{T} \\ \mathrm{F} & \mathrm{F} & \mathrm{F} \\ \mathrm{T} & \mathrm{T} & \mathrm{T} \\ \mathrm{F} & \mathrm{F} & \mathrm{F}\end{array}$


0.0043278808337471

0.5000000000000000

0.7483939897999317

0.5000000000000000

0.7518100060175729

0.7500657030223365

0.3879773413390202

0.7500657030223365

0.5061650744761939

0.0000000000000000

0.2487927194865720

0.0000000000000000

0.2357944740290044

0.2500657030223365

0.9747036668879754

0.2500657030223365

0.9888007113070261

0.5000000000000000

0.7480965312662849

0.5000000000000000

0.7444235843486937

0.7500657030223365

0.5149619009166808

0.7500657030223365

0.4950252583817632

0.0670861033135949

0.2913168697336401

0.5126186873632022

0.1706246025242174

0.0042949391454518

0.2500657030223365

0.0000000000000000

0.2604895642174698

0.5097325226311062

0.7500657030223365

0.5000000000000000

0.7508174743083422

0.9888242370828801

0.2500657030223365

0.0000000000000000

0.2365438271740970

0.4910632806982917

0.7500657030223365

0.5000000000000000

0.7452359855090046
0.0044958137432496

0.0000000000000000

0.2496118192979998

0.2500657030223365

0.0220090738330343

0.0000000000000000

0.3576174786451494

0.2500657030223365

0.9975149212611903

0.5000000000000000

0.7499990521641667

0.7500657030223365

0.5214996808246166

0.5000000000000000

0.7680936771848194

0.7500657030223365

0.5008285452403233

0.5000000000000000

0.7489709813046587

0.7500657030223365

0.4669572382090534

0.5000000000000000

0.7231566365964199

0.7500657030223365

0.4926100105481049

0.1340785207436570

0.1650818947398543

0.0308959815965298

0.4204875695208189

0.9896822927851942

0.2500657030223365

0.2500657030223365

0.9997034957033880

0.9571450279408518

0.2500657030223365

0.2500657030223365

0.0004056406586399

0.5393353989782860

0.7500657030223365

0.7500657030223365

0.5002537919038851

0.5021601086192027

0.7500657030223365

0.7500657030223365

0.4956614564110342
0.2496964411849011

0.0000000000000000

0.2105092861554326

0.1047483626335719

0.3173092439129008

0.1441744226128918

0.3924997113659831

0.0394260599793199

0.2476357803219697

0.0000000000000000

0.2108955776145949

0.1047483626335719

0.3127130540740353

0.1441744226128918

0.3556357769423483

0.0394260599793199

0.2483637177149305

0.0000000000000000

0.2124940666651306

0.1047483626335719

0.3205418317675769

0.1441744226128918

0.3545580563231448

0.0394260599793199

0.2503052704409215

0.3938704166301749

0.4859186835746745

0.4103581460785986

0.5069793236648878

0.3330085619641528

0.1244398483281657

0.0197345742847261

0.2305401298521837

0.3386636498610671

0.1244398483281657

0.0197345742847261

0.2301908062671155

0.3373034840613499

0.1244398483281657

0.0197345742847261

0.2299967642299117

0.3331553663213896

0.1244398483281657

0.0197345742847261

0.2299380395581540
T $T$ T

$\begin{array}{lll}F & F & F\end{array}$

T T T

F $\quad F \quad F$

T T $T$

F $F \quad F$

T $T$ T

F $F \quad F$

T T T

F $F \quad F$

T $T$ T

F $F \quad F$

T $T$ T

F $\quad F \quad F$

T $T$ T

F $F$ F

T $T$ T

F $F \quad F$

T $T$ T

F $\quad F \quad F$

$\begin{array}{lll}T & T & T\end{array}$

F $F \quad F$

T $T$ T

F $\quad F \quad F$

T $T$ T

T $T$ T

$\begin{array}{lll}T & T & T\end{array}$

T T T

T T $T$

T T $T$

F $F \quad F$

F $F \quad F$

T T T

T $T$ T

F $F \quad F$

F $F \quad F$

T $T$ T

T $T$ T

F $F \quad F$

F $F \quad F$

T $T$ T

T $T$ T

F $F \quad F$

$\begin{array}{lll}F & F & F\end{array}$

T T T

4

\section{$\begin{array}{lllll}H & C & \text { V } & \text { O } & \text { Ti }\end{array}$}

\section{$\begin{array}{lllll}5 & 1 & 1 & 36 & 16\end{array}$}

0.4293542714743666

0.0927395348932194

0.3273643615517514

0.1976566326485667

0.3155166478907105

0.2047076193723214

0.1963511887877445

0.0550308223831451

0.6547835057135387

0.6478661395678884

0.6225178687036887

0.1379606198074796

0.5934802213586252

0.2986202920040668
0.4387160687326883

0.4877850337247978

0.4922844846032604

0.5565533275097465

0.5153344151772430

0.5101921589344798

0.4340213793048022

$\begin{array}{lll}\mathrm{T} & \mathrm{T} & \mathrm{T} \\ \mathrm{T} & \mathrm{T} & \mathrm{T} \\ \mathrm{T} & \mathrm{T} & \mathrm{T} \\ \mathrm{T} & \mathrm{T} & \mathrm{T} \\ \mathrm{T} & \mathrm{T} & \mathrm{T} \\ \mathrm{T} & \mathrm{T} & \mathrm{T} \\ \mathrm{T} & \mathrm{T} & \mathrm{T}\end{array}$


0.0000000000000000

0.2521642752725697

0.0000000000000000

0.2665652049386935

0.2500657030223365

0.0349924756780950

0.2500657030223365

0.0087904227201250

0.5000000000000000

0.7511909170064541

0.5000000000000000

0.7565933903622479

0.7500657030223365

0.3833744751231194

0.7500657030223365

0.5091011636930958

0.0000000000000000

0.2514123416443468

0.0000000000000000

0.2371730144147790

0.2500657030223365

0.9813240105974685

0.2500657030223365

0.9899554087498998

0.5000000000000000

0.7502610767320377

0.5000000000000000

0.7442531305002049

0.7500657030223365

0.5141591913582957

0.7500657030223365

0.4965943556639374

0.0664292854830677

0.2753724903352435

0.5200673234221682

0.1993521809715020

0.0122655632555180

0.2500657030223365

0.0000000000000000

0.2624723650604537

0.5149035114632028

0.7500657030223365

0.5000000000000000

0.7562281029120541

0.9885736085408183

0.2500657030223365

0.0000000000000000

0.2395283895602205

0.4918899953251739

0.7500657030223365

0.5000000000000000

0.7454040999193481
0.0000000000000000

0.2513836695410134

0.2500657030223365

0.9757284568623348

0.0000000000000000

0.4506109968607745

0.2500657030223365

0.0105022337695857

0.0000000000000000

0.2508349245214276

0.2500657030223365

0.0089036045971582

0.0000000000000000

0.3664198981490188

0.2500657030223365

0.0023110927362069

0.5000000000000000

0.7495079902039166

0.7500657030223365

0.5057294885574848

0.5000000000000000

0.7594036389992880

0.7500657030223365

0.5051050915397113

0.5000000000000000

0.7493057751112602

0.7500657030223365

0.4621092463968708

0.5000000000000000

0.7162297784094491

0.7500657030223365

0.4971583173887240

0.1298959964573854

0.0976730855532537

0.0396209559478606

0.4077136728375805

0.9865111421193262

0.2500657030223365

0.2500657030223365

0.0005221964987996

0.9499233239566962

0.2500657030223365

0.2500657030223365

0.0009538041971595

0.5344613772487179

0.7500657030223365

0.7500657030223365

0.5005815809874576

0.4980242919797107

0.7500657030223365

0.7500657030223365

0.4963007322939674
0.0000000000000000

0.2117155716475665

0.1047483626335719

0.3150533799475213

0.1441744226128918

0.4085334681205950

0.0394260599793199

0.2496698170464384

0.0000000000000000

0.2105410822384073

0.1047483626335719

0.3164048471962317

0.1441744226128918

0.3988191405017361

0.0394260599793199

0.2469074207159920

0.0000000000000000

0.2109625478912207

0.1047483626335719

0.3135054060542188

0.1441744226128918

0.3558848845297859

0.0394260599793199

0.2485545041347805

0.0000000000000000

0.2123338500232563

0.1047483626335719

0.3207885784732554

0.1441744226128918

0.3568677724749136

0.0394260599793199

0.2510352501085436

0.3930572723747190

0.4776691195171523

0.4059923189325545

0.5031437296173422

0.3343777901972373

0.1244398483281657

0.0197345742847261

0.2303564349502594

0.3366435650552262

0.1244398483281657

0.0197345742847261

0.2304261253046462

0.3361724636424270

0.1244398483281657

0.0197345742847261

0.2300926335686384

0.3346108631052047

0.1244398483281657

0.0197345742847261

0.2297073062484642
F $\quad F \quad F$

$\begin{array}{lll}T & T & T\end{array}$

F $F \quad F$

T T T

F $\quad F \quad F$

$\begin{array}{lll}T & T & T\end{array}$

F $F \quad F$

T $T$ T

$\begin{array}{lll}\text { F } & \text { F } & \text { F }\end{array}$

$\begin{array}{lll}T & T & T\end{array}$

F $F \quad F$

T $T$ T

F $\quad F \quad F$

T T $T$

F $\quad F \quad F$

T $T$ T

F $\quad F \quad F$

T $T$ T

$\begin{array}{lll}\text { F } & \text { F } & \text { F }\end{array}$

$\begin{array}{lll}\text { T } & T & T\end{array}$

F $F \quad F$

T $T$ T

F $\quad F \quad F$

T $T$ T

F $\quad F \quad F$

$\begin{array}{lll}\mathrm{T} & \mathrm{T} & \mathrm{T}\end{array}$

$\begin{array}{lll}\text { F } & \text { F } & \text { F }\end{array}$

T $T$ T

F $F \quad F$

T $T$ T

F $F \quad F$

T $T$ T

T $T$ T

T T T

T $T$ T

T $T$ T

T $T$ T

F $F \quad F$

F F F

T T $T$

T T $T$

F $F \quad F$

F $F \quad F$

T $T$ T

T $T$ T

F $F \quad F$

F $F \quad F$

T $T$ T

T T T

F F F

$\begin{array}{lll}F & F & F\end{array}$

T T T

\section{5}

H C C Ti O 
0.4511930979660466

0.0250911072525619

0.2470621883218085

0.0833743498321436

0.2115409837664982

0.3031502664229049

0.2978406765058478

0.2718220290981014

0.1290035101976661

0.0138855975055348

0.2500657030223365

0.0000000000000000

0.2600087614177483

0.5155481538105049

0.7500657030223365

0.5000000000000000

0.7594826210273777

0.9867133720192026

0.2500657030223365

0.0000000000000000

0.2415165495326487

0.4878013299066278

0.7500657030223365

0.5000000000000000

0.7438322516225919

0.0000000000000000

0.2544700102611419

0.0000000000000000

0.2657671053627601

0.2500657030223365

0.0277221383654531

0.2500657030223365

0.0102854609330715

0.5000000000000000

0.7495713541015483

0.5000000000000000

0.7603774410681728

0.7500657030223365

0.3771430922009216

0.7500657030223365

0.5093188728679117

0.0000000000000000

0.2506824909166591

0.0000000000000000

0.2370433850794884

0.2500657030223365

0.9907170156136651

0.2500657030223365

0.9891453201236206

0.5000000000000000

0.7498232868861482

0.5000000000000000

0.7405258816721014

0.7500657030223365

0.5046561983329962

0.7500657030223365

0.4953962213878244
0.0782069747627812

0.6208049640426357

0.6517108178478920

0.5683054681422863

0.9874311377902267

0.1295408144244372

0.1950714542128495

0.0932982940445574

0.5685746219129908

0.9817109247639315

0.2500657030223365

0.2500657030223365

0.0019941071695075

0.9477911989645882

0.2500657030223365

0.2500657030223365

0.0009492010907306

0.5300858864442020

0.7500657030223365

0.7500657030223365

0.5010919479750097

0.4997588863983721

0.7500657030223365

0.7500657030223365

0.4971682150082976

0.0000000000000000

0.2525314481551061

0.2500657030223365

0.9726222440663105

0.0000000000000000

0.4368239778979941

0.2500657030223365

0.0153861086148394

0.0000000000000000

0.2519429470591927

0.2500657030223365

0.9865136549913387

0.0000000000000000

0.3850408122945711

0.2500657030223365

0.0056732861333700

0.5000000000000000

0.7493850438677260

0.7500657030223365

0.4924824753234008

0.5000000000000000

0.7527481354524437

0.7500657030223365

0.5066949200491535

0.5000000000000000

0.7489591349384725

0.7500657030223365

0.4660346735368524

0.5000000000000000

0.7192504576805674

0.7500657030223365

0.5026836222333715
0.4286501454146465

0.4958816109345214

0.5209057858504124

0.5691513726481759

0.4625788453740382

0.5713270197090806

0.6503285280362666

0.6166425869010259

0.5242045622648575

0.3348993518651654

0.1244398483281657

0.0197345742847261

0.2299434307993062

0.3329005585667306

0.1244398483281657

0.0197345742847261

0.2299246300559536

0.3366825571317545

0.1244398483281657

0.0197345742847261

0.2306687980833408

0.3378785599056866

0.1244398483281657

0.0197345742847261

0.2303767134373535

0.0000000000000000

0.2115291327882166

0.1047483626335719

0.3147139170294627

0.1441744226128918

0.4085493356972023

0.0394260599793199

0.2486657333242209

0.0000000000000000

0.2110161827724296

0.1047483626335719

0.3141601914643444

0.1441744226128918

0.4066602930663871

0.0394260599793199

0.2471319135905171

0.0000000000000000

0.2112889332187832

0.1047483626335719

0.3146125543134226

0.1441744226128918

0.3584056315757134

0.0394260599793199

0.2496573902790364

0.0000000000000000

0.2118588276767485

0.1047483626335719

0.3219718577087790

0.1441744226128918

0.3602009424822643

0.0394260599793199

0.2530208246876932
$\mathrm{T} \quad \mathrm{T} \quad \mathrm{T}$

$\begin{array}{lll}T & T & T\end{array}$

$\begin{array}{lll}T & T & T\end{array}$

T $\mathrm{T} \quad \mathrm{T}$

T $\quad \mathrm{T} \quad \mathrm{T}$

$\begin{array}{lll}\mathrm{T} & \mathrm{T} & \mathrm{T}\end{array}$

$\begin{array}{lll}T & T & T\end{array}$

$\begin{array}{lll}\mathrm{T} & \mathrm{T} & \mathrm{T}\end{array}$

T $\mathrm{T}$ T

$\begin{array}{lll}T & T & T\end{array}$

F $F \quad F$

F $F \quad F$

T $T$ T

T T $T$

F $F \quad F$

F $F \quad F$

T $T$ T

T T $T$

F $\quad F \quad F$

F $\quad F \quad F$

T T T

T $T$ T

F $F \quad F$

F $F \quad F$

T $T$ T

F $\quad F \quad F$

T T T

F $F \quad F$

T $T$ T

F $\quad F \quad F$

T $T$ T

F $F \quad F$

T $T$ T

F $F \quad F$

T $T$ T

F $\quad F \quad F$

T T T

F $F \quad F$

T $T$ T

F $F \quad F$

T T T

F $\quad F \quad F$

T $T$ T

F $F \quad F$

T $T$ T

F $F \quad F$

T T T

F $F \quad F$

T $T$ T

F F F

T T T

F $F \quad F$

T T T

F $F \quad F$

T $T$ T

F F F

T T T 
0.0535668664237756

0.2840466290644949

0.5385536762856007

0.1697655042158950

0.2111707085997801

0.1922948381500793
0.1227179743260371

0.0906602011432250

0.0771399212599382

0.3930933786924166

0.9501851181145523

0.2996268665059312
0.3935489048826665

0.4700452878899877

0.3957333792468656

0.5080143709059262

0.6292491693046857

0.4382632582878756

$\begin{array}{lll}\mathrm{T} & \mathrm{T} & \mathrm{T} \\ \mathrm{T} & \mathrm{T} & \mathrm{T} \\ \mathrm{T} & \mathrm{T} & \mathrm{T} \\ \mathrm{T} & \mathrm{T} & \mathrm{T} \\ \mathrm{T} & \mathrm{T} & \mathrm{T} \\ \mathrm{T} & \mathrm{T} & \mathrm{T}\end{array}$

6

\section{$\begin{array}{lllll}\text { H } & \mathrm{C} & \mathrm{Ti} & \mathrm{O} & \mathrm{V}\end{array}$}

\section{$\begin{array}{lllll}7 & 2 & 16 & 37 & 1\end{array}$}

0.4258238858850967

0.1321538178416715

0.0663265711569244

0.6861449248607910

0.6951437944013859

0.6516771996673538

0.3272391220752480

0.4412771597977522

0.1279105579843138

0.3300591972451682

0.6297225985000207

0.9990739558773079

0.2500657030223364

0.2500657030223364

$-0.0002787076345909$

0.9551345677250622

0.2500657030223364

0.2500657030223364

0.0007475787613680

0.5417151380284106

0.7500657030223350

0.7500657030223350

0.5004117556829164

0.5049717870910547

0.7500657030223350

0.7500657030223350

0.4963540672884673

0.0000000000000000

0.2513650509759715

0.2500657030223364

0.9766570048220532

0.0000000000000000

0.4779362900111749

0.2500657030223364

0.0110896402084866

0.0000000000000000

0.2510007808424797

0.2500657030223364

1.0150113553112947

0.0000000000000000

0.3706349244817273

0.2500657030223364

$-0.0004246450256491$

0.4999999999999817

0.7496147458140259

0.7500657030223350

0.5185498596678564

0.4999999999999817
0.4367716351481342

0.4715597138997601

0.5193365040070245

0.5469206103046914

0.5739407481421132

0.6012100696493978

0.5192568728223471

0.5768213684456652

0.5104570917099791

0.3308526623118495

0.1244398483281657

0.0197345742847261

0.2298056854947392

0.3366107589820013

0.1244398483281657

0.0197345742847261

0.2297357436674039

0.3391681896525100

0.1244398483281657

0.0197345742847261

0.2308218616743015

0.3347523109731472

0.1244398483281657

0.0197345742847261

0.2304736244731097

0.0000000000000000

0.2119937289067799

0.1047483626335719

0.3147760503423803

0.1441744226128918

0.4144459213015519

0.0394260599793199

0.2483620136677392

0.0000000000000000

0.2106113375173729

0.1047483626335719

0.3157969807945766

0.1441744226128918

0.3976267636404111

0.0394260599793199

0.2461855947883803

0.0000000000000000

0.2108281731796467

0.1047483626335719

0.3140645180174690

0.1441744226128918

\begin{tabular}{lll} 
T & T & T \\
T & T & T \\
T & T & T \\
T & T & T \\
T & T & T \\
T & T & T \\
T & T & T \\
T & T & T \\
T & T & T \\
T & T & T \\
F & F & F \\
F & F & F \\
T & T & T \\
T & T & T \\
F & F & F \\
F & F & F \\
T & T & T \\
T & T & T \\
F & F & F \\
F & F & F \\
T & T & T \\
T & T & T \\
F & F & F \\
F & F & F \\
T & T & T \\
F & F & F \\
T & T & T \\
F & F & F \\
T & T & T \\
F & F & F \\
T & T & T \\
F & F & F \\
T & T & T \\
F & F & F \\
T & T & T \\
F & F & F \\
T & T & T \\
F & F & F \\
T & T & T \\
F & F & F \\
T & T & T \\
F & F & F \\
T & T & T \\
F & F & F \\
T & T & T \\
F & F & F \\
\hline &
\end{tabular}


0.9813601210260746

0.2500657030223364

0.9892927842149473

0.4999999999999817

0.7497855885069928

0.4999999999999817

0.7445935960858896

0.7500657030223350

0.5150717352095356

0.7500657030223350

0.4958725506889544

0.0650309828266822

0.2344584316515455

0.5172939885335107

0.2216329489744283

0.3003893578005236

0.1944133150514072

7

\section{$\begin{array}{lllll}\text { H } & \text { C } & \text { V } & \text { O } & \text { Ti }\end{array}$ $\begin{array}{lllll}7 & 2 & 1 & 37 & 16\end{array}$}

0.4072540496275542

0.1124379915006042

0.2631306411938065

0.3317124595724339

0.2789023570110959

0.1083657136406157

0.3134538596271420

0.2480290154676000

0.2473596391369980

0.2363543277120983

0.0000000000000000

0.2486936638958877

0.0000000000000000

0.2512386064966802

0.2500657030223365

0.0710386133966093

0.2500657030223365

0.9962864336629239

0.5000000000000000

0.7491303934910685

0.5000000000000000

0.7494251663654291

0.7500657030223365

0.4225056609072779

0.7500657030223365

0.5040312615389198

0.0000000000000000

0.2486962193054395

0.0000000000000000

0.2483049459170699

0.2500657030223365

0.9767517589053524

0.2500657030223365

0.9977723140454466

0.5000000000000000
0.7709768932310936

0.7500657030223350

0.5022467324259846

0.4999999999999817

0.7492961895439370

0.7500657030223350

0.4707380131099266

0.4999999999999817

0.7241370013878785

0.7500657030223350

0.4969458664052268

0.1830048882594784

0.1239024598786779

0.0450720078955354

0.4463659783531516

0.1906016740140492

0.3136159231861472
0.3547740558576831

0.0394260599793199

0.2493506159156804

0.0000000000000000

0.2123051495123630

0.1047483626335719

0.3215915933931194

0.1441744226128918

0.3573143391528819

0.0394260599793199

0.2515786010376141

0.3767380441127385

0.4730744097911198

0.4069292716105197

0.5026863898054340

0.5724009845767787

0.4311845345951038

$\begin{array}{lll}\text { T } & \text { T } & \text { T } \\ F & F & F \\ T & T & T \\ F & F & F \\ T & T & T \\ F & F & F \\ T & T & T \\ F & F & F \\ T & T & T \\ F & F & F \\ T & T & T \\ T & T & T \\ T & T & T \\ T & T & T \\ T & T & T \\ T & T & T \\ T & T & T\end{array}$

0.9494463927116361 0.6178596383985910

0.5725249519173392

0.8906551525140273

0.0615631122175192

0.9203615402714107

0.4863780762981528

0.6369953771686920

0.9271609298266832

0.2361101104602688

0.0000000000000000

0.2480014280052110

0.2500657030223365

0.0084865903180752

0.0000000000000000

0.3810885599376082

0.2500657030223365

0.9944186997236772

0.0000000000000000

0.2470733147054924

0.2500657030223365

0.0322687818877406

0.0000000000000000

0.3396388715975699

0.2500657030223365

0.9912120380809022

0.5000000000000000

0.7531160510443666

0.7500657030223365

0.5629943175371196

0.5000000000000000

0.7540202516797568

0.7500657030223365

0.4912430414639144

0.5000000000000000
0.4435765019482227

0.5860238623292873

0.6447023568453600

0.5286657254618967

0.5788279677255791

0.5518798356338921

0.5363536156311273

0.6024822849732505

0.5654938784672887

0.4166872234836852

0.0000000000000000

0.2122707203590207

0.1047483626335719

0.3165738545952608

0.1441744226128918

0.3832353082705204

0.0394260599793199

0.2526651824749753

0.0000000000000000

0.2110249642060648

0.1047483626335719

0.3242555660477364

0.1441744226128918

0.3734057562111707

0.0394260599793199

0.2525599901939688

0.0000000000000000

0.2099135808716932

0.1047483626335719

0.3089008784954769

0.1441744226128918

0.3599557249158141

0.0394260599793199

0.2463235914817296

0.0000000000000000

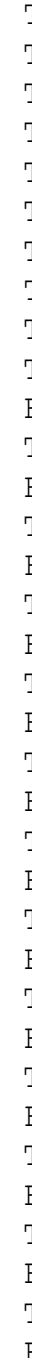


0.7495402028554269

0.5000000000000000

0.7482575878211541

0.7500657030223365

0.5178582482198629

0.7500657030223365

0.4989358502141477

0.0737401857268485

0.2451210124235534

0.4296915914313232

0.3719356532479078

0.2808687293781596

0.9983848282167299

0.2500657030223365

0.0000000000000000

0.2482404317881556

0.5048464385091255

0.7500657030223365

0.5000000000000000

0.7511884488233027

0.0000642054251092

0.2500657030223365

0.0000000000000000

0.2469318442130784

0.4944599247681063

0.7500657030223365

0.5000000000000000

0.7489528300144958
0.7493665077309756

0.7500657030223365

0.4914306683725946

0.5000000000000000

0.7503306806823835

0.7500657030223365

0.4883551382870146

0.0631897085390925

0.3015912803635725

0.0462263956496241

0.5690242661459941

0.8157218165777841

0.9784561539757093

0.2500657030223365

0.2500657030223365

0.0009013301423860

0.9741907940088668

0.2500657030223365

0.2500657030223365

0.0015870010718147

0.5335809415034328

0.7500657030223365

0.7500657030223365

0.5019967656580890

0.5200148330757944

0.7500657030223365

0.7500657030223365

0.4972104083096551
0.2124320561302004

0.1047483626335719

0.3161200737362147

0.1441744226128918

0.3574138629502549

0.0394260599793199

0.2466542102772746

0.4117130179574774

0.4821353552117983

0.4168826376998860

0.5623751361383972

0.6135437971084349

0.3413529721431997

0.1244398483281657

0.0197345742847261

0.2322046476055812

0.3387431224283884

0.1244398483281657

0.0197345742847261

0.2312232686876483

0.3304216860061630

0.1244398483281657

0.0197345742847261

0.2283339187970452

0.3296223600694200

0.1244398483281657

0.0197345742847261

0.2292337091112489

$\begin{array}{lll}\text { T } & \text { T } & \text { T } \\ \text { F } & \text { F } & \text { F } \\ \text { T } & \text { T } & \text { T } \\ \text { F } & \text { F } & \text { F } \\ \text { T } & \text { T } & \text { T } \\ \text { F } & \text { F } & \text { F } \\ \text { T } & \text { T } & \text { T } \\ \text { T } & \text { T } & \text { T } \\ \text { T } & \text { T } & \text { T } \\ \text { T } & \text { T } & \text { T } \\ \text { T } & \text { T } & \text { T } \\ \text { T } & \text { T } & \text { T } \\ \text { T } & \text { T } & \text { T } \\ \text { F } & \text { F } & \text { F } \\ \text { F } & \text { F } & \text { F } \\ \text { T } & \text { T } & \text { T } \\ \text { T } & \text { T } & \text { T } \\ \text { F } & \text { F } & \text { F } \\ \text { F } & \text { F } & \text { F } \\ \text { T } & \text { T } & \text { T } \\ \text { T } & \text { T } & \text { T } \\ \text { F } & \text { F } & \text { F } \\ \text { F } & \text { F } & \text { F } \\ \text { T } & \text { T } & \text { T } \\ \text { T } & \text { T } & \text { T } \\ \text { F } & \text { F } & \text { F } \\ \text { F } & \text { F } & \text { F } \\ \text { T } & \text { T } & \text { T } \\ & & \end{array}$

8

\section{$\begin{array}{llllll}\text { H } & \text { C } & \text { V } & \text { O } & \text { Ti }\end{array}$ $\begin{array}{lllll}7 & 2 & 1 & 37 & 16\end{array}$}

0.4448150018940255

0.2258109990871621

0.9368857084765324

0.9310586519946097

0.2761060928527378

0.1737397977383773

0.0637751749244401

0.0121335135775032

0.1502084065148259

0.1964624248923570

0.0000000000000000

0.2497115835160720

0.0000000000000000

0.2646372661568470

0.2500657030223365

0.0407003175012666

0.2500657030223365

0.0061751746376436

0.5000000000000000

0.7502775105715590

0.5000000000000000

0.7537456783877607

0.7500657030223365

0.3884640155822651

0.0700771859940646

0.2462281147340082

0.5450261365108247

0.3737077658773746

0.7153444815553156

0.8286653153963266

0.8135246082035682

0.4746927006413740

0.7447061157982472

0.2923398979830610

0.0000000000000000

0.2503718704820641

0.2500657030223365

0.9793999579223061

0.0000000000000000

0.4662847535685735

0.2500657030223365

0.0042482036900914

0.0000000000000000

0.2499442826649860

0.2500657030223365

0.0172322288253686

0.0000000000000000

0.3616813128195185
0.4431605276588422

0.5170233876783257

0.5047352297260309

0.5593737190843568

0.5396006367746747

0.5984104762600848

0.5300495554953681

0.5382313359543076

0.5608407323510132

0.4296517399415027

0.0000000000000000

0.2120553332788757

0.1047483626335719

0.3150439474348135

0.1441744226128918

0.4111924542617045

0.0394260599793199

0.2487656976474464

0.0000000000000000

0.2105640621258104

0.1047483626335719

0.3165533360221534

0.1441744226128918

0.3951387935145010

\section{更} $\mathrm{T}$
$\mathrm{F}$
$\mathrm{T}$
$\mathrm{F}$
$\mathrm{T}$
$\mathrm{F}$
$\mathrm{T}$
$\mathrm{T}$
$\mathrm{T}$
$\mathrm{T}$
$\mathrm{T}$
$\mathrm{T}$
$\mathrm{T}$
$\mathrm{F}$
$\mathrm{F}$
$\mathrm{T}$
$\mathrm{T}$
$\mathrm{F}$
$\mathrm{F}$
$\mathrm{T}$
$\mathrm{T}$ 
0.7500657030223365

0.5075588395859759

0.0000000000000000

0.2511734578161768

0.0000000000000000

0.2363148511449771

0.2500657030223365

0.9765031958010085

0.2500657030223365

0.9895079983533931

0.5000000000000000

0.7483337924297483

0.5000000000000000

0.7456860927779168

0.7500657030223365

0.5160787568554229

0.7500657030223365

0.4963004007765383

0.0677679886472804

0.2803056635013609

0.5168618745560875

0.1589979227816817

0.0681164075388178

0.0063499522582017

0.2500657030223365

0.0000000000000000

0.2628788693186404

0.5115894478484619

0.7500657030223365

0.5000000000000000

0.7519639601564678

0.9897913435087705

0.2500657030223365

0.0000000000000000

0.2379540511472530

0.4923101308420341

0.7500657030223365

0.5000000000000000

0.7463376143518191
0.2500657030223365

0.9996112878297926

0.5000000000000000

0.7493483302406315

0.7500657030223365

0.5155786527596811

0.5000000000000000

0.7656014392983689

0.7500657030223365

0.5022600299082339

0.5000000000000000

0.7487805256619938

0.7500657030223365

0.4643958386971648

0.5000000000000000

0.7212085075185728

0.7500657030223365

0.4928656972687832

0.1439509040065096

0.1449992936231303

0.0350354843680142

0.3930882997368049

0.5878413050706687

0.9870749583481617

0.2500657030223365

0.2500657030223365

0.9995789957195231

0.9542150612887432

0.2500657030223365

0.2500657030223365

0.0001703636457900

0.5363949936610745

0.7500657030223365

0.7500657030223365

0.5001139991545965

0.5002178481427361

0.7500657030223365

0.7500657030223365

0.4955238987830740
0.0394260599793199

0.2470833009197353

0.0000000000000000

0.2109612198473357

0.1047483626335719

0.3134329076838068

0.1441744226128918

0.3551730841832552

0.0394260599793199

0.2489309521693525

0.0000000000000000

0.2124548887607267

0.1047483626335719

0.3214637535012667

0.1441744226128918

0.3555099707073239

0.0394260599793199

0.2511900662879874

0.3886636156604245

0.4821737177329372

0.4087457595014871

0.5107397765817033

0.5820173663404155

0.3313494936123478

0.1244398483281657

0.0197345742847261

0.2300738616096644

0.3379529431465013

0.1244398483281657

0.0197345742847261

0.2297390270322491

0.3384567537348319

0.1244398483281657

0.0197345742847261

0.2304317712527579

0.3344126171730023

0.1244398483281657

0.0197345742847261

0.2302372255029244
F F F

T $T$ T

F $F \quad F$

$\begin{array}{lll}T & T & T\end{array}$

$\begin{array}{lll}F & F & F\end{array}$

$\begin{array}{lll}T & T & T\end{array}$

F F F

T $\mathrm{T}$ T

F F F

$\begin{array}{lll}T & T & T\end{array}$

F $F \quad F$

T $T$ T

$\begin{array}{lll}\text { F } & \text { F } & \text { F }\end{array}$

T $T$ T

F $\quad F \quad F$

T T T

F $\quad F \quad F$

$\begin{array}{lll}T & T & T\end{array}$

T T $T$

$\begin{array}{lll}T & T & T\end{array}$

T $T$ T

T $T$ T

T $T$ T

T $T$ T

F $F \quad F$

$\begin{array}{lll}F & F & F\end{array}$

T $T$ T

T $T$ T

F $F \quad F$

$\begin{array}{lll}F & F & F\end{array}$

$\begin{array}{lll}\text { T } & T & T\end{array}$

T T T

$\begin{array}{lll}\text { F } & F & F\end{array}$

F $F \quad F$

T $T$ T

T T $T$

F $\quad F \quad F$

F F F

$\begin{array}{lll}\mathrm{T} & \mathrm{T} & \mathrm{T}\end{array}$
9

\section{$\begin{array}{lllll}\text { H } & \mathbf{C} & \mathrm{V} & \mathrm{O} & \mathrm{Ti}\end{array}$ $\begin{array}{lllll}7 & 2 & 1 & 37 & 16\end{array}$}

0.4334908525958421

0.3300008209787030

$-0.0602042316463123$

$-0.0625256521675356$

0.2788258393547176

0.1687005057594376

0.0648882476421471

0.0165846211122870

0.1507547510182849

0.1872501643344063

0.0000000000000000

0.2504759275613017

0.0000000000000000
0.0662897789258249

0.1286027891499131

0.5372589519360907

0.3598932389495196

0.7100661594646671

0.8160073040008236

0.8030231247039342

0.4650308638090376

0.7346138283693814

0.2962799451280108

0.0000000000000000

0.2519675217441401

0.2500657030223365
0.4364869775117292

0.5128949511585421

0.5016235421850357

0.5544564509742188

0.5394812805522321

0.5984347456563651

0.5289260965613340

0.5346823662713296

0.5599160557449313

0.4348786835032390

0.0000000000000000

0.2117412040700761

0.1047483626335719

$\begin{array}{lll}\mathrm{T} & \mathrm{T} & \mathrm{T} \\ \mathrm{T} & \mathrm{T} & \mathrm{T} \\ \mathrm{T} & \mathrm{T} & \mathrm{T} \\ \mathrm{T} & \mathrm{T} & \mathrm{T} \\ \mathrm{T} & \mathrm{T} & \mathrm{T} \\ \mathrm{T} & \mathrm{T} & \mathrm{T} \\ \mathrm{T} & \mathrm{T} & \mathrm{T} \\ \mathrm{T} & \mathrm{T} & \mathrm{T} \\ \mathrm{T} & \mathrm{T} & \mathrm{T} \\ \mathrm{T} & \mathrm{T} & \mathrm{T} \\ \mathrm{F} & \mathrm{F} & \mathrm{F} \\ \mathrm{T} & \mathrm{T} & \mathrm{T} \\ \mathrm{F} & \mathrm{F} & \mathrm{F}\end{array}$


0.2645646088415152

0.2500657030223365

0.0211382124587753

0.2500657030223365

0.0083518152433333

0.5000000000000000

0.7490842149827821

0.5000000000000000

0.7582282905394482

0.7500657030223365

0.3752920547778764

0.7500657030223365

0.5080179555675897

0.0000000000000000

0.2498624147002004

0.0000000000000000

0.2338955388339448

0.2500657030223365

0.9821756289444952

0.2500657030223365

0.9870612116018856

0.5000000000000000

0.7469382463866208

0.5000000000000000

0.7398289530531669

0.7500657030223365

0.5095728854336212

0.7500657030223365

0.4930913985992944

0.0676711198032780

0.2933325467021621

0.5244034575919447

0.1631696720343508

0.0712268143926099

0.0127245227950224

0.2500657030223365

0.0000000000000000

0.2609884684878350

0.5142237781005918

0.7500657030223365

0.5000000000000000

0.7550330013627753

0.9843839912616859

0.2500657030223365

0.0000000000000000

0.2380517358512945

0.4864940338034922

0.7500657030223365

0.5000000000000000

0.7422069767882843
0.9710923562377047

0.0000000000000000

0.4456131153015811

0.2500657030223365

0.0109744683101973

0.0000000000000000

0.2513566690332424

0.2500657030223365

0.0019495485285728

0.0000000000000000

0.3763467198473375

0.2500657030223365

0.0056752350460075

0.5000000000000000

0.7493912688919828

0.7500657030223365

0.5011677416703987

0.5000000000000000

0.7590970682439891

0.7500657030223365

0.5060816258763279

0.5000000000000000

0.7493888770274011

0.7500657030223365

0.4638077099380590

0.5000000000000000

0.7168581462365998

0.7500657030223365

0.4994727439654486

0.1304194708514886

0.0938274389342423

0.0575734406943011

0.3887258462792099

0.5738166977439142

0.9835718080618854

0.2500657030223365

0.2500657030223365

0.0013503024911155

0.9490000047301909

0.2500657030223365

0.2500657030223365

0.0010975152334593

0.5333546334455239

0.7500657030223365

0.7500657030223365

0.5011897881523203

0.4990043156162471

0.7500657030223365

0.7500657030223365

0.4965682488731708
0.3145033692979186

0.1441744226128918

0.4087394596428854

0.0394260599793199

0.2493008341231170

0.0000000000000000

0.2107635898350539

0.1047483626335719

0.3155917969465986

0.1441744226128918

0.4032458283975053

0.0394260599793199

0.2471656718874160

0.0000000000000000

0.2110472090820155

0.1047483626335719

0.3144053422240213

0.1441744226128918

0.3569584940458336

0.0394260599793199

0.2490918808573031

0.0000000000000000

0.2122857479928072

0.1047483626335719

0.3212229397126151

0.1441744226128918

0.3593175325157328

0.0394260599793199

0.2523926499361987

0.3926752969176712

0.4743379520572033

0.4014603208153944

0.5055639018988910

0.5792853074331309

0.3336610780559772

0.1244398483281657

0.0197345742847261

0.2300304720523672

0.3357042121360994

0.1244398483281657

0.0197345742847261

0.2302448841011727

0.3368186887518334

0.1244398483281657

0.0197345742847261

0.2305120234698437

0.3367157089886639

0.1244398483281657

0.0197345742847261

0.2303335653741686
T T T

F $\quad F \quad F$

T T T

F $\quad F \quad F$

T $T$ T

F $F \quad F$

T T $T$

F $F \quad F$

T $\mathrm{T}$ T

F $F \quad F$

$\begin{array}{lll}T & T & T\end{array}$

F $F \quad F$

$\begin{array}{lll}T & T & T\end{array}$

F $\quad F \quad F$

T $T$ T

F $F \quad F$

T $T$ T

F $\quad F \quad F$

T $T$ T

$\begin{array}{lll}\text { F } & \text { F } & \text { F }\end{array}$

$\begin{array}{lll}T & T & T\end{array}$

F $F \quad F$

T T $T$

$\begin{array}{lll}\text { F } & \text { F } & \text { F }\end{array}$

$\begin{array}{lll}T & T & T\end{array}$

$\begin{array}{lll}\text { F } & F & F\end{array}$

T $T$ T

F $F \quad F$

T T $T$

T $T$ T

$\begin{array}{lll}\mathrm{T} & \mathrm{T} & \mathrm{T}\end{array}$

T $T$ T

T $T$ T

T T $T$

T T $T$

F $F \quad F$

F $F \quad F$

T $T$ T

T T T

F $F \quad F$

F $F \quad F$

T $T$ T

T $T$ T

F $\quad F \quad F$

$\begin{array}{lll}F & F & F\end{array}$

T $T$ T

T T T

F $F \quad F$

F $F \quad F$

T $T$ T

10

\section{$\begin{array}{lllll}\text { H } & \text { C } & \text { V } & \text { O } & \text { Ti }\end{array}$ \\ $\begin{array}{lllll}7 & 2 & 1 & 37 & 16\end{array}$}

$\begin{array}{lll}0.4101405047036961 & 0.0630502624153099\end{array}$

0.2443651095075493

0.0871569321690049

0.4272054416445304

T $\quad \mathrm{T} \quad \mathrm{T}$

0.5001458684342529 T $\quad T \quad T$ 
$-0.0170151023786689$

0.9152624309553735

0.3422311544198593

0.2205491286779484

0.2233191910183428

0.0322301907527764

0.2238270378656501

0.1844854327144673

0.0000000000000000

0.2506614323504993

0.0000000000000000

0.2624142389851020

0.2500657030223364

0.0086729440554947

0.2500657030223364

0.0052317525652326

0.4999999999999841

0.7449516585360462

0.4999999999999841

0.7524290655972150

0.7500657030223350

0.3834190966063214

0.7500657030223350

0.5047389684118250

0.0000000000000000

0.2465988172694240

0.0000000000000000

0.2348760735412969

0.2500657030223364

0.9824027974402183

0.2500657030223364

0.9864034710268867

0.4999999999999841

0.7469895419940709

0.4999999999999841

0.7391140337375867

0.7500657030223350

0.5109630074382052

0.7500657030223350

0.4930088598052442

0.0508851075635048

0.2257084733704214

0.5168609530836269

0.1673509375491035

0.0673255590811795

0.0088061215379109

0.2500657030223364

0.0000000000000000

0.2594171372856249

0.5105969119614739

0.7500657030223350

0.4999999999999841

0.7510249673440844

0.9859199383512894

0.2500657030223364

0.0000000000000000

0.2368726340752014
0.5440174824066714

0.4177138866327356

0.6406753892365464

0.8266041382987109

0.7765040200836947

0.4927390877159019

0.7212353517156425

0.3142822392578204

0.0000000000000000

0.2519977656689288

0.2500657030223364

0.9712781392419582

0.0000000000000000

0.4831512086643598

0.2500657030223364

0.0140043856899888

0.0000000000000000

0.2514524303435667

0.2500657030223364

0.0027420784448944

0.0000000000000000

0.3902137121871183

0.2500657030223364

0.0026261169372525

0.4999999999999841

0.7494623686862479

0.7500657030223350

0.5065430938789428

0.4999999999999841

0.7603162996281145

0.7500657030223350

0.5040177064192729

0.4999999999999841

0.7492576237318110

0.7500657030223350

0.4661305969876995

0.4999999999999841

0.7225118430006120

0.7500657030223350

0.5007658260451167

0.1984856846883130

0.0818143823529088

0.0635600638035834

0.4025389599632034

0.6192729719866805

0.9977351502873812

0.2500657030223364

0.2500657030223364

0.0002817664248536

0.9506507607584878

0.2500657030223364

0.2500657030223364

0.0003680688333080

0.5317565600441692

0.7500657030223350

0.7500657030223350

0.5005693094409446
0.4634396917348455

0.5395689218527838

0.5627532500678729

0.5888680156154672

0.5128760886073321

0.5264295776822273

0.5567170457656939

0.4300321053756650

0.0000000000000000

0.2117643181968124

0.1047483626335719

0.3143690502564870

0.1441744226128918

0.4201780936206712

0.0394260599793199

0.2477621475501379

0.0000000000000000

0.2106643957117094

0.1047483626335719

0.3147339386253349

0.1441744226128918

0.4049727299728114

0.0394260599793199

0.2459225472299533

0.0000000000000000

0.2108937548823244

0.1047483626335719

0.3147808302407568

0.1441744226128918

0.3520949145333052

0.0394260599793199

0.2499018876950141

0.0000000000000000

0.2120598250988809

0.1047483626335719

0.3219630099513113

0.1441744226128918

0.3595062066850875

0.0394260599793199

0.2524955577554702

0.3696198747798776

0.4586636429338375

0.4006970304637719

0.5040812308273579

0.5666034459450394

0.3301570552676460

0.1244398483281657

0.0197345742847261

0.2294239557852510

0.3342032293675074

0.1244398483281657

0.0197345742847261

0.2294889782275019

0.3384047421467076

0.1244398483281657

0.0197345742847261

0.2312068895862872
T $\mathrm{T} T$

$\begin{array}{lll}T & T & T\end{array}$

$\mathrm{T} \quad \mathrm{T} \quad \mathrm{T}$

T $\mathrm{T} T$

T $\quad \mathrm{T} \quad \mathrm{T}$

$\begin{array}{lll}\mathrm{T} & \mathrm{T} & \mathrm{T}\end{array}$

$\begin{array}{lll}T & T & T\end{array}$

T $T$ T

$\begin{array}{lll}\text { F } & \text { F } & \text { F }\end{array}$

T $T$ T

F $F \quad F$

T $T$ T

F $F \quad F$

T $T$ T

F $F \quad F$

T T T

F $F \quad F$

T $T$ T

F $\quad F \quad F$

T $T$ T

F F F

T $T$ T

F $F \quad F$

T T $T$

F $\quad F \quad F$

T $T$ T

$\begin{array}{lll}\text { F } & F & \text { F }\end{array}$

T $T$ T

F $\quad F \quad F$

T T $T$

F $\quad F \quad F$

T $T$ T

$\begin{array}{lll}\text { F } & F & F\end{array}$

T $T$ T

F $F \quad F$

T $T$ T

$\begin{array}{lll}\text { F } & F & \text { F }\end{array}$

T T T

F $F \quad$ F

T T T

T $T$ T

T $T$ T

T $T$ T

T T $T$

T T T

T T T

F $F$ F

$\begin{array}{lll}F & F & F\end{array}$

T T T

T $T$ T

F $F \quad F$

F $F \quad F$

T $T$ T

T $T$ T

F $F \quad F$

F $F \quad F$

T T T 
0.4884647742763542

0.7500657030223350

0.4999999999999841

0.7421834643136251
0.5035371449763099

0.7500657030223350

0.7500657030223350

0.4963562030389790
0.3372256169683162

0.1244398483281657

0.0197345742847261

0.2309043394745528

$\begin{array}{lll}\mathrm{T} & \mathrm{T} & \mathrm{T} \\ \mathrm{F} & \mathrm{F} & \mathrm{F} \\ \mathrm{F} & \mathrm{F} & \mathrm{F} \\ \mathrm{T} & \mathrm{T} & \mathrm{T}\end{array}$

11 $\begin{array}{rrrrr}\mathbf{H} & \mathbf{C} & \mathbf{V} & \mathbf{O} & \mathbf{T i} \\ \mathbf{7} & \mathbf{2} & \mathbf{1} & \mathbf{3 7} & \mathbf{1 6}\end{array}$

0.4040576408207885 0.2325039820191515 0.0237183103726576 0.0482653767276485 0.5109384886831659 0.4500803875724070 0.3856426253436890 0.1755987330873869 0.4100162647864842 0.1989560609118885 0.0000000000000000 0.2515470489977653 0.0000000000000000 0.2657146423855686 0.2500657030223365 0.0127179400251653 0.2500657030223365 0.0064232609218260 0.5000000000000000 0.7500077034539550 0.5000000000000000 0.7515155272615121 0.7500657030223365 0.4011840614847313 0.7500657030223365 0.5067272658715379 0.0000000000000000 0.2499448205097834 0.0000000000000000 0.2398783704941427 0.2500657030223365 0.9857919112080609 0.2500657030223365 0.9914439130336296 0.5000000000000000 0.7507090570331383 0.5000000000000000 0.7450916145919891 0.7500657030223365 0.5186693571058892 0.7500657030223365 0.4970909554065466 0.0526039851967884 0.2033413314364454 0.5134062575677204 0.2469748334972468 0.2485223730090474 0.0087258672124508
0.0503729304693592 0.1181465228774976 0.6060227594377192 0.4533812264119337 0.5984760587665579 0.7949334331689887 0.7654249907094136 0.4975455136550438 0.6998992570579634 0.3214831680358548 0.0000000000000000 0.2504862220289783 0.2500657030223365 0.9838612664822883 0.0000000000000000 0.4967111093960079 0.2500657030223365 0.0110328557493731 0.0000000000000000 0.2499150457238840 0.2500657030223365 0.0155919283953435 0.0000000000000000 0.3663966809331730 0.2500657030223365 $-0.0005923004620004$ 0.5000000000000000 0.7506751061747148 0.7500657030223365 0.5182555096611116 0.5000000000000000 0.7634695787710589 0.7500657030223365 0.5028143387419395 0.5000000000000000 0.7498470588345109 0.7500657030223365 0.4672141903395281 0.5000000000000000 0.7233666468172292 0.7500657030223365 0.4955005924362427 0.2197330953952720 0.1024871518917910 0.0317721491377796 0.4392405295391263 0.6228239207217984 0.0054551779692341
0.4339277946976554 0.5049192899824627 0.4431640468736233 0.5609944968115494 0.5495571159787889 0.5881870187888156 0.5139549513487046 0.5443652997641347 0.5553647368132264 0.4253320118450128 0.0000000000000000 0.2119515075809831 0.1047483626335719 0.3170216669085236 0.1441744226128918 0.4214715832462749 0.0394260599793199 0.2493876726127345 0.0000000000000000 0.2106507615486606 0.1047483626335719 0.3174638491071207 0.1441744226128918 0.3956285015814252 0.0394260599793199 0.2470665352587809 0.0000000000000000 0.2111327822466409 0.1047483626335719 0.3134899052710411 0.1441744226128918 0.3507005755551708 0.0394260599793199 0.2484868442516440 0.0000000000000000 0.2124585753843722 0.1047483626335719 0.3199324567784281 0.1441744226128918 0.3554315068844420 0.0394260599793199 0.2493056882969811 0.3671294435837363 0.4642752763844782 0.4109866304275844 0.4994876119362474 0.5771891177247124 0.3303349492757752

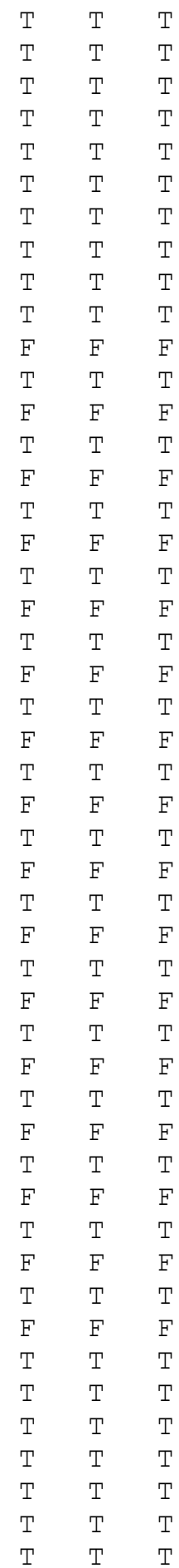


0.2500657030223365

0.0000000000000000

0.2626742487908778

0.5121246192998611

0.7500657030223365

0.5000000000000000

0.7501515042881943

0.9924389006345462

0.2500657030223365

0.0000000000000000

0.2405903834045679

0.4965692713187765

0.7500657030223365

0.5000000000000000

0.7470552452132546
0.2500657030223365

0.2500657030223365

0.9994612111616359

0.9560083465320166

0.2500657030223365

0.2500657030223365

0.0007028520390047

0.5330894142104775

0.7500657030223365

0.7500657030223365

0.4999185699405342

0.5042040556723262

0.7500657030223365

0.7500657030223365

0.4965891032157191
0.1244398483281657

0.0197345742847261

0.2298273516910235

0.3378468793115041

0.1244398483281657

0.0197345742847261

0.2296252572450739

0.3351132350417593

0.1244398483281657

0.0197345742847261

0.2297120211597894

0.3323062354602492

0.1244398483281657

0.0197345742847261

0.2292191100130782

$\begin{array}{lll}\mathrm{F} & \mathrm{F} & \mathrm{F} \\ \mathrm{F} & \mathrm{F} & \mathrm{F} \\ \mathrm{T} & \mathrm{T} & \mathrm{T} \\ \mathrm{T} & \mathrm{T} & \mathrm{T} \\ \mathrm{F} & \mathrm{F} & \mathrm{F} \\ \mathrm{F} & \mathrm{F} & \mathrm{F} \\ \mathrm{T} & \mathrm{T} & \mathrm{T} \\ \mathrm{T} & \mathrm{T} & \mathrm{T} \\ \mathrm{F} & \mathrm{F} & \mathrm{F} \\ \mathrm{F} & \mathrm{F} & \mathrm{F} \\ \mathrm{T} & \mathrm{T} & \mathrm{T} \\ \mathrm{T} & \mathrm{T} & \mathrm{T} \\ \mathrm{F} & \mathrm{F} & \mathrm{F} \\ \mathrm{F} & \mathrm{F} & \mathrm{F} \\ \mathrm{T} & \mathrm{T} & \mathrm{T}\end{array}$

\section{2}

\section{$\begin{array}{lllll}\text { H } & \text { V } & \text { O } & \text { Ti }\end{array}$}

\section{$\begin{array}{llll}3 & 1 & 35 & 16\end{array}$}

0.4037901230072900

0.2140089699687552

0.9578219926316884

0.2582303689400527

0.0000000000000000

0.2506263621371287

0.0000000000000000

0.2544923235412538

0.2500657030223365

0.0604571672222477

0.2500657030223365

0.9974961584658349

0.5000000000000000

0.7498911067349887

0.5000000000000000

0.7492005490405599

0.7500657030223365

0.4294546895717496

0.7500657030223365

0.5063008967348146

0.0000000000000000

0.2504459566173305

0.0000000000000000

0.2449713540999031

0.2500657030223365

0.9762790114849892

0.2500657030223365

0.9973568601358164

0.5000000000000000

0.7507070451519031

0.5000000000000000

0.7549601112814470

0.7500657030223365

0.5178255578023870

0.7500657030223365

0.5015478020485059

0.0700707791999650
0.9219894010271943

0.3988066008365162

0.3524974696073644

0.2201049396169380

0.0000000000000000

0.2493116693949878

0.2500657030223365

0.0048921540243336

0.0000000000000000

0.4071312377564151

0.2500657030223365

0.0000382401420171

0.0000000000000000

0.2483439572583737

0.2500657030223365

0.0310510144804887

0.0000000000000000

0.3202576381164404

0.2500657030223365

0.9924975174604368

0.5000000000000000

0.7520088973010374

0.7500657030223365

0.5367161674039038

0.5000000000000000

0.7541164140771458

0.7500657030223365

0.4989954656010395

0.5000000000000000

0.7496105041690890

0.7500657030223365

0.4772327919975044

0.5000000000000000

0.7423607554226921

0.7500657030223365

0.4931401161080900

0.0795230126616312
0.4419837688091348

0.5023540307864025

0.4190146584789392

0.4178594330459969

0.0000000000000000

0.2124824340526153

0.1047483626335719

0.3161457772479131

0.1441744226128918

0.4010531642808540

0.0394260599793199

0.2516777966504773

0.0000000000000000

0.2110238846255960

0.1047483626335719

0.3249177133572489

0.1441744226128918

0.3724024437772624

0.0394260599793199

0.2519888645582380

0.0000000000000000

0.2111836410225180

0.1047483626335719

0.3122833963064962

0.1441744226128918

0.3567905428752459

0.0394260599793199

0.2466350760058273

0.0000000000000000

0.2126701207488276

0.1047483626335719

0.3191058507645722

0.1441744226128918

0.3550903223091363

0.0394260599793199

0.2476614385983398

0.4064087875400064

$\begin{array}{lll}\text { T } & \text { T } & \text { T } \\ \text { T } & \text { T } & \text { T } \\ \text { T } & \text { T } & \text { T } \\ \text { T } & \text { T } & \text { T } \\ \text { F } & \text { F } & \text { F } \\ \text { T } & \text { T } & \text { T } \\ \text { F } & \text { F } & \text { F } \\ \text { T } & \text { T } & \text { T } \\ \text { F } & \text { F } & \text { F } \\ \text { T } & \text { T } & \text { T } \\ \text { F } & \text { F } & \text { F } \\ \text { T } & \text { T } & \text { T } \\ \text { F } & \text { F } & \text { F } \\ \text { T } & \text { T } & \text { T } \\ \text { F } & \text { F } & \text { F } \\ \text { T } & \text { T } & \text { T } \\ \text { F } & \text { F } & \text { F } \\ \text { T } & \text { T } & \text { T } \\ \text { F } & \text { F } & \text { F } \\ \text { T } & \text { T } & \text { T } \\ \text { F } & \text { F } & \text { F } \\ \text { T } & \text { T } & \text { T } \\ \text { F } & \text { F } & \text { F } \\ \text { T } & \text { T } & \text { T } \\ \text { F } & \text { F } & \text { F } \\ \text { T } & \text { T } & \text { T } \\ \text { F } & \text { F } & \text { F } \\ \text { T } & \text { T } & \text { T } \\ \text { F } & \text { F } & \text { F } \\ \text { T } & \text { T } & \text { T } \\ \text { F } & \text { F } & \text { F } \\ \text { T } & \text { T } & \text { T } \\ \text { F } & \text { F } & \text { F } \\ \text { T } & \text { T } & \text { T } \\ \text { F } & \text { F } & \text { F } \\ \text { T } & \text { T } & \text { T } \\ \text { T } & \text { T } & \text { T } \\ & & \text { T }\end{array}$


0.2770819900212241

0.4332237249247493

0.9976325570789866

0.2500657030223365

0.0000000000000000

0.2519795082828753

0.5062130700601487

0.7500657030223365

0.5000000000000000

0.7518449869217683

$-0.0004738102492022$

0.2500657030223365

0.0000000000000000

0.2476288216140635

0.4996580584815442

0.7500657030223365

0.5000000000000000

0.7517882372790605
0.2924365917799577

0.0266775479137096

0.9813368072119659

0.2500657030223365

0.2500657030223365

0.0001382579829614

0.9695615253243232

0.2500657030223365

0.2500657030223365

0.0009963223419970

0.5350939496543144

0.7500657030223365

0.7500657030223365

0.5009191233523373

0.5100242633550187

0.7500657030223365

0.7500657030223365

0.4969929195169429
0.4910514957271545

0.4198549406747053

0.3375032169153308

0.1244398483281657

0.0197345742847261

0.2312068613691952

0.3372317486636779

0.1244398483281657

0.0197345742847261

0.2293854354967362

0.3307323811700764

0.1244398483281657

0.0197345742847261

0.2282328253793654

0.3293141856257367

0.1244398483281657

0.0197345742847261

0.2280454285526596

$\begin{array}{lll}\mathrm{T} & \mathrm{T} & \mathrm{T} \\ \mathrm{T} & \mathrm{T} & \mathrm{T} \\ \mathrm{T} & \mathrm{T} & \mathrm{T} \\ \mathrm{F} & \mathrm{F} & \mathrm{F} \\ \mathrm{F} & \mathrm{F} & \mathrm{F} \\ \mathrm{T} & \mathrm{T} & \mathrm{T} \\ \mathrm{T} & \mathrm{T} & \mathrm{T} \\ \mathrm{F} & \mathrm{F} & \mathrm{F} \\ \mathrm{F} & \mathrm{F} & \mathrm{F} \\ \mathrm{T} & \mathrm{T} & \mathrm{T} \\ \mathrm{T} & \mathrm{T} & \mathrm{T} \\ \mathrm{F} & \mathrm{F} & \mathrm{F} \\ \mathrm{F} & \mathrm{F} & \mathrm{F} \\ \mathrm{T} & \mathrm{T} & \mathrm{T} \\ \mathrm{T} & \mathrm{T} & \mathrm{T} \\ \mathrm{F} & \mathrm{F} & \mathrm{F} \\ \mathrm{F} & \mathrm{F} & \mathrm{F} \\ \mathrm{T} & \mathrm{T} & \mathrm{T}\end{array}$

13

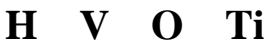 \\ $\begin{array}{llll}3 & 1 & 35 & 16\end{array}$}

0.3452402883731875

0.1329712984516666

0.4549358170531872

0.2559547887743091

0.0000000000000000

0.2502457927283995

0.0000000000000000

0.2493001408830109

0.2500657030223364

0.0654888472445224

0.2500657030223364

0.9995486343327686

0.4999999999999942

0.7485581278786525

0.4999999999999942

0.7500602729053294

0.7500657030223350

0.4379731072536865

0.7500657030223350

0.5014957421873992

0.0000000000000000

0.2482296195212627

0.0000000000000000

0.2467067420044683

0.2500657030223364

0.9758258347653829

0.2500657030223364

0.9978524561434194

0.4999999999999942

0.7510311098616340

0.4999999999999942

0.7498194537121120

0.7500657030223350

0.5046493899246504
0.3872424352410331 0.3966806348387549 0.9512152905326964 0.1747347127323206 0.0000000000000000 0.2482084800865552 0.2500657030223364 0.0359406714573385 0.0000000000000000 0.3793434972559299 0.2500657030223364 0.9897214015748618 0.0000000000000000 0.2459017732750283 0.2500657030223364 0.0286220960593205 0.0000000000000000 0.3119241637607889 0.2500657030223364 0.9906779937996606 0.4999999999999942 0.7530864168707990 0.7500657030223350 0.5485932566854377 0.4999999999999942 0.7449575415072849 0.7500657030223350 0.4878798996334933 0.4999999999999942 0.7495866009071668 0.7500657030223350 0.4861185329625801 0.4999999999999942 0.7460139853303525
0.4806775158880209 0.4351756014331323 0.4575227563246459 0.3996016597249348 0.0000000000000000 0.2117064800674568 0.1047483626335719 0.3200814781191635 0.1441744226128918 0.3898519435821906 0.0394260599793199 0.2524890145001089 0.0000000000000000 0.2111606647532943 0.1047483626335719 0.3237750819225119 0.1441744226128918 0.3640269775101208 0.0394260599793199 0.2522863225822232 0.0000000000000000 0.2104119999934052 0.1047483626335719 0.3094539661857035 0.1441744226128918 0.3567705126592007 0.0394260599793199 0.2461101433369251 0.0000000000000000 0.2123886736968260 0.1047483626335719 0.3158350598448578 0.1441744226128918 0.3562037655277946

$\begin{array}{lll}\mathrm{T} & \mathrm{T} & \mathrm{T} \\ \mathrm{T} & \mathrm{T} & \mathrm{T} \\ \mathrm{T} & \mathrm{T} & \mathrm{T} \\ \mathrm{T} & \mathrm{T} & \mathrm{T} \\ \mathrm{F} & \mathrm{F} & \mathrm{F} \\ \mathrm{T} & \mathrm{T} & \mathrm{T} \\ \mathrm{F} & \mathrm{F} & \mathrm{F} \\ \mathrm{T} & \mathrm{T} & \mathrm{T} \\ \mathrm{F} & \mathrm{F} & \mathrm{F} \\ \mathrm{T} & \mathrm{T} & \mathrm{T} \\ \mathrm{F} & \mathrm{F} & \mathrm{F} \\ \mathrm{T} & \mathrm{T} & \mathrm{T} \\ \mathrm{F} & \mathrm{F} & \mathrm{F} \\ \mathrm{T} & \mathrm{T} & \mathrm{T} \\ \mathrm{F} & \mathrm{F} & \mathrm{F} \\ \mathrm{T} & \mathrm{T} & \mathrm{T} \\ \mathrm{F} & \mathrm{F} & \mathrm{F} \\ \mathrm{T} & \mathrm{T} & \mathrm{T} \\ \mathrm{F} & \mathrm{F} & \mathrm{F} \\ \mathrm{T} & \mathrm{T} & \mathrm{T} \\ \mathrm{F} & \mathrm{F} & \mathrm{F} \\ \mathrm{T} & \mathrm{T} & \mathrm{T} \\ \mathrm{F} & \mathrm{F} & \mathrm{F} \\ \mathrm{T} & \mathrm{T} & \mathrm{T} \\ \mathrm{F} & \mathrm{F} & \mathrm{F} \\ \mathrm{T} & \mathrm{T} & \mathrm{T} \\ \mathrm{F} & \mathrm{F} & \mathrm{F} \\ \mathrm{T} & \mathrm{T} & \mathrm{T} \\ \mathrm{F} & \mathrm{F} & \mathrm{F} \\ \mathrm{T} & \mathrm{T} & \mathrm{T} \\ \mathrm{F} & \mathrm{F} & \mathrm{F} \\ \mathrm{T} & \mathrm{T} & \mathrm{T} \\ \mathrm{F} & \mathrm{F} & \mathrm{F} \\ \mathrm{T} & \mathrm{T} & \mathrm{T} \\ & & \end{array}$


0.7500657030223350

0.5001009759691777

0.0714960513531567

0.2398174847250021

0.4597522593347984

0.9879936587354483

0.2500657030223364

0.0000000000000000

0.2528995218053957

0.4994483064796170

0.7500657030223350

0.4999999999999942

0.7477023090870089

0.0013945025776252

0.2500657030223364

0.0000000000000000

0.2461502967211911

0.4973851025003975

0.7500657030223350

0.4999999999999942

0.7511445805440427
0.7500657030223350

0.4942699968774434

0.0423775077727102

0.3235762611777653

0.0319897724315723

0.9712176463281482

0.2500657030223364

0.2500657030223364

0.0009773389367533

0.9771092959406682

0.2500657030223364

0.2500657030223364

0.9992378586382310

0.5227048799326510

0.7500657030223350

0.7500657030223350

0.5010397680491118

0.5142630956740192

0.7500657030223350

0.7500657030223350

0.4958460836798722
0.0394260599793199

0.2451826213826015

0.4110661777333104

0.4695385102427609

0.4251912511151560

0.3376631291800541

0.1244398483281657

0.0197345742847261

0.2307586443793470

0.3413286002411056

0.1244398483281657

0.0197345742847261

0.2307319590178977

0.3312085118980943

0.1244398483281657

0.0197345742847261

0.2280335613771839

0.3273865839663207

0.1244398483281657

0.0197345742847261

0.2286339581955695

$\begin{array}{lll}\mathrm{F} & \mathrm{F} & \mathrm{F} \\ \mathrm{T} & \mathrm{T} & \mathrm{T} \\ \mathrm{T} & \mathrm{T} & \mathrm{T} \\ \mathrm{T} & \mathrm{T} & \mathrm{T} \\ \mathrm{T} & \mathrm{T} & \mathrm{T} \\ \mathrm{T} & \mathrm{T} & \mathrm{T} \\ \mathrm{F} & \mathrm{F} & \mathrm{F} \\ \mathrm{F} & \mathrm{F} & \mathrm{F} \\ \mathrm{T} & \mathrm{T} & \mathrm{T} \\ \mathrm{T} & \mathrm{T} & \mathrm{T} \\ \mathrm{F} & \mathrm{F} & \mathrm{F} \\ \mathrm{F} & \mathrm{F} & \mathrm{F} \\ \mathrm{T} & \mathrm{T} & \mathrm{T} \\ \mathrm{T} & \mathrm{T} & \mathrm{T} \\ \mathrm{F} & \mathrm{F} & \mathrm{F} \\ \mathrm{F} & \mathrm{F} & \mathrm{F} \\ \mathrm{T} & \mathrm{T} & \mathrm{T} \\ \mathrm{T} & \mathrm{T} & \mathrm{T} \\ \mathrm{F} & \mathrm{F} & \mathrm{F} \\ \mathrm{F} & \mathrm{F} & \mathrm{F} \\ \mathrm{T} & \mathrm{T} & \mathrm{T}\end{array}$

\section{4}

\section{$\begin{array}{lllll}H & \text { V } & \text { O } & \text { Ti }\end{array}$ \\ $\begin{array}{llll}3 & 1 & 35 & 16\end{array}$}

0.4419888535892367

0.1677381992162335

0.3653400591260640

0.2463262497679276

0.0000000000000000

0.2487902001079346

0.0000000000000000

0.2500133622773476

0.2500657030223365

0.0677324184480274

0.2500657030223365

0.0010336282218677

0.5000000000000000

0.7511981228511667

0.5000000000000000

0.7515218196662141

0.7500657030223365

0.4390649402114344

0.7500657030223365

0.5014953018510537

0.0000000000000000

0.2517573349095408

0.0000000000000000

0.2491502506714435

0.2500657030223365

0.9815097574822401

0.2500657030223365

$-0.0012369689121486$

0.5000000000000000

0.7490622480020237

0.5000000000000000
0.9436995965660645

0.4171781730189850

0.3705071179378009

0.1796263379674284

0.0000000000000000

0.2477653467348348

0.2500657030223365

0.0413376715362052

0.0000000000000000

0.3531079967474206

0.2500657030223365

0.9848360002115240

0.0000000000000000

0.2449397103121303

0.2500657030223365

0.0266091310816523

0.0000000000000000

0.3273203298683201

0.2500657030223365

0.9929873390504048

0.5000000000000000

0.7537702058406582

0.7500657030223365

0.5562822783419941

0.5000000000000000

0.7448311768758754

0.7500657030223365

0.4894025113185587

0.5000000000000000

0.7500929599374518

0.7500657030223365
0.4506291188462077

0.4676483466003505

0.4810491033685549

0.3959672436426899

0.0000000000000000

0.2116689088241685

0.1047483626335719

0.3214739737882146

0.1441744226128918

0.3776700542997280

0.0394260599793199

0.2540874042590255

0.0000000000000000

0.2114860312436488

0.1047483626335719

0.3251349877787812

0.1441744226128918

0.3692360518339635

0.0394260599793199

0.2533975876176938

0.0000000000000000

0.2100556886721888

0.1047483626335719

0.3086686719221026

0.1441744226128918

0.3588586607153380

0.0394260599793199

0.2449320680922109

0.0000000000000000

0.2123640704937189

0.1047483626335719

\section{$\mathrm{T}$}

\section{$\mathrm{T}$}

$T$

$\begin{array}{lll}T & T\end{array}$

$\begin{array}{lll}\text { F } & F & F\end{array}$

T $T$ T

F $F \quad F$

T $T$ T

F $F \quad F$

T $T$ T

F $F \quad F$

T T $T$

F F F

T $T$ T

F $F \quad$ F

T $T$ T

F $F \quad$ F

T $T$ T

F $F \quad F$

T $T$ T

F F F

T T T

F $F \quad$ F

T $T$ T

F $F \quad F$

T $T$ T

F $F \quad F$

T $T$ T

F $\quad F \quad F$

$\mathrm{T} T \mathrm{~T}$

F F F 
0.7484431026421013

0.7500657030223365

0.5036221539970782

0.7500657030223365

0.5000439217452731

0.0818272299337744

0.2483858192980904

0.4644499777558426

0.9899854081540839

0.2500657030223365

0.0000000000000000

0.2516284382828899

0.5028529764708544

0.7500657030223365

0.5000000000000000

0.7487861426113785

0.0025346903554093

0.2500657030223365

0.0000000000000000

0.2484602021018942

0.4963448347313699

0.7500657030223365

0.5000000000000000

0.7504121787403690
0.4937471303408081

0.5000000000000000

0.7490481095372618

0.7500657030223365

0.4905746040834961

0.0342407279772467

0.3201464295839260

0.0425917343840026

0.9689641012984366

0.2500657030223365

0.2500657030223365

0.0014334291470035

0.9780344037687998

0.2500657030223365

0.2500657030223365

0.9981802850904005

0.5195516269824291

0.7500657030223365

0.7500657030223365

0.5018409819861193

0.5196512222447310

0.7500657030223365

0.7500657030223365

0.4952395907158895
0.3152557489710220

0.1441744226128918

0.3588513093381612

0.0394260599793199

0.2451293767949868

0.4158016224907372

0.4761841126913334

0.4251173902534676

0.3400831569908213

0.1244398483281657

0.0197345742847261

0.2306121268971146

0.3420131859986800

0.1244398483281657

0.0197345742847261

0.2312388002858506

0.3291435209834634

0.1244398483281657

0.0197345742847261

0.2277531259558165

0.3279107277866697

0.1244398483281657

0.0197345742847261

0.2286157968315810

$\begin{array}{lll}\mathrm{T} & \mathrm{T} & \mathrm{T} \\ \mathrm{F} & \mathrm{F} & \mathrm{F} \\ \mathrm{T} & \mathrm{T} & \mathrm{T} \\ \mathrm{F} & \mathrm{F} & \mathrm{F} \\ \mathrm{T} & \mathrm{T} & \mathrm{T} \\ \mathrm{T} & \mathrm{T} & \mathrm{T} \\ \mathrm{T} & \mathrm{T} & \mathrm{T} \\ \mathrm{T} & \mathrm{T} & \mathrm{T} \\ \mathrm{T} & \mathrm{T} & \mathrm{T} \\ \mathrm{F} & \mathrm{F} & \mathrm{F} \\ \mathrm{F} & \mathrm{F} & \mathrm{F} \\ \mathrm{T} & \mathrm{T} & \mathrm{T} \\ \mathrm{T} & \mathrm{T} & \mathrm{T} \\ \mathrm{F} & \mathrm{F} & \mathrm{F} \\ \mathrm{F} & \mathrm{F} & \mathrm{F} \\ \mathrm{T} & \mathrm{T} & \mathrm{T} \\ \mathrm{T} & \mathrm{T} & \mathrm{T} \\ \mathrm{F} & \mathrm{F} & \mathrm{F} \\ \mathrm{F} & \mathrm{F} & \mathrm{F} \\ \mathrm{T} & \mathrm{T} & \mathrm{T} \\ \mathrm{T} & \mathrm{T} & \mathrm{T} \\ \mathrm{F} & \mathrm{F} & \mathrm{F} \\ \mathrm{F} & \mathrm{F} & \mathrm{F} \\ \mathrm{T} & \mathrm{T} & \mathrm{T}\end{array}$

15

\section{$\begin{array}{lllll}H & \text { V } & \text { O } & \text { Ti }\end{array}$ \\ $\begin{array}{llll}1 & 1 & 34 & 16\end{array}$}

0.5528774295612960

0.2456941987420807

0.0000000000000000

0.2441485083987941

0.0000000000000000

0.2564612667054941

0.2500657030223365

0.0587185860168662

0.2500657030223365

0.0027204841391305

0.5000000000000000

0.7517287256031240

0.5000000000000000

0.7515114798626619

0.7500657030223365

0.4156255698826627

0.7500657030223365

0.5021474169886239

0.0000000000000000

0.2525908661344324

0.0000000000000000

0.2451339475321903

0.2500657030223365

0.9821954460015563

0.2500657030223365

$-0.0054971418030491$

0.5000000000000000

0.7454377474969057
0.1504882571591668

0.1761531401901620

0.0000000000000000

0.2475532091295129

0.2500657030223365

0.0356912175355014

0.0000000000000000

0.3296533744199240

0.2500657030223365

0.9844236606947045

0.0000000000000000

0.2451728464647630

0.2500657030223365

0.0280367649646395

0.0000000000000000

0.3633103085883989

0.2500657030223365

0.9950429978255695

0.5000000000000000

0.7537111057129031

0.7500657030223365

0.5642209814127528

0.5000000000000000

0.7509861020083486

0.7500657030223365

0.4938218329314762

0.5000000000000000

0.7500723625138582
0.4355877931889698

0.3899540547111316

0.0000000000000000

0.2117072716380077

0.1047483626335719

0.3218355031850977

0.1441744226128918

0.3657941635080793

0.0394260599793199

0.2549033655450708

0.0000000000000000

0.2114079051814423

0.1047483626335719

0.3244935121572556

0.1441744226128918

0.3812402670168653

0.0394260599793199

0.2525239082376043

0.0000000000000000

0.2096651781558081

0.1047483626335719

0.3081382516696871

0.1441744226128918

0.3592067976278173

0.0394260599793199

0.2447467538986925

0.0000000000000000

0.2124963184858460

$\begin{array}{lll}\text { T } & \text { T } & \text { T } \\ \text { T } & \text { T } & \text { T } \\ \text { F } & \text { F } & \text { F } \\ \text { T } & \text { T } & \text { T } \\ \text { F } & \text { F } & \text { F } \\ \text { T } & \text { T } & \text { T } \\ \text { F } & \text { F } & \text { F } \\ \text { T } & \text { T } & \text { T } \\ \text { F } & \text { F } & \text { F } \\ \text { T } & \text { T } & \text { T } \\ \text { F } & \text { F } & \text { F } \\ \text { T } & \text { T } & \text { T } \\ \text { F } & \text { F } & \text { F } \\ \text { T } & \text { T } & \text { T } \\ \text { F } & \text { F } & \text { F } \\ \text { T } & \text { T } & \text { T } \\ \text { F } & \text { F } & \text { F } \\ \text { T } & \text { T } & \text { T } \\ \text { F } & \text { F } & \text { F } \\ \text { T } & \text { T } & \text { T } \\ \text { F } & \text { F } & \text { F } \\ \text { T } & \text { T } & \text { T } \\ \text { F } & \text { F } & \text { F } \\ \text { T } & \text { T } & \text { T } \\ \text { F } & \text { F } & \text { F } \\ \text { T } & \text { T } & \text { T } \\ \text { F } & \text { F } & \text { F } \\ \text { T } & \text { T } & \text { T } \\ & & \end{array}$


0.5000000000000000
0.7433585267420554
0.7500657030223365
0.5098161605355843
0.7500657030223365
0.4952081327261345
0.0912078587486395
0.4759483473745552
0.9901360696179481
0.2500657030223365
0.0000000000000000
0.2511370928884901
0.5071077340848542
0.7500657030223365
0.5000000000000000
0.7495505860598120
0.0036951699119600
0.2500657030223365
0.0000000000000000
0.2463109393247724
0.4879861479728777
0.7500657030223365
0.5000000000000000
0.7431494665659457
0.7500657030223365

0.4944642167352630

0.5000000000000000

0.7461971194776553

0.7500657030223365

0.4847016425673453

0.0430319943589298

0.0621566632214168

0.9748863081499708

0.2500657030223365

0.2500657030223365

0.0006045850824443

0.9695243454405477

0.2500657030223365

0.2500657030223365

$-0.0019579573995206$

0.5214477422541733

0.7500657030223365

0.7500657030223365

0.5017651857777792

0.5236847950648844

0.7500657030223365

0.7500657030223365

0.4945690641591006
0.1047483626335719

0.3156311183173017

0.1441744226128918

0.3607959830871098

0.0394260599793199

0.2463058129284145

0.4203383009407187

0.4187853530095448

0.3411096378709733

0.1244398483281657

0.0197345742847261

0.2295378005069385

0.3405713877329595

0.1244398483281657

0.0197345742847261

0.2310547058286224

0.3277421946682024

0.1244398483281657

0.0197345742847261

0.2280069741878593

0.3308858141807620

0.1244398483281657

0.0197345742847261

0.2291672813875916

$\begin{array}{lll}\text { F } & F & F \\ \text { T } & \text { T } & \text { T } \\ \text { F } & F & F \\ \text { T } & \text { T } & \text { T } \\ \text { F } & \text { F } & \text { F } \\ \text { T } & \text { T } & \text { T } \\ \text { T } & \text { T } & \text { T } \\ \text { T } & \text { T } & \text { T } \\ \text { T } & \text { T } & \text { T } \\ \text { F } & \text { F } & \text { F } \\ \text { F } & \text { F } & \text { F } \\ \text { T } & \text { T } & \text { T } \\ \text { T } & \text { T } & \text { T } \\ \text { F } & \text { F } & \text { F } \\ \text { F } & \text { F } & \text { F } \\ \text { T } & \text { T } & \text { T } \\ \text { T } & \text { T } & \text { T } \\ \text { F } & \text { F } & \text { F } \\ \text { F } & \text { F } & \text { F } \\ \text { T } & \text { T } & \text { T } \\ \text { T } & \text { T } & \text { T } \\ \text { F } & \text { F } & \text { F } \\ \text { F } & \text { F } & \text { F } \\ \text { T } & \text { T } & \text { T }\end{array}$

Table S7. Cartesian Coordinates of Stationary States Calculated with the Cluster Model.

\section{For Figure 2: 2-10}

2

$\begin{array}{lrrr}\mathrm{O} & -0.490860 & 1.647806 & -0.389881 \\ \mathrm{O} & 1.902330 & 0.495591 & -1.475042 \\ \mathrm{O} & 1.710583 & 1.050747 & 1.322338 \\ \mathrm{O} & -0.529083 & -1.022930 & -1.310871 \\ \mathrm{O} & -0.695530 & -0.483744 & 1.456310 \\ \mathrm{O} & 1.951386 & -2.339533 & -2.609858 \\ \mathrm{O} & 1.662544 & -1.666986 & 0.386332 \\ \mathrm{O} & 1.588068 & -1.185677 & 3.418719 \\ \mathrm{~V} & -1.271779 & 0.079559 & -0.143312 \\ \mathrm{O} & 2.022154 & 3.428740 & -0.617730 \\ \mathrm{O} & -2.850134 & 0.144635 & -0.257017 \\ \mathrm{TI} & 1.315581 & -1.228421 & -1.343909 \\ \mathrm{TI} & 1.096927 & -0.618914 & 1.782683 \\ \mathrm{TI} & 1.359034 & 1.785024 & -0.300248 \\ \mathrm{H} & 1.196275 & -1.568643 & 4.209778 \\ \mathrm{H} & 2.158765 & -3.271049 & -2.731547 \\ \mathrm{H} & 2.413160 & 4.166953 & -0.140634 \\ \mathrm{C} & -6.496770 & 0.503478 & -0.108075 \\ \mathrm{H} & -7.545966 & 0.218528 & 0.040228 \\ \mathrm{H} & -6.387868 & 0.911698 & -1.127476 \\ \mathrm{H} & -6.245443 & 1.295424 & 0.618318\end{array}$




$\begin{array}{lllr}\mathrm{O} & -5.715012 & -0.667790 & 0.085548 \\ \mathrm{H} & -4.782873 & -0.439712 & -0.042399\end{array}$

3

\begin{tabular}{lrrr}
\hline & -0.447240 & -1.242442 & 1.319434 \\
$\mathrm{O}$ & -0.215264 & -1.141926 & -1.431752 \\
$\mathrm{O}$ & -1.337894 & 0.915297 & -0.189833 \\
$\mathrm{O}$ & 2.160989 & -0.884945 & 0.140911 \\
$\mathrm{O}$ & 0.904104 & 1.279758 & 1.517234 \\
$\mathrm{O}$ & 2.490197 & -0.909986 & -2.933255 \\
$\mathrm{O}$ & 1.175709 & 1.340387 & -1.374785 \\
$\mathrm{O}$ & -0.229125 & 3.743921 & -0.020003 \\
$\mathrm{~V}$ & -1.377256 & -0.984780 & -0.126454 \\
$\mathrm{O}$ & -2.308198 & -2.381829 & -0.378182 \\
$\mathrm{O}$ & 2.009433 & -1.001102 & 3.202083 \\
$\mathrm{C}$ & -4.171351 & 0.537463 & -0.150974 \\
$\mathrm{H}$ & -5.183130 & 0.367072 & 0.245386 \\
$\mathrm{H}$ & -4.230812 & 0.634077 & -1.246602 \\
$\mathrm{H}$ & -3.774072 & 1.470467 & 0.267919 \\
$\mathrm{O}$ & -3.352133 & -0.553018 & 0.233354 \\
$\mathrm{H}$ & -3.262208 & -1.733932 & -0.173132 \\
$\mathrm{TI}$ & 1.506812 & -0.432126 & -1.494977 \\
$\mathrm{TI}$ & 1.227848 & -0.470415 & 1.664508 \\
$\mathrm{TI}$ & 0.078878 & 1.956492 & -0.020990 \\
$\mathrm{H}$ & -0.999069 & 4.310398 & -0.128294 \\
$\mathrm{H}$ & 2.259158 & -0.586939 & 4.034245 \\
$\mathrm{H}$ & 3.192656 & -1.535831 & -3.134770
\end{tabular}

4

$\begin{array}{lrrr}\mathrm{O} & 1.281442 & 0.696626 & -0.932264 \\ \mathrm{O} & -0.137841 & -1.437412 & -1.013550 \\ \mathrm{O} & 0.715950 & -0.938100 & 1.450012 \\ \mathrm{O} & -1.493416 & 0.941408 & -1.490527 \\ \mathrm{O} & -0.538212 & 1.656151 & 1.111791 \\ \mathrm{O} & -3.006779 & -1.769538 & -1.948674 \\ \mathrm{O} & -2.093399 & -0.709995 & 0.853908 \\ \mathrm{O} & -1.247646 & 0.084110 & 3.649042 \\ \mathrm{~V} & 1.476522 & -0.879618 & -0.100105 \\ \mathrm{O} & 2.124143 & -2.413852 & -0.798520 \\ \mathrm{O} & 0.118174 & 3.520666 & -1.266066 \\ \mathrm{C} & 4.141452 & 0.360583 & -0.163417 \\ \mathrm{H} & 4.694210 & -0.245791 & -0.899472 \\ \mathrm{H} & 4.831480 & 0.682099 & 0.629139 \\ \mathrm{H} & 3.714826 & 1.240436 & -0.672127 \\ \mathrm{O} & 3.101506 & -0.390594 & 0.414974 \\ \mathrm{H} & 3.048481 & -2.575036 & -0.562897 \\ \mathrm{TI} & -1.776718 & -0.841897 & -0.982028\end{array}$




$\begin{array}{llrr}\text { TI } & -0.840901 & 0.053780 & 1.887306 \\ \text { TI } & -0.161946 & 1.823141 & -0.694854 \\ \text { H } & -0.293703 & 4.087739 & -1.925658 \\ \text { H } & -2.953859 & -2.556976 & -2.499604 \\ \text { H } & -1.291096 & 0.748961 & 4.343188\end{array}$

\section{5}

$\begin{array}{lrrr}\mathrm{O} & 1.425560 & 0.722679 & -0.919277 \\ \mathrm{O} & -0.236837 & -1.343376 & -1.139393 \\ \mathrm{O} & 0.719250 & -1.079395 & 1.393799 \\ \mathrm{O} & -1.426022 & 1.144131 & -1.394848 \\ \mathrm{O} & -0.364939 & 1.575429 & 1.222679 \\ \mathrm{O} & -3.121559 & -1.451641 & -2.020743 \\ \mathrm{O} & -2.103068 & -0.650473 & 0.838124 \\ \mathrm{O} & -1.165061 & -0.122853 & 3.660424 \\ \mathrm{~V} & 1.445734 & -1.075975 & -0.189861 \\ \mathrm{O} & 1.948315 & -2.639143 & -0.920627 \\ \mathrm{O} & 0.288861 & 3.606948 & -0.964295 \\ \mathrm{C} & 3.806815 & 0.570336 & -0.138962 \\ \mathrm{H} & 4.496925 & 0.460047 & -0.985086 \\ \mathrm{H} & 4.156565 & 1.172799 & 0.707450 \\ \mathrm{O} & 3.107699 & -0.517746 & 0.189343 \\ \mathrm{H} & 2.877060 & -2.870568 & -0.780527 \\ \mathrm{H} & 2.483199 & 1.087710 & -0.724407 \\ \mathrm{TI} & -0.773133 & -0.067085 & 1.895703 \\ \mathrm{TI} & -1.824349 & -0.675979 & -1.006965 \\ \mathrm{TI} & -0.059313 & 1.879619 & -0.559643 \\ \mathrm{H} & -0.078647 & 4.240387 & -1.589512 \\ \mathrm{H} & -3.104551 & -2.178505 & -2.651654 \\ \mathrm{H} & -1.051452 & 0.452842 & 4.422712\end{array}$

6

0

0

O

0

0

0

0

O

V

O

O

C

$\mathrm{H}$

$\mathrm{H}$

O

$$
\begin{array}{r}
-0.986152 \\
0.336695 \\
-1.224564 \\
1.990651 \\
0.352923 \\
3.273645 \\
1.670846 \\
0.026353 \\
-1.505112 \\
-2.182595 \\
0.776668 \\
-3.875553 \\
-4.945257 \\
-3.307890 \\
-3.322917
\end{array}
$$

$$
\begin{array}{r}
-1.685024 \\
-0.245313 \\
1.316054 \\
-1.285717 \\
0.221484 \\
0.021183 \\
1.612380 \\
3.302327 \\
-0.213917 \\
-0.635771 \\
-2.726517 \\
0.170180 \\
-0.023252 \\
0.746078 \\
-0.243180
\end{array}
$$




$\begin{array}{lrrr}\mathrm{H} & -3.132953 & 2.850819 & -0.581898 \\ \mathrm{H} & -1.739743 & -0.655642 & -2.118069 \\ \mathrm{TI} & 1.912331 & 1.145332 & 0.033932 \\ \mathrm{TI} & 0.621482 & -1.332852 & -1.425537 \\ \mathrm{TI} & 0.219575 & -0.884728 & 1.733759 \\ \mathrm{H} & 3.277164 & 3.309985 & -0.149812 \\ \mathrm{H} & 0.627104 & -1.937078 & 4.039945 \\ \mathrm{H} & 0.825498 & -3.547003 & -2.756632\end{array}$

7

$\begin{array}{lrrr}0 & 0.386133 & -1.902026 & -0.821908 \\ \mathrm{O} & 1.478872 & -0.177407 & 1.180294 \\ \mathrm{O} & 1.116200 & 0.896554 & -1.474324 \\ \mathrm{O} & -1.229643 & -0.885873 & 1.444896 \\ \mathrm{O} & -1.651746 & 0.137918 & -1.167535 \\ \mathrm{O} & 0.164489 & 0.826603 & 3.696242 \\ \mathrm{O} & -0.451062 & 1.849823 & 0.771228 \\ \mathrm{O} & -1.004139 & 3.057276 & -1.972379 \\ \mathrm{~V} & 1.886092 & -0.496228 & -0.678682 \\ \mathrm{O} & 3.479975 & -1.289874 & -0.851635 \\ \mathrm{O} & -2.630127 & -2.684812 & -0.553864 \\ \mathrm{H} & 4.299924 & -1.046030 & -1.293119 \\ \mathrm{H} & 0.449244 & -2.475685 & -1.596502 \\ \mathrm{TI} & 0.040574 & 0.437864 & 1.918413 \\ \mathrm{TI} & -1.405040 & -1.386169 & -0.244819 \\ \mathrm{TI} & -0.521675 & 1.604780 & -0.994645 \\ \mathrm{H} & 0.867419 & 0.699199 & 4.340835 \\ \mathrm{H} & -1.321641 & 3.935301 & -1.738873 \\ \mathrm{H} & -3.451596 & -2.767480 & -1.047640\end{array}$

8

$\begin{array}{lrrr}0 & 2.134471 & -0.034049 & -0.348619 \\ \mathrm{O} & -0.351250 & 1.709645 & -1.292180 \\ \mathrm{O} & -0.323058 & -1.181894 & -1.741163 \\ \mathrm{O} & 0.247007 & 1.214678 & 1.573124 \\ \mathrm{O} & 0.196111 & -1.675514 & 1.118626 \\ \mathrm{O} & -2.114052 & 2.969942 & 0.834747 \\ \mathrm{O} & -2.051683 & -0.046840 & 0.332227 \\ \mathrm{O} & -2.194456 & -3.043590 & -0.175725 \\ \mathrm{~V} & 0.624531 & 0.273449 & -1.607877 \\ \mathrm{O} & 2.390577 & 0.545878 & -2.477480 \\ \mathrm{O} & 2.524154 & -0.464401 & 2.621917 \\ \mathrm{H} & 2.689131 & 0.182150 & -3.316968 \\ \mathrm{H} & 2.728507 & 0.164640 & -1.328226 \\ \mathrm{TI} & 1.321602 & -0.246541 & 1.291216 \\ \mathrm{TI} & -1.100110 & 1.540501 & 0.391051 \\ \mathrm{TI} & -1.160399 & -1.560135 & -0.101688\end{array}$




$\begin{array}{rrrr}\mathrm{H} & -3.129947 & -3.253654 & -0.251799 \\ \mathrm{H} & 2.894803 & -1.185866 & 3.138764 \\ \mathrm{H} & -2.553325 & 3.708410 & 0.402862\end{array}$

9

$\begin{array}{lrrr}\mathrm{O} & -0.922819 & 0.024003 & -1.933579 \\ \mathrm{O} & 1.122765 & -1.651487 & -0.730316 \\ \mathrm{O} & 1.321494 & 1.483843 & -0.764547 \\ \mathrm{O} & -1.477974 & -1.358720 & 0.590435 \\ \mathrm{O} & -1.307817 & 1.528360 & 0.567618 \\ \mathrm{O} & 0.676279 & -3.041096 & 1.927905 \\ \mathrm{O} & 0.839680 & -0.021448 & 1.765740 \\ \mathrm{O} & 1.053815 & 3.005757 & 1.824936 \\ \mathrm{~V} & 0.773679 & -0.073920 & -1.489675 \\ \mathrm{O} & 2.907797 & -0.168288 & -2.160739 \\ \mathrm{O} & -3.725182 & 0.237773 & -0.738915 \\ \mathrm{H} & 3.345800 & 0.588996 & -1.742006 \\ \mathrm{H} & 3.299705 & -0.968076 & -1.778425 \\ \mathrm{TI} & -1.951699 & 0.121376 & -0.388007 \\ \mathrm{TI} & 0.300321 & -1.568415 & 0.935801 \\ \mathrm{TI} & 0.505377 & 1.552436 & 0.887516 \\ \mathrm{H} & -4.343099 & -0.021139 & -1.428392 \\ \mathrm{H} & 0.247370 & -3.878134 & 2.128263 \\ \mathrm{H} & 0.963323 & 3.350224 & 2.717951\end{array}$

10

$\begin{array}{lrrr}\mathrm{O} & 0.038935 & 1.765416 & 1.417768 \\ \mathrm{O} & 1.463889 & -0.840928 & 1.480064 \\ \mathrm{O} & -1.494520 & -0.779224 & 1.483771 \\ \mathrm{O} & 1.474417 & 0.768855 & -0.978903 \\ \mathrm{O} & -1.441841 & 0.826950 & -0.978121 \\ \mathrm{O} & 2.991438 & -1.872149 & -0.963702 \\ \mathrm{O} & -0.037112 & -1.707587 & -0.920300 \\ \mathrm{O} & -3.069983 & -1.745382 & -0.958148 \\ \mathrm{~V} & 0.003005 & 0.052870 & 1.831010 \\ \mathrm{O} & 0.069421 & 3.464116 & -1.086181 \\ \mathrm{TI} & 0.036469 & 1.780570 & -0.439310 \\ \mathrm{TI} & -1.585122 & -0.889491 & -0.388083 \\ \mathrm{TI} & 1.544742 & -0.954538 & -0.391829 \\ \mathrm{H} & -3.961060 & -1.533819 & -1.251503 \\ \mathrm{H} & 3.902525 & -1.703361 & -1.221064 \\ \mathrm{H} & 0.118281 & 4.376741 & -0.787760\end{array}$

For Figure 3: 2-15

2

$\begin{array}{llll}0 & -0.490860 & 1.647806 & -0.389881\end{array}$ 


$\begin{array}{lrrr}\mathrm{O} & 1.902330 & 0.495591 & -1.475042 \\ \mathrm{O} & 1.710583 & 1.050747 & 1.322338 \\ \mathrm{O} & -0.529083 & -1.022930 & -1.310871 \\ \mathrm{O} & -0.695530 & -0.483744 & 1.456310 \\ \mathrm{O} & 1.951386 & -2.339533 & -2.609858 \\ \mathrm{O} & 1.662544 & -1.666986 & 0.386332 \\ \mathrm{O} & 1.588068 & -1.185677 & 3.418719 \\ \mathrm{~V} & -1.271779 & 0.079559 & -0.143312 \\ \mathrm{O} & 2.022154 & 3.428740 & -0.617730 \\ \mathrm{O} & -2.850134 & 0.144635 & -0.257017 \\ \mathrm{TI} & 1.315581 & -1.228421 & -1.343909 \\ \mathrm{TI} & 1.096927 & -0.618914 & 1.782683 \\ \mathrm{TI} & 1.359034 & 1.785024 & -0.300248 \\ \mathrm{H} & 1.196275 & -1.568643 & 4.209778 \\ \mathrm{H} & 2.158765 & -3.271049 & -2.731547 \\ \mathrm{H} & 2.413160 & 4.166953 & -0.140634 \\ \mathrm{C} & -6.496770 & 0.503478 & -0.108075 \\ \mathrm{H} & -7.545966 & 0.218528 & 0.040228 \\ \mathrm{H} & -6.387868 & 0.911698 & -1.127476 \\ \mathrm{H} & -6.245443 & 1.295424 & 0.618318 \\ \mathrm{O} & -5.715012 & -0.667790 & 0.085548 \\ \mathrm{H} & -4.782873 & -0.439712 & -0.042399 \\ & & & \end{array}$

3

$\begin{array}{lrrr}\mathrm{O} & -0.447240 & -1.242442 & 1.319434 \\ \mathrm{O} & -0.215264 & -1.141926 & -1.431752 \\ \mathrm{O} & -1.337894 & 0.915297 & -0.189833 \\ \mathrm{O} & 2.160989 & -0.884945 & 0.140911 \\ \mathrm{O} & 0.904104 & 1.279758 & 1.517234 \\ \mathrm{O} & 2.490197 & -0.909986 & -2.933255 \\ \mathrm{O} & 1.175709 & 1.340387 & -1.374785 \\ \mathrm{O} & -0.229125 & 3.743921 & -0.020003 \\ \mathrm{~V} & -1.377256 & -0.984780 & -0.126454 \\ \mathrm{O} & -2.308198 & -2.381829 & -0.378182 \\ \mathrm{O} & 2.009433 & -1.001102 & 3.202083 \\ \mathrm{C} & -4.171351 & 0.537463 & -0.150974 \\ \mathrm{H} & -5.183130 & 0.367072 & 0.245386 \\ \mathrm{H} & -4.230812 & 0.634077 & -1.246602 \\ \mathrm{H} & -3.774072 & 1.470467 & 0.267919 \\ \mathrm{O} & -3.352133 & -0.553018 & 0.233354 \\ \mathrm{H} & -3.262208 & -1.733932 & -0.173132 \\ \mathrm{TI} & 1.506812 & -0.432126 & -1.494977 \\ \mathrm{TI} & 1.227848 & -0.470415 & 1.664508 \\ \mathrm{TI} & 0.078878 & 1.956492 & -0.020990 \\ \mathrm{H} & -0.999069 & 4.310398 & -0.128294 \\ \mathrm{H} & 2.259158 & -0.586939 & 4.034245 \\ \mathrm{H} & 3.192656 & -1.535831 & -3.134770\end{array}$




\section{4}

$\begin{array}{lrrr}0 & 1.281442 & 0.696626 & -0.932264 \\ \mathrm{O} & -0.137841 & -1.437412 & -1.013550 \\ \mathrm{O} & 0.715950 & -0.938100 & 1.450012 \\ \mathrm{O} & -1.493416 & 0.941408 & -1.490527 \\ \mathrm{O} & -0.538212 & 1.656151 & 1.111791 \\ \mathrm{O} & -3.006779 & -1.769538 & -1.948674 \\ \mathrm{O} & -2.093399 & -0.709995 & 0.853908 \\ \mathrm{O} & -1.247646 & 0.084110 & 3.649042 \\ \mathrm{~V} & 1.476522 & -0.879618 & -0.100105 \\ \mathrm{O} & 2.124143 & -2.413852 & -0.798520 \\ \mathrm{O} & 0.118174 & 3.520666 & -1.266066 \\ \mathrm{C} & 4.141452 & 0.360583 & -0.163417 \\ \mathrm{H} & 4.694210 & -0.245791 & -0.899472 \\ \mathrm{H} & 4.831480 & 0.682099 & 0.629139 \\ \mathrm{H} & 3.714826 & 1.240436 & -0.672127 \\ \mathrm{O} & 3.101506 & -0.390594 & 0.414974 \\ \mathrm{H} & 3.048481 & -2.575036 & -0.562897 \\ \mathrm{TI} & -1.776718 & -0.841897 & -0.982028 \\ \mathrm{TI} & -0.840901 & 0.053780 & 1.887306 \\ \mathrm{TI} & -0.161946 & 1.823141 & -0.694854 \\ \mathrm{H} & -0.293703 & 4.087739 & -1.925658 \\ \mathrm{H} & -2.953859 & -2.556976 & -2.499604 \\ \mathrm{H} & -1.291096 & 0.748961 & 4.343188\end{array}$

5

$\begin{array}{lrrr}O & 0.285091 & 1.531507 & -0.669759 \\ \mathrm{O} & -0.125283 & -0.883515 & -1.437916 \\ \mathrm{O} & 0.834053 & -0.870187 & 1.025097 \\ \mathrm{O} & -2.398696 & 0.668796 & -1.074040 \\ \mathrm{O} & -1.408732 & 0.890037 & 1.601435 \\ \mathrm{O} & -2.681190 & -2.153899 & -2.441494 \\ \mathrm{O} & -1.882502 & -1.714433 & 0.566886 \\ \mathrm{O} & -1.003846 & -1.513914 & 3.460246 \\ \mathrm{~V} & 1.249674 & 0.048391 & -0.384235 \\ \mathrm{O} & 2.379705 & -0.656501 & -1.554538 \\ \mathrm{O} & -1.988261 & 3.483643 & 0.011035 \\ \mathrm{C} & 3.109961 & 2.256363 & 0.018571 \\ \mathrm{H} & 3.774151 & 2.130220 & -0.851322 \\ \mathrm{H} & 3.694381 & 2.635552 & 0.868602 \\ \mathrm{H} & 2.313990 & 2.974188 & -0.237605 \\ \mathrm{O} & 2.528991 & 1.023341 & 0.376752 \\ \mathrm{H} & 3.336312 & -0.563356 & -1.351797 \\ \mathrm{TI} & -1.827987 & -1.118354 & -1.208173 \\ \mathrm{TI} & -0.905045 & -0.833691 & 1.783228 \\ \mathrm{TI} & -1.436957 & 1.755803 & -0.040738\end{array}$




$\begin{array}{rrrr}\mathrm{H} & -2.697244 & 3.970322 & -0.421175 \\ \mathrm{H} & -2.368463 & -2.607010 & -3.230969 \\ \mathrm{H} & -1.267390 & -1.175869 & 4.321631 \\ \mathrm{C} & 5.089016 & -1.227063 & 0.422698 \\ \mathrm{O} & 5.061223 & -0.731213 & -0.683930 \\ \mathrm{H} & 6.053240 & -1.445510 & 0.926393 \\ \mathrm{H} & 4.159977 & -1.479313 & 0.972844\end{array}$

6

$\begin{array}{lrrr}\mathrm{O} & 0.534582 & 1.409220 & -0.759363 \\ \mathrm{O} & -0.379010 & -0.870579 & -1.548487 \\ \mathrm{O} & 0.792278 & -1.226055 & 0.762016 \\ \mathrm{O} & -2.268790 & 1.053584 & -0.841442 \\ \mathrm{O} & -0.944464 & 0.913778 & 1.692421 \\ \mathrm{O} & -3.270366 & -1.556039 & -2.244272 \\ \mathrm{O} & -2.036120 & -1.479106 & 0.612468 \\ \mathrm{O} & -0.803719 & -1.643710 & 3.393139 \\ \mathrm{~V} & 1.172666 & -0.256215 & -0.645591 \\ \mathrm{O} & 2.250754 & -0.878848 & -1.787269 \\ \mathrm{O} & -1.226589 & 3.676820 & 0.341456 \\ \mathrm{C} & 3.273100 & 1.737570 & 0.082218 \\ \mathrm{H} & 3.896842 & 1.650848 & -0.821420 \\ \mathrm{H} & 3.901882 & 2.049077 & 0.929492 \\ \mathrm{H} & 2.498581 & 2.494983 & -0.093204 \\ \mathrm{O} & 2.657213 & 0.495416 & 0.401531 \\ \mathrm{H} & 3.302553 & -1.032005 & -1.505075 \\ \mathrm{TI} & -2.084310 & -0.786792 & -1.106427 \\ \mathrm{TI} & -0.768320 & -0.885459 & 1.751784 \\ \mathrm{TI} & -1.016641 & 1.892228 & 0.123138 \\ \mathrm{H} & -1.829117 & 4.346081 & 0.002071 \\ \mathrm{H} & -3.201340 & -2.033189 & -3.077094 \\ \mathrm{H} & -0.920553 & -1.350836 & 4.302015 \\ \mathrm{C} & 4.098898 & -0.872088 & 0.375716 \\ \mathrm{O} & 4.461959 & -0.934341 & -0.826937 \\ \mathrm{H} & 4.739810 & -0.345285 & 1.098119 \\ \mathrm{H} & 3.390187 & -1.600227 & 0.797080\end{array}$

7

$\begin{array}{rrrr}0 & 0.609421 & 0.852772 & -1.153181 \\ 0 & -1.025395 & -1.418331 & -1.403755 \\ 0 & 0.596871 & -1.157066 & 0.872683 \\ 0 & -2.208821 & 1.133038 & -0.910429 \\ 0 & -0.565062 & 1.404721 & 1.416056 \\ 0 & -4.114076 & -1.258634 & -1.267332 \\ 0 & -2.236428 & -0.896230 & 1.122681 \\ 0 & -0.585722 & -0.678682 & 3.688691 \\ V & 0.604491 & -0.897622 & -0.875048\end{array}$




$\begin{array}{lrrr}\mathrm{O} & 1.765653 & -1.674958 & -1.625509 \\ \mathrm{O} & -0.501057 & 3.680438 & -0.643957 \\ \mathrm{C} & 4.208139 & 1.503357 & -0.030334 \\ \mathrm{H} & 4.413839 & 1.422341 & -1.106823 \\ \mathrm{H} & 5.154129 & 1.698282 & 0.503697 \\ \mathrm{H} & 3.515096 & 2.332701 & 0.149172 \\ \mathrm{O} & 3.581482 & 0.325451 & 0.471470 \\ \mathrm{H} & 3.788572 & -1.492810 & -1.364942 \\ \mathrm{TI} & -2.521458 & -0.668900 & -0.665638 \\ \mathrm{TI} & -0.689568 & -0.332537 & 1.922673 \\ \mathrm{TI} & -0.675207 & 1.910535 & -0.347174 \\ \mathrm{H} & -0.944702 & 4.377331 & -1.137027 \\ \mathrm{H} & -4.471825 & -1.920653 & -1.866661 \\ \mathrm{H} & -0.210261 & -0.276174 & 4.477916 \\ \mathrm{C} & 4.383284 & -0.834936 & 0.357449 \\ \mathrm{O} & 4.632182 & -1.216574 & -0.971336 \\ \mathrm{H} & 5.373141 & -0.663929 & 0.810868 \\ \mathrm{H} & 3.834910 & -1.610038 & 0.911595\end{array}$

8

$\begin{array}{lrrr}\mathrm{O} & 0.507587 & -1.918327 & -0.152235 \\ \mathrm{O} & 2.558171 & -0.152811 & 0.851524 \\ \mathrm{O} & 1.641851 & 0.105964 & -1.837383 \\ \mathrm{O} & -0.184185 & -0.004950 & 1.712991 \\ \mathrm{O} & -0.989879 & 0.289797 & -0.815891 \\ \mathrm{O} & 1.893791 & 1.877956 & 3.052660 \\ \mathrm{O} & 0.932438 & 2.099640 & 0.162395 \\ \mathrm{O} & -0.144465 & 2.492605 & -2.729318 \\ \mathrm{~V} & -0.855463 & -1.076315 & 0.508767 \\ \mathrm{O} & 3.429849 & -2.272667 & -1.170462 \\ \mathrm{O} & -1.674881 & -2.203758 & 1.469003 \\ \mathrm{TI} & 1.355306 & 1.041378 & 1.545895 \\ \mathrm{TI} & 0.299415 & 1.328248 & -1.411275 \\ \mathrm{TI} & 2.145650 & -1.123196 & -0.635603 \\ \mathrm{H} & -0.942730 & 2.700540 & -3.224318 \\ \mathrm{H} & 1.937056 & 2.783989 & 3.373974 \\ \mathrm{H} & 3.889738 & -2.475586 & -1.991165 \\ \mathrm{C} & -3.705877 & -0.644831 & -0.693304 \\ \mathrm{H} & -3.358193 & 0.038431 & -1.487643 \\ \mathrm{H} & -4.596456 & -1.202053 & -1.042192 \\ \mathrm{C} & -5.013299 & 1.090603 & 0.236132 \\ \mathrm{H} & -5.962135 & 0.645441 & -0.111157 \\ \mathrm{H} & -5.187152 & 1.603140 & 1.188372 \\ \mathrm{H} & -4.670505 & 1.825811 & -0.513254 \\ \mathrm{O} & -4.029677 & 0.097188 & 0.473178 \\ \mathrm{O} & -2.708622 & -1.552255 & -0.375312 \\ \mathrm{H} & -2.582918 & -2.127648 & 0.741181\end{array}$


9

$\begin{array}{lrrr}\mathrm{O} & 0.179348 & -1.532138 & 0.735020 \\ \mathrm{O} & -2.540329 & -1.137193 & -0.051896 \\ \mathrm{O} & -1.387180 & 0.660815 & 1.831533 \\ \mathrm{O} & -0.330021 & -0.541435 & -1.671133 \\ \mathrm{O} & 0.804727 & 1.152401 & -0.007543 \\ \mathrm{O} & -3.009683 & -0.279820 & -3.038276 \\ \mathrm{O} & -1.888504 & 1.620192 & -0.806786 \\ \mathrm{O} & -0.721381 & 3.584017 & 1.199476 \\ \mathrm{~V} & 1.156146 & -0.527504 & -0.287459 \\ \mathrm{O} & -2.092189 & -2.163401 & 2.773219 \\ \mathrm{O} & 2.056162 & -1.156648 & -1.727195 \\ \mathrm{TI} & -2.002235 & -0.108004 & -1.531953 \\ \mathrm{TI} & -0.817577 & 1.882875 & 0.593839 \\ \mathrm{TI} & -1.530510 & -1.111267 & 1.413432 \\ \mathrm{H} & -1.040541 & 4.438207 & 0.891586 \\ \mathrm{H} & -2.821968 & -0.564095 & -3.938011 \\ \mathrm{H} & -2.799796 & -2.805227 & 2.890548 \\ \mathrm{C} & 4.040134 & -0.775272 & 0.247043 \\ \mathrm{H} & 4.537869 & -1.318434 & 1.072467 \\ \mathrm{H} & 4.134492 & -1.340669 & -0.699150 \\ \mathrm{C} & 5.955553 & 0.490591 & -0.231513 \\ \mathrm{H} & 6.118839 & -0.014291 & -1.199378 \\ \mathrm{H} & 6.281817 & 1.532905 & -0.308808 \\ \mathrm{H} & 6.554463 & -0.018827 & 0.543787 \\ \mathrm{O} & 4.577449 & 0.518939 & 0.111969 \\ \mathrm{O} & 2.692473 & -0.658279 & 0.562755 \\ \mathrm{H} & 1.449472 & -1.207788 & -2.480824\end{array}$

10

$\begin{array}{rrrr}\mathrm{O} & 1.086992 & -0.053866 & -1.111766 \\ \mathrm{O} & -1.535554 & -1.269276 & -1.483020 \\ \mathrm{O} & -0.145180 & -1.715380 & 0.960383 \\ \mathrm{O} & -1.176783 & 1.443815 & -0.929229 \\ \mathrm{O} & 0.061073 & 1.122871 & 1.487959 \\ \mathrm{O} & -3.971795 & 0.742163 & -1.645734 \\ \mathrm{O} & -2.498200 & -0.116189 & 1.025076 \\ \mathrm{O} & -1.128627 & -0.699825 & 3.681849 \\ \mathrm{~V} & 0.640212 & 1.505694 & -0.116362 \\ \mathrm{O} & 0.887973 & -3.114043 & -1.542522 \\ \mathrm{O} & 0.670160 & 3.191079 & -0.721787 \\ \mathrm{C} & 3.363831 & 0.668716 & -0.394052 \\ \mathrm{H} & 2.273721 & -0.080222 & -1.034153 \\ \mathrm{H} & 3.971845 & 1.101640 & -1.207876 \\ \mathrm{C} & 5.215096 & -0.743153 & -0.036931 \\ \mathrm{H} & 5.909929 & -0.018741 & -0.490446\end{array}$




$\begin{array}{lrrr}\mathrm{H} & 5.708013 & -1.248849 & 0.798496 \\ \mathrm{H} & 4.914004 & -1.486852 & -0.790902 \\ \mathrm{O} & 4.075670 & -0.080081 & 0.512099 \\ \mathrm{O} & 2.448655 & 1.446758 & 0.141219 \\ \mathrm{H} & -0.182607 & 3.444436 & -1.107960 \\ \mathrm{TI} & -2.426329 & 0.252943 & -0.807009 \\ \mathrm{TI} & -0.961858 & -0.371789 & 1.910801 \\ \mathrm{TI} & 0.072259 & -1.654746 & -0.844634 \\ \mathrm{H} & -4.859875 & 0.372540 & -1.666438 \\ \mathrm{H} & 0.673774 & -3.727955 & -2.252506 \\ \mathrm{H} & -1.851922 & -0.705543 & 4.316312\end{array}$

11

$0 \quad 0.605324$

o $\quad-2.282796$

$0 \quad-1.310627$

$-0.763029$

$-1.409743$

$-0.114460$

$-1.490891$

$-1.919793$

0.480801

$-0.498229 \quad 1.804155$

$-0.490422$

0.051647

1.497761

276977

2.809855

$-0.761408$

$-2.334746$

0.612041

$-1.284134$

1.335826

$-1.344328$

0.715505

3.521222

1.098138

$-2.906809$

$-0.070063$

$-1.452089$

2.162996

2.123016

$-2.375244$

$-0.557772$

4.092396

0.163216

$-0.041171$

$-1.399501$

1.217089

$-1.530492$

4.230729

0.061556

$-0.330995$

6.462759

0.134159

$-0.042408$

$-0.575121$

1.060443

0.942091

7.058563

6.348758

5.178618

$-0.578761$

2.981202

$-0.318872$

$-0.703819$

$-0.482714$

0.121960

0.120865

2.889449

$-0.872393$

1.408635

$-0.344196$

$-0.647620$

1.825272

$-1.486804$

$-1.267651$

2.946831

$-0.601746$

$-3.060294$

$-3.130759$

$-1.169788$

4.184718

12

$\begin{array}{rrrr}0 & 0.386133 & -1.902026 & -0.821908 \\ 0 & 1.478872 & -0.177407 & 1.180294 \\ 0 & 1.116200 & 0.896554 & -1.474324 \\ 0 & -1.229643 & -0.885873 & 1.444896 \\ 0 & -1.651746 & 0.137918 & -1.167535\end{array}$




$\begin{array}{lrrr}\mathrm{O} & 0.164489 & 0.826603 & 3.696242 \\ \mathrm{O} & -0.451062 & 1.849823 & 0.771228 \\ \mathrm{O} & -1.004139 & 3.057276 & -1.972379 \\ \mathrm{~V} & 1.886092 & -0.496228 & -0.678682 \\ \mathrm{O} & 3.479975 & -1.289874 & -0.851635 \\ \mathrm{O} & -2.630127 & -2.684812 & -0.553864 \\ \mathrm{H} & 4.299924 & -1.046030 & -1.293119 \\ \mathrm{H} & 0.449244 & -2.475685 & -1.596502 \\ \mathrm{TI} & 0.040574 & 0.437864 & 1.918413 \\ \mathrm{TI} & -1.405040 & -1.386169 & -0.244819 \\ \mathrm{TI} & -0.521675 & 1.604780 & -0.994645 \\ \mathrm{H} & 0.867419 & 0.699199 & 4.340835 \\ \mathrm{H} & -1.321641 & 3.935301 & -1.738873 \\ \mathrm{H} & -3.451596 & -2.767480 & -1.047640\end{array}$

\section{3}

$\begin{array}{lrrr}0 & 2.134471 & -0.034049 & -0.348619 \\ \mathrm{O} & -0.351250 & 1.709645 & -1.292180 \\ \mathrm{O} & -0.323058 & -1.181894 & -1.741163 \\ \mathrm{O} & 0.247007 & 1.214678 & 1.573124 \\ \mathrm{O} & 0.196111 & -1.675514 & 1.118626 \\ \mathrm{O} & -2.114052 & 2.969942 & 0.834747 \\ \mathrm{O} & -2.051683 & -0.046840 & 0.332227 \\ \mathrm{O} & -2.194456 & -3.043590 & -0.175725 \\ \mathrm{~V} & 0.624531 & 0.273449 & -1.607877 \\ \mathrm{O} & 2.390577 & 0.545878 & -2.477480 \\ \mathrm{O} & 2.524154 & -0.464401 & 2.621917 \\ \mathrm{H} & 2.689131 & 0.182150 & -3.316968 \\ \mathrm{H} & 2.728507 & 0.164640 & -1.328226 \\ \mathrm{TI} & 1.321602 & -0.246541 & 1.291216 \\ \mathrm{TI} & -1.100110 & 1.540501 & 0.391051 \\ \mathrm{TI} & -1.160399 & -1.560135 & -0.101688 \\ \mathrm{H} & -3.129947 & -3.253654 & -0.251799 \\ \mathrm{H} & 2.894803 & -1.185866 & 3.138764 \\ \mathrm{H} & -2.553325 & 3.708410 & 0.402862\end{array}$

\section{4}

$\begin{array}{rrrr}0 & -0.922819 & 0.024003 & -1.933579 \\ 0 & 1.122765 & -1.651487 & -0.730316 \\ 0 & 1.321494 & 1.483843 & -0.764547 \\ 0 & -1.477974 & -1.358720 & 0.590435 \\ 0 & -1.307817 & 1.528360 & 0.567618 \\ 0 & 0.676279 & -3.041096 & 1.927905 \\ 0 & 0.839680 & -0.021448 & 1.765740 \\ 0 & 1.053815 & 3.005757 & 1.824936 \\ \mathrm{~V} & 0.773679 & -0.073920 & -1.489675 \\ 0 & 2.907797 & -0.168288 & -2.160739\end{array}$




$\begin{array}{lrrr}\mathrm{O} & -3.725182 & 0.237773 & -0.738915 \\ \mathrm{H} & 3.345800 & 0.588996 & -1.742006 \\ \mathrm{H} & 3.299705 & -0.968076 & -1.778425 \\ \mathrm{TI} & -1.951699 & 0.121376 & -0.388007 \\ \mathrm{TI} & 0.300321 & -1.568415 & 0.935801 \\ \mathrm{TI} & 0.505377 & 1.552436 & 0.887516 \\ \mathrm{H} & -4.343099 & -0.021139 & -1.428392 \\ \mathrm{H} & 0.247370 & -3.878134 & 2.128263 \\ \mathrm{H} & 0.963323 & 3.350224 & 2.717951\end{array}$

\section{5}

$\begin{array}{lrrr}\mathrm{O} & 0.038935 & 1.765416 & 1.417768 \\ \mathrm{O} & 1.463889 & -0.840928 & 1.480064 \\ \mathrm{O} & -1.494520 & -0.779224 & 1.483771 \\ \mathrm{O} & 1.474417 & 0.768855 & -0.978903 \\ \mathrm{O} & -1.441841 & 0.826950 & -0.978121 \\ \mathrm{O} & 2.991438 & -1.872149 & -0.963702 \\ \mathrm{O} & -0.037112 & -1.707587 & -0.920300 \\ \mathrm{O} & -3.069983 & -1.745382 & -0.958148 \\ \mathrm{~V} & 0.003005 & 0.052870 & 1.831010 \\ \mathrm{O} & 0.069421 & 3.464116 & -1.086181 \\ \mathrm{TI} & 0.036469 & 1.780570 & -0.439310 \\ \mathrm{TI} & -1.585122 & -0.889491 & -0.388083 \\ \mathrm{TI} & 1.544742 & -0.954538 & -0.391829 \\ \mathrm{H} & -3.961060 & -1.533819 & -1.251503 \\ \mathrm{H} & 3.902525 & -1.703361 & -1.221064 \\ \mathrm{H} & 0.118281 & 4.376741 & -0.787760\end{array}$

\section{For Figure 4: 2-11}

\section{$2 a$}

$\begin{array}{lr}O & -0.490860 \\ O & 1.902330 \\ O & 1.710583 \\ O & -0.529083 \\ O & -0.695530 \\ O & 1.951386 \\ \mathrm{O} & 1.662544 \\ \mathrm{O} & 1.588068 \\ \mathrm{~V} & -1.271779 \\ \mathrm{O} & 2.022154 \\ \mathrm{O} & -2.850134 \\ \mathrm{TI} & 1.315581 \\ \mathrm{TI} & 1.096927 \\ \mathrm{TI} & 1.359034 \\ \mathrm{H} & 1.196275 \\ \mathrm{H} & 2.158765\end{array}$

$$
\begin{array}{rr}
1.647806 & -0.389881 \\
0.495591 & -1.475042 \\
1.050747 & 1.322338 \\
-1.022930 & -1.310871 \\
-0.483744 & 1.456310 \\
-2.339533 & -2.609858 \\
-1.666986 & 0.386332 \\
-1.185677 & 3.418719 \\
0.079559 & -0.143312 \\
3.428740 & -0.617730 \\
0.144635 & -0.257017 \\
-1.228421 & -1.343909 \\
-0.618914 & 1.782683 \\
1.785024 & -0.300248 \\
-1.568643 & 4.209778 \\
-3.271049 & -2.731547
\end{array}
$$




$\begin{array}{rrrr}\mathrm{H} & 2.413160 & 4.166953 & -0.140634 \\ \mathrm{C} & -6.496770 & 0.503478 & -0.108075 \\ \mathrm{H} & -7.545966 & 0.218528 & 0.040228 \\ \mathrm{H} & -6.387868 & 0.911698 & -1.127476 \\ \mathrm{H} & -6.245443 & 1.295424 & 0.618318 \\ \mathrm{O} & -5.715012 & -0.667790 & 0.085548 \\ \mathrm{H} & -4.782873 & -0.439712 & -0.042399\end{array}$

\section{2b}

0

0

0

O

0

0

O

1.025712

$$
-0.576899
$$

1.023020

$-1.800157$

$-0.118163$

$-3.650254$

$-1.778389$

$-0.071999$

1.053797

2.161557

$-0.105916$

4.644019

5.680350

4.524161

3.684717

$-2.083283$

$-0.228082$

$-0.231262$

$-0.433961$

0.076829

$-4.356883$

3a

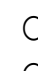

0

0

0

0

0

0

O

V

O

0

C

$\mathrm{H}$

$\mathrm{H}$

$\mathrm{H}$

$$
\begin{array}{r}
-1.449828 \\
0.020931 \\
1.454109 \\
-1.425682 \\
-0.042228 \\
0.058615 \\
1.444359 \\
3.031556 \\
0.013498 \\
0.026768 \\
-3.120009 \\
0.007146 \\
0.002159 \\
0.026238 \\
-0.007865 \\
0.010019 \\
-1.610648 \\
1.585952 \\
3.920917 \\
-3.354328 \\
-0.551243
\end{array}
$$

0.237757

1.977071

0.204068

$-0.144771$

$-1.991351$

1.814990

$-0.197715$

$-2.201410$

1.257287

2.386017

$-2.080890$

0.517513

0.114860

1.620352

$-0.220084$

0.917251

$-1.096060$

$-1.131138$

$-2.260173$

$-2.995994$

2.047640

1. 319434

$-1.431752$

$-0.189833$

0.140911

1.517234

$-2.933255$

$-1.374785$

$-0.020003$

$-0.126454$

$-0.378182$

3.202083

$-0.150974$

0.245386

$-1.246602$

0.267919 


$\begin{array}{lrrr}\mathrm{O} & -3.352133 & -0.553018 & 0.233354 \\ \mathrm{H} & -3.262208 & -1.733932 & -0.173132 \\ \mathrm{TI} & 1.506812 & -0.432126 & -1.494977 \\ \mathrm{TI} & 1.227848 & -0.470415 & 1.664508 \\ \mathrm{TI} & 0.078878 & 1.956492 & -0.020990 \\ \mathrm{H} & -0.999069 & 4.310398 & -0.128294 \\ \mathrm{H} & 2.259158 & -0.586939 & 4.034245 \\ \mathrm{H} & 3.192656 & -1.535831 & -3.134770\end{array}$

3b

$\begin{array}{lrrr}\mathrm{O} & 0.750647 & 0.025823 & -1.849986 \\ \mathrm{O} & -0.875477 & 1.564056 & -0.415229 \\ \mathrm{O} & 1.642964 & -1.529956 & 0.335732 \\ \mathrm{O} & -0.212893 & -0.028158 & 1.902391 \\ \mathrm{O} & 3.791735 & -0.146111 & -1.404689 \\ \mathrm{O} & 1.815782 & 1.350577 & 0.425119 \\ \mathrm{O} & -0.089160 & 3.049575 & 2.146552 \\ \mathrm{~V} & -0.992062 & 0.083083 & -1.439858 \\ \mathrm{O} & -2.062849 & 0.144576 & -2.746228 \\ \mathrm{O} & -0.397586 & -3.099056 & 2.003546 \\ \mathrm{C} & -3.313267 & 0.454058 & -0.393945 \\ \mathrm{H} & -3.451736 & 1.553929 & -0.182536 \\ \mathrm{H} & -2.832149 & 0.219612 & -1.939264 \\ \mathrm{O} & -3.867826 & -0.384624 & 0.252864 \\ \mathrm{TI} & 2.147713 & -0.099999 & -0.658610 \\ \mathrm{TI} & -0.051874 & -1.611982 & 1.045267 \\ \mathrm{TI} & 0.154868 & 1.598644 & 1.100217 \\ \mathrm{H} & 0.390189 & 3.848011 & 2.387655 \\ \mathrm{H} & -0.885342 & -3.335495 & 2.798775 \\ \mathrm{H} & 4.491642 & -0.787580 & -1.559919\end{array}$

$4 a$

$\begin{array}{rrrr}\mathrm{O} & 1.281442 & 0.696626 & -0.932264 \\ \mathrm{O} & -0.137841 & -1.437412 & -1.013550 \\ \mathrm{O} & 0.715950 & -0.938100 & 1.450012 \\ \mathrm{O} & -1.493416 & 0.941408 & -1.490527 \\ \mathrm{O} & -0.538212 & 1.656151 & 1.111791 \\ \mathrm{O} & -3.006779 & -1.769538 & -1.948674 \\ \mathrm{O} & -2.093399 & -0.709995 & 0.853908 \\ \mathrm{O} & -1.247646 & 0.084110 & 3.649042 \\ \mathrm{~V} & 1.476522 & -0.879618 & -0.100105 \\ \mathrm{O} & 2.124143 & -2.413852 & -0.798520 \\ \mathrm{O} & 0.118174 & 3.520666 & -1.266066 \\ \mathrm{C} & 4.141452 & 0.360583 & -0.163417 \\ \mathrm{H} & 4.694210 & -0.245791 & -0.899472 \\ \mathrm{H} & 4.831480 & 0.682099 & 0.629139 \\ \mathrm{H} & 3.714826 & 1.240436 & -0.672127\end{array}$




$\begin{array}{lrrr}\mathrm{O} & 3.101506 & -0.390594 & 0.414974 \\ \mathrm{H} & 3.048481 & -2.575036 & -0.562897 \\ \mathrm{TI} & -1.776718 & -0.841897 & -0.982028 \\ \mathrm{TI} & -0.840901 & 0.053780 & 1.887306 \\ \mathrm{TI} & -0.161946 & 1.823141 & -0.694854 \\ \mathrm{H} & -0.293703 & 4.087739 & -1.925658 \\ \mathrm{H} & -2.953859 & -2.556976 & -2.499604 \\ \mathrm{H} & -1.291096 & 0.748961 & 4.343188\end{array}$

$4 b$

$\begin{array}{lrrr}\mathrm{O} & 1.142503 & 1.150578 & -0.402671 \\ \mathrm{O} & 0.454528 & -1.251192 & -1.371916 \\ \mathrm{O} & 0.823810 & -0.703726 & 1.492457 \\ \mathrm{O} & -1.442889 & 0.864635 & -1.492507 \\ \mathrm{O} & -1.066936 & 1.400699 & 1.339944 \\ \mathrm{O} & -2.362183 & -1.878187 & -2.498628 \\ \mathrm{O} & -1.834934 & -1.192593 & 0.434687 \\ \mathrm{O} & -1.548015 & -0.769657 & 3.460799 \\ \mathrm{~V} & 1.544751 & -0.697328 & -0.121598 \\ \mathrm{O} & -0.507418 & 3.703964 & -0.655365 \\ \mathrm{C} & 3.311743 & 0.295424 & -0.595458 \\ \mathrm{O} & 4.133820 & 0.597112 & 0.218818 \\ \mathrm{H} & 3.403102 & 0.533372 & -1.686794 \\ \mathrm{O} & 2.697413 & -2.056294 & -0.025033 \\ \mathrm{H} & 3.624288 & -2.061088 & 0.244406 \\ \mathrm{TI} & -0.963178 & -0.339501 & 1.805507 \\ \mathrm{TI} & -1.388567 & -0.923975 & -1.313176 \\ \mathrm{TI} & -0.455008 & 1.921361 & -0.326508 \\ \mathrm{H} & 0.145295 & 4.391262 & -0.820430 \\ \mathrm{H} & -2.906669 & -1.693963 & -3.270303 \\ \mathrm{H} & -1.834770 & -0.299794 & 4.249834\end{array}$

\section{$5 a$}

0

O

O

O

0

O

O

O

V

O

O

C

$\mathrm{H}$

$\mathrm{H}$
0.471112

0.167145

0.403793

$-2.043899$

$-1.829259$

$-2.051922$

$-2.102704$

$-1.970338$

1.257895

2. 622526

$-1.917304$

2.939991

3. 867440

2.253833
$-0.180940$

$-1.401209$

1.151675

$-1.421411$

1.409843

$-3.241054$

$-0.089712$

2. 945738

0.084245

$-0.949314$

0.168112

2. 479634

2.140754

2. 887568
$-1.592184$

0.721132

1.062800

$-0.985785$

$-0.772409$

1.601793

1. 641431

1.889102

$-0.069168$

0.418411

$-3.568961$

0.084902

0.572150

0.844896 


$\begin{array}{lrrr}\mathrm{H} & 3.183505 & 3.253411 & -0.656306 \\ \mathrm{O} & 2.316562 & 1.401478 & -0.573461 \\ \mathrm{H} & 3.518233 & -0.591382 & 0.246127 \\ \mathrm{TI} & -1.530309 & -1.678065 & 0.815121 \\ \mathrm{TI} & -1.434815 & 1.443620 & 1.020866 \\ \mathrm{TI} & -1.395899 & -0.005024 & -1.841087 \\ \mathrm{H} & -2.435794 & -0.381530 & -4.165168 \\ \mathrm{H} & -1.541184 & -3.956463 & 1.993713 \\ \mathrm{H} & -2.547035 & 3.111768 & 2.641309 \\ \mathrm{C} & 5.892995 & -1.502412 & 0.047924 \\ \mathrm{O} & 5.361631 & -0.412804 & 0.078445 \\ \mathrm{H} & 6.995204 & -1.601099 & -0.029641 \\ \mathrm{H} & 5.300059 & -2.438893 & 0.096499\end{array}$

5b

$\begin{array}{lrrr}\mathrm{O} & 1.398500 & 1.378818 & -0.322691 \\ \mathrm{O} & -0.501386 & 0.720031 & 1.440277 \\ \mathrm{O} & -0.764244 & 0.313717 & -1.463057 \\ \mathrm{O} & 1.878279 & -0.793918 & 1.430390 \\ \mathrm{O} & 1.574167 & -1.228507 & -1.419933 \\ \mathrm{O} & -0.309670 & -1.645043 & 3.416002 \\ \mathrm{O} & -0.475634 & -1.988610 & 0.371436 \\ \mathrm{O} & -0.862930 & -2.598845 & -2.611243 \\ \mathrm{~V} & -0.494520 & 1.440520 & -0.169562 \\ \mathrm{O} & -2.082738 & 2.305874 & -0.191069 \\ \mathrm{O} & 4.179624 & 0.120250 & -0.422364 \\ \mathrm{C} & 0.163356 & 3.378747 & -0.553074 \\ \mathrm{H} & 0.477144 & 3.544317 & -1.615891 \\ \mathrm{H} & -3.389727 & 0.645591 & -0.425308 \\ \mathrm{O} & 0.221081 & 4.224235 & 0.291472 \\ \mathrm{C} & -4.826208 & -0.475686 & 0.312436 \\ \mathrm{H} & -5.682630 & 0.088222 & -0.093402 \\ \mathrm{H} & -4.677479 & -0.188922 & 1.366870 \\ \mathrm{H} & -5.067279 & -1.544881 & 0.274292 \\ \mathrm{O} & -3.648365 & -0.286367 & -0.464073 \\ \mathrm{H} & -2.266387 & 3.207168 & 0.100814 \\ \mathrm{TI} & 2.393400 & -0.094778 & -0.201204 \\ \mathrm{TI} & 0.119164 & -0.992426 & 1.785284 \\ \mathrm{TI} & -0.208222 & -1.478380 & -1.357843 \\ \mathrm{H} & -0.513620 & -3.073571 & -3.371850 \\ \mathrm{H} & 4.773385 & 0.865959 & -0.552807 \\ \mathrm{H} & 0.148680 & -1.824433 & 4.242635\end{array}$

\section{$6 a$}

$\begin{array}{rrrr}0 & 0.118523 & 1.644471 & -0.726657 \\ 0 & 0.392029 & -0.949848 & -1.160095 \\ 0 & 0.832513 & -0.076474 & 1.291372\end{array}$




$\begin{array}{lrrr}\mathrm{O} & -2.205803 & 0.093396 & -1.336396 \\ \mathrm{O} & -1.780521 & 1.048019 & 1.333972 \\ \mathrm{O} & -1.739940 & -2.913961 & -2.074313 \\ \mathrm{O} & -1.447326 & -1.748772 & 0.797382 \\ \mathrm{O} & -1.019795 & -0.838361 & 3.658637 \\ \mathrm{~V} & 1.352356 & 0.472475 & -0.304119 \\ \mathrm{O} & 2.478061 & 0.108261 & -2.017057 \\ \mathrm{O} & -2.649879 & 3.062920 & -0.818485 \\ \mathrm{C} & 4.084228 & 1.656162 & 0.301296 \\ \mathrm{H} & 4.451567 & 2.196590 & -0.585575 \\ \mathrm{H} & 4.589871 & 0.684379 & 0.398860 \\ \mathrm{H} & 4.283062 & 2.267347 & 1.192757 \\ \mathrm{O} & 2.691123 & 1.464341 & 0.201388 \\ \mathrm{H} & 3.258225 & 0.676752 & -2.072373 \\ \mathrm{TI} & -1.265848 & -1.502997 & -1.031997 \\ \mathrm{TI} & -0.891651 & -0.433314 & 1.900029 \\ \mathrm{TI} & -1.731260 & 1.552959 & -0.431116 \\ \mathrm{H} & -3.338740 & 3.301753 & -1.446761 \\ \mathrm{H} & -1.288819 & -3.423872 & -2.754447 \\ \mathrm{H} & -1.314055 & -0.402359 & 4.463934 \\ \mathrm{C} & 2.871138 & -1.558268 & 0.015686 \\ \mathrm{O} & 3.975862 & -1.538318 & 0.511319 \\ \mathrm{H} & 2.703763 & -0.706881 & -1.151621 \\ \mathrm{H} & 2.212667 & -2.459676 & 0.147383 \\ & & & \end{array}$

6b

$\begin{array}{rrrr}\mathrm{O} & 0.333894 & 1.632984 & -0.024415 \\ \mathrm{O} & 0.908467 & -0.765659 & -1.034835 \\ \mathrm{O} & 0.207335 & -0.384266 & 1.707503 \\ \mathrm{O} & -1.525790 & 0.423348 & -1.809812 \\ \mathrm{O} & -2.216694 & 0.839796 & 0.950031 \\ \mathrm{O} & -0.909720 & -2.378524 & -2.934865 \\ \mathrm{O} & -1.597461 & -1.804704 & -0.011769 \\ \mathrm{O} & -2.346093 & -1.523665 & 2.957655 \\ \mathrm{~V} & 1.350737 & 0.188764 & 0.396417 \\ \mathrm{O} & 2.607632 & 0.479637 & 2.112822 \\ \mathrm{O} & -2.101274 & 3.307512 & -0.872849 \\ \mathrm{C} & 2.684481 & 1.747816 & -0.096151 \\ \mathrm{H} & 2.585679 & 2.679478 & 0.512432 \\ \mathrm{H} & 3.227742 & -0.390500 & 1.375430 \\ \mathrm{O} & 3.415825 & 1.635997 & -1.033921 \\ \mathrm{C} & 3.853132 & -1.550385 & -0.503412 \\ \mathrm{H} & 4.424825 & -2.363757 & -0.036435 \\ \mathrm{H} & 4.538265 & -0.780574 & -0.883358 \\ \mathrm{H} & 3.257802 & -1.948747 & -1.333193 \\ \mathrm{O} & 2.963656 & -1.001322 & 0.461269 \\ \mathrm{H} & 3.071230 & 1.309842 & 2.273511\end{array}$




$\begin{array}{lrrr}\text { TI } & -1.454983 & 1.665427 & -0.457526 \\ \text { TI } & -1.527038 & -0.785311 & 1.519032 \\ \text { TI } & -0.807219 & -1.222971 & -1.543237 \\ \text { H } & -1.078028 & -2.307944 & -3.879497 \\ \text { H } & -2.074176 & -1.747397 & 3.853262 \\ \text { H } & -2.800907 & 3.877198 & -0.538819\end{array}$

7

$\begin{array}{lrrr}\mathrm{O} & 0.219120 & -1.480032 & -1.088421 \\ \mathrm{O} & 0.367304 & -0.555777 & 1.430811 \\ \mathrm{O} & 0.860612 & 1.347766 & -0.353010 \\ \mathrm{O} & -2.174092 & -1.290427 & 0.440338 \\ \mathrm{O} & -1.686133 & 0.653113 & -1.580895 \\ \mathrm{O} & -1.921542 & -0.569833 & 3.450989 \\ \mathrm{O} & -1.569320 & 1.464725 & 1.142136 \\ \mathrm{O} & -1.085784 & 3.619151 & -0.985476 \\ \mathrm{~V} & 1.308568 & -0.395440 & -0.225963 \\ \mathrm{O} & 3.440354 & 2.153474 & 0.721093 \\ \mathrm{O} & -2.444473 & -2.176952 & -2.485336 \\ \mathrm{C} & 4.147514 & -0.449184 & -1.371817 \\ \mathrm{H} & 4.375260 & -0.387127 & -2.444537 \\ \mathrm{H} & 4.602591 & 0.400401 & -0.844364 \\ \mathrm{H} & 2.557386 & 2.226480 & 0.321717 \\ \mathrm{O} & 2.742396 & -0.402259 & -1.220034 \\ \mathrm{H} & 3.634776 & 3.025015 & 1.082954 \\ \mathrm{H} & 4.536560 & -1.397152 & -0.973959 \\ \mathrm{C} & 2.638226 & -1.116070 & 1.198719 \\ \mathrm{O} & 2.941727 & -2.267775 & 1.273768 \\ \mathrm{H} & 3.016734 & -0.307998 & 1.872865 \\ \mathrm{TI} & -0.901496 & 1.888435 & -0.481350 \\ \mathrm{TI} & -1.353102 & -0.269505 & 1.752740 \\ \mathrm{TI} & -1.599083 & -1.146240 & -1.260863 \\ \mathrm{H} & -3.091377 & -2.888783 & -2.462979 \\ \mathrm{H} & -1.512809 & -0.897115 & 4.258088 \\ \mathrm{H} & -1.671024 & 4.352344 & -0.773369\end{array}$

8

$\begin{array}{rrrr}0 & 0.890563 & -0.514966 & 1.379212 \\ 0 & 0.519632 & 1.552298 & -0.262251 \\ 0 & 0.547325 & -0.792602 & -1.553769 \\ 0 & -1.578713 & 0.867803 & 1.518615 \\ 0 & -1.515541 & -1.707546 & 0.290185 \\ 0 & -1.785229 & 3.487999 & -0.168836 \\ 0 & -1.923729 & 0.598120 & -1.330497 \\ 0 & -1.978085 & -2.142195 & -2.705625 \\ \mathrm{~V} & 1.495048 & -0.089427 & -0.245213 \\ 0 & -1.216112 & -1.586362 & 3.342594\end{array}$




$\begin{array}{lrrr}\mathrm{C} & 4.310889 & -1.249554 & 0.039725 \\ \mathrm{H} & 4.367777 & -1.950348 & 0.885429 \\ \mathrm{H} & 4.840712 & -1.676866 & -0.823541 \\ \mathrm{O} & 2.953640 & -1.062217 & -0.296910 \\ \mathrm{H} & 4.776268 & -0.297469 & 0.329487 \\ \mathrm{C} & 2.826844 & 1.456416 & -0.620611 \\ \mathrm{O} & 3.556658 & 1.906630 & 0.212705 \\ \mathrm{H} & 2.775268 & 1.839787 & -1.672556 \\ \mathrm{TI} & -1.238809 & 1.759222 & -0.055596 \\ \mathrm{TI} & -0.887252 & -0.792184 & 1.748918 \\ \mathrm{TI} & -1.284414 & -1.084365 & -1.411126 \\ \mathrm{H} & -2.586874 & -2.033973 & -3.442556 \\ \mathrm{H} & -1.525928 & -1.312981 & 4.211200 \\ \mathrm{H} & -2.592543 & 3.973018 & 0.026055\end{array}$

9

$\begin{array}{lrrr}\mathrm{O} & 1.113294 & -0.595241 & 1.171681 \\ \mathrm{O} & 0.414438 & 1.700494 & -0.237139 \\ \mathrm{O} & 0.399768 & -0.609262 & -1.740486 \\ \mathrm{O} & -1.399740 & 0.678120 & 1.707136 \\ \mathrm{O} & -1.358965 & -1.767387 & 0.246227 \\ \mathrm{O} & -2.117573 & 3.345470 & 0.367831 \\ \mathrm{O} & -2.088472 & 0.637502 & -1.094741 \\ \mathrm{O} & -2.193594 & -1.994817 & -2.687947 \\ \mathrm{~V} & 1.333250 & 0.162653 & -0.454049 \\ \mathrm{O} & -0.686120 & -1.918451 & 3.244003 \\ \mathrm{C} & 3.951656 & -1.239188 & 0.131321 \\ \mathrm{H} & 3.363896 & -1.991492 & 0.667979 \\ \mathrm{H} & 4.760895 & -1.715415 & -0.436048 \\ \mathrm{O} & 3.110245 & -0.552799 & -0.810755 \\ \mathrm{H} & 4.364605 & -0.513663 & 0.845334 \\ \mathrm{C} & 3.123457 & 1.149787 & -0.778850 \\ \mathrm{O} & 3.600666 & 1.673914 & 0.203479 \\ \mathrm{H} & 3.307574 & 1.386828 & -1.841260 \\ \mathrm{TI} & -1.347055 & 1.717924 & 0.201555 \\ \mathrm{TI} & -0.595654 & -0.962197 & 1.711032 \\ \mathrm{TI} & -1.386235 & -1.004790 & -1.408705 \\ \mathrm{H} & -2.890290 & -1.872830 & -3.340034 \\ \mathrm{H} & -1.312235 & -2.058633 & 3.960467 \\ \mathrm{H} & -1.824690 & 4.259648 & 0.432151\end{array}$

10

$\begin{array}{rrrr}0 & 0.744526 & -1.639352 & 0.161566 \\ 0 & -0.515219 & 0.140573 & 2.068786 \\ 0 & 0.975551 & 1.478005 & -0.034228 \\ 0 & -2.179994 & -1.324933 & 0.135098 \\ 0 & -0.628378 & -0.133150 & -2.016455\end{array}$




$\begin{array}{lrrr}\mathrm{O} & -3.582339 & 0.325772 & 2.258566 \\ \mathrm{O} & -1.967306 & 1.556903 & -0.024969 \\ \mathrm{O} & -0.425475 & 2.874948 & -2.295800 \\ \mathrm{~V} & 0.774990 & -0.028959 & 0.915151 \\ \mathrm{O} & -0.861716 & -3.150958 & -1.922952 \\ \mathrm{C} & 3.995548 & 0.863249 & 0.390751 \\ \mathrm{O} & 5.187869 & 0.795747 & 0.260842 \\ \mathrm{O} & 3.189131 & -0.216191 & 0.615576 \\ \mathrm{H} & 3.380980 & 1.774114 & 0.344944 \\ \mathrm{C} & 3.866802 & -1.506539 & 0.678061 \\ \mathrm{H} & 3.070272 & -2.243332 & 0.791735 \\ \mathrm{H} & 4.422014 & -1.666305 & -0.251273 \\ \mathrm{H} & 4.554491 & -1.513169 & 1.529791 \\ \mathrm{TI} & -2.157065 & 0.185389 & 1.150157 \\ \mathrm{TI} & -0.733340 & -1.618790 & -0.964560 \\ \mathrm{TI} & -0.502387 & 1.482618 & -1.142761 \\ \mathrm{H} & -0.197177 & 3.808719 & -2.301897 \\ \mathrm{H} & -0.988971 & -3.401121 & -2.842786 \\ \mathrm{H} & -4.092226 & 1.025201 & 2.677525\end{array}$

11

$\begin{array}{lrrr}\mathrm{O} & 0.038935 & 1.765416 & 1.417768 \\ \mathrm{O} & 1.463889 & -0.840928 & 1.480064 \\ \mathrm{O} & -1.494520 & -0.779224 & 1.483771 \\ \mathrm{O} & 1.474417 & 0.768855 & -0.978903 \\ \mathrm{O} & -1.441841 & 0.826950 & -0.978121 \\ \mathrm{O} & 2.991438 & -1.872149 & -0.963702 \\ \mathrm{O} & -0.037112 & -1.707587 & -0.920300 \\ \mathrm{O} & -3.069983 & -1.745382 & -0.958148 \\ \mathrm{~V} & 0.003005 & 0.052870 & 1.831010 \\ \mathrm{O} & 0.069421 & 3.464116 & -1.086181 \\ \mathrm{TI} & 0.036469 & 1.780570 & -0.439310 \\ \mathrm{TI} & -1.585122 & -0.889491 & -0.388083 \\ \mathrm{TI} & 1.544742 & -0.954538 & -0.391829 \\ \mathrm{H} & -3.961060 & -1.533819 & -1.251503 \\ \mathrm{H} & 3.902525 & -1.703361 & -1.221064 \\ \mathrm{H} & 0.118281 & 4.376741 & -0.787760\end{array}$

\section{2}

$\begin{array}{rrrr}0 & -0.885132 & -1.165285 & 1.416844 \\ 0 & -1.584561 & 1.231879 & 0.000000 \\ 0 & 0.883324 & 1.068079 & 1.438941 \\ 0 & -0.885132 & -1.165285 & -1.416844 \\ 0 & 1.567503 & -1.298296 & 0.000000 \\ 0 & -1.735127 & 1.312092 & -3.053427 \\ 0 & 0.883324 & 1.068079 & -1.438941 \\ 0 & 3.596272 & 1.032177 & 0.000000\end{array}$




$\begin{array}{lrrr}\mathrm{V} & -0.122387 & -1.912423 & 0.000000 \\ \mathrm{O} & -1.735127 & 1.312092 & 3.053427 \\ \mathrm{O} & -0.217413 & -3.485584 & 0.000000 \\ \mathrm{TI} & -0.885132 & 0.679062 & -1.594703 \\ \mathrm{TI} & 1.874420 & 0.498470 & 0.000000 \\ \mathrm{TI} & -0.885132 & 0.679062 & 1.594703 \\ \mathrm{H} & 4.476163 & 0.643005 & 0.000000 \\ \mathrm{H} & -1.528074 & 1.609049 & -3.944644 \\ \mathrm{H} & -1.528074 & 1.609049 & 3.944644 .\end{array}$

For Figure 6: 1-4

1

$\begin{array}{lrrr}\mathrm{O} & -0.232613 & 0.001115 & 1.533887 \\ \mathrm{O} & -0.323968 & -1.383329 & -0.732639 \\ \mathrm{O} & -0.323920 & 1.382560 & -0.734449 \\ \mathrm{O} & 2.063483 & -1.457080 & 0.813029 \\ \mathrm{O} & 2.063605 & 1.457983 & 0.811270 \\ \mathrm{O} & 2.110401 & -3.046496 & -1.790670 \\ \mathrm{O} & 2.047421 & -0.001070 & -1.642164 \\ \mathrm{O} & 2.110468 & 3.044344 & -1.794197 \\ \mathrm{~V} & -1.119518 & 0.000068 & -0.094390 \\ \mathrm{O} & 2.056816 & 0.001938 & 3.552290 \\ \mathrm{TI} & 1.516721 & 0.001046 & 1.835487 \\ \mathrm{TI} & 1.556113 & 1.591812 & -0.899212 \\ \mathrm{TI} & 1.555982 & -1.592997 & -0.897331 \\ \mathrm{H} & 2.519773 & 3.897279 & -1.614512 \\ \mathrm{H} & 2.516844 & -3.900605 & -1.610102 \\ \mathrm{H} & 1.642436 & 0.004119 & 4.421820 \\ \mathrm{~S} & -3.803746 & 0.000021 & 0.018252 \\ \mathrm{O} & -4.531153 & -1.275708 & -0.003278 \\ \mathrm{O} & -2.619613 & 0.000921 & 1.126475 \\ \mathrm{O} & -4.531415 & 1.275570 & -0.005082 \\ \mathrm{O} & -2.627534 & -0.000702 & -1.156354\end{array}$

2

$\begin{array}{lrrr}\mathrm{O} & 0.122668 & 0.203459 & 1.502225 \\ \mathrm{O} & 0.071344 & 1.110498 & -1.069125 \\ \mathrm{O} & 0.105626 & -1.481324 & -0.551403 \\ \mathrm{O} & -2.223470 & 1.486579 & 0.571033 \\ \mathrm{O} & -2.192779 & -1.358802 & 1.107867 \\ \mathrm{O} & -2.371497 & 2.594829 & -2.279154 \\ \mathrm{O} & -2.301584 & -0.391072 & -1.594473 \\ \mathrm{O} & -2.286138 & -3.410418 & -1.170012 \\ \mathrm{~V} & 0.960342 & -0.055435 & -0.101425 \\ \mathrm{O} & -2.151572 & 0.571810 & 3.521759 \\ \mathrm{TI} & -1.633180 & 0.255708 & 1.825652\end{array}$




$\begin{array}{lrrr}\text { TI } & -1.754178 & -1.798595 & -0.579208 \\ \text { TI } & -1.782170 & 1.310986 & -1.163898 \\ \mathrm{H} & -2.598569 & -4.245514 & -0.806740 \\ \mathrm{H} & -2.875581 & 3.413885 & -2.245214 \\ \mathrm{H} & -1.727688 & 0.689877 & 4.378261 \\ \mathrm{~S} & 3.559131 & -0.734823 & -0.035104 \\ \mathrm{O} & 4.484118 & 0.392182 & 0.255646 \\ \mathrm{O} & 2.408467 & -0.786257 & 1.065985 \\ \mathrm{O} & 4.116328 & -2.043635 & -0.390560 \\ \mathrm{O} & 2.472341 & -0.228662 & -1.176019 \\ \mathrm{C} & 2.233371 & 3.020394 & -0.354586 \\ \mathrm{H} & 2.669289 & 2.686441 & -1.305808 \\ \mathrm{H} & 1.236024 & 3.431846 & -0.531786 \\ \mathrm{H} & 2.870151 & 3.792090 & 0.097154 \\ \mathrm{O} & 2.088029 & 1.920416 & 0.561542 \\ \mathrm{H} & 2.986771 & 1.606166 & 0.777553\end{array}$

3

$\begin{array}{lrrr}\mathrm{O} & 0.000180 & 1.537218 & -0.085173 \\ \mathrm{O} & 0.107481 & -0.495077 & 1.613124 \\ \mathrm{O} & -0.127123 & -0.786390 & -1.200959 \\ \mathrm{O} & 2.434697 & 1.113386 & 1.235599 \\ \mathrm{O} & 2.181388 & 0.795017 & -1.652971 \\ \mathrm{O} & 2.740651 & -1.298995 & 3.086245 \\ \mathrm{O} & 2.357657 & -1.493501 & 0.073715 \\ \mathrm{O} & 2.122161 & -1.991375 & -2.939725 \\ \mathrm{~V} & -0.853953 & -0.078726 & 0.246239 \\ \mathrm{O} & 2.148925 & 3.676711 & -0.508586 \\ \mathrm{TI} & 1.719170 & 1.941768 & -0.273754 \\ \mathrm{TI} & 1.698112 & -0.933334 & -1.545089 \\ \mathrm{TI} & 2.005835 & -0.582554 & 1.609039 \\ \mathrm{H} & 2.425435 & -1.891005 & -3.847789 \\ \mathrm{H} & 3.235965 & -0.996301 & 3.854480 \\ \mathrm{H} & 1.684373 & 4.515568 & -0.596072 \\ \mathrm{~S} & -3.990110 & 0.563813 & -0.033568 \\ \mathrm{O} & -4.788742 & 1.494904 & 0.769086 \\ \mathrm{O} & -2.369062 & 0.725071 & 0.596580 \\ \mathrm{O} & -3.843243 & 0.757185 & -1.487663 \\ \mathrm{O} & -4.215237 & -0.924652 & 0.370279 \\ \mathrm{C} & -1.626600 & -3.006865 & -0.146575 \\ \mathrm{H} & -0.837294 & -3.426411 & 0.492252 \\ \mathrm{H} & -2.521797 & -3.637485 & -0.079487 \\ \mathrm{H} & -1.278338 & -2.971952 & -1.188250 \\ \mathrm{O} & -1.955959 & -1.697000 & 0.305054 \\ \mathrm{H} & -3.124826 & -1.430893 & 0.336718\end{array}$




\begin{tabular}{|c|c|c|c|}
\hline 0 & 0.167410 & 1.439285 & -0.053277 \\
\hline 0 & 0.056137 & -0.892860 & -1.167731 \\
\hline 0 & -0.168381 & -0.680040 & 1.574531 \\
\hline 0 & -2.065116 & 0.933580 & -1.645967 \\
\hline O & -2.307898 & 1.213368 & 1.252909 \\
\hline 0 & -2.279655 & -1.801882 & -2.995847 \\
\hline 0 & -2.519356 & -1.354745 & 0.026612 \\
\hline 0 & -2.894138 & -1.173000 & 3.044300 \\
\hline V & 0.873561 & -0.309650 & 0.269179 \\
\hline 0 & -1.714144 & 3.788553 & -0.430895 \\
\hline $\mathrm{TI}$ & -1.478672 & 2.007407 & -0.234504 \\
\hline TI & -2.075875 & -0.519140 & 1.579804 \\
\hline $\mathrm{TI}$ & -1.774398 & -0.826626 & -1.566303 \\
\hline $\mathrm{H}$ & -3.377705 & -0.812143 & 3.794901 \\
\hline $\mathrm{H}$ & -2.590001 & -1.628630 & -3.890632 \\
\hline $\mathrm{H}$ & -1.123486 & 4.547429 & -0.486350 \\
\hline S & 3.830824 & 0.570543 & -0.043795 \\
\hline 0 & 3.505410 & 0.490908 & -1.481830 \\
\hline O & 2.417037 & 0.577887 & 0.772393 \\
\hline O & 4.695609 & 1.621048 & 0.492653 \\
\hline O & 4.434574 & -0.854532 & 0.473660 \\
\hline $\mathrm{C}$ & 1.518294 & -3.120528 & -0.132630 \\
\hline $\mathrm{H}$ & 0.784227 & -3.528852 & 0.579148 \\
\hline $\mathrm{H}$ & 1.068642 & -3.081868 & -1.136481 \\
\hline $\mathrm{H}$ & 2.405005 & -3.767711 & -0.153163 \\
\hline 0 & 1.910212 & -1.818074 & 0.269830 \\
\hline $\mathrm{H}$ & 3.673630 & -1.483858 & 0.409299 \\
\hline \multicolumn{4}{|c|}{ 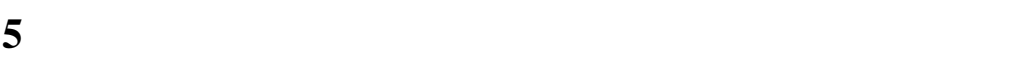 } \\
\hline O & 0.324118 & 0.321500 & 1.291637 \\
\hline 0 & -0.008154 & -0.306241 & -1.595635 \\
\hline O & -0.991258 & -1.809989 & 0.376632 \\
\hline O & -1.363977 & 1.964002 & -0.417503 \\
\hline O & -2.485370 & 0.273869 & 1.584666 \\
\hline O & -1.758185 & 1.459803 & -3.433650 \\
\hline O & -2.821940 & -0.349067 & -1.217022 \\
\hline O & -3.911436 & -2.407522 & 0.855978 \\
\hline V & 0.539999 & -0.840796 & -0.012027 \\
\hline O & -1.048349 & 2.807416 & 2.521681 \\
\hline $\mathrm{TI}$ & -1.151219 & 1.446213 & 1.340085 \\
\hline $\mathrm{TI}$ & -2.662900 & -1.166542 & 0.414728 \\
\hline $\mathrm{TI}$ & -1.504922 & 0.777245 & -1.781316 \\
\hline $\mathrm{H}$ & -4.840893 & -2.559494 & 0.660499 \\
\hline $\mathrm{H}$ & -1.661291 & 2.310612 & -3.872384 \\
\hline $\mathrm{H}$ & -1.434969 & 3.035367 & 3.372729 \\
\hline S & 3.675283 & 0.678654 & 0.065849 \\
\hline O & 4.597148 & 1.609261 & -0.595858 \\
\hline O & 2.355066 & 0.461015 & -0.607560 \\
\hline
\end{tabular}




$\begin{array}{lrrr}\mathrm{O} & 3.747145 & 0.467987 & 1.521601 \\ \mathrm{O} & 4.504332 & -1.069280 & -0.554301 \\ \mathrm{C} & 2.708479 & -2.968579 & 0.110980 \\ \mathrm{H} & 2.706837 & -3.662557 & -0.736904 \\ \mathrm{H} & 3.865785 & -1.898007 & -0.268948 \\ \mathrm{H} & 2.999591 & -3.358693 & 1.092787 \\ \mathrm{O} & 1.681055 & -2.129772 & 0.140992 \\ \mathrm{H} & 5.346887 & -1.171929 & -0.080791 \\ \mathbf{6} & & & \\ \mathrm{O} & 0.329042 & -0.356412 & -1.295443 \\ \mathrm{O} & -0.012375 & 0.279822 & 1.624658 \\ \mathrm{O} & -1.018483 & 1.828321 & -0.381352 \\ \mathrm{O} & -1.380518 & -1.969547 & 0.416262 \\ \mathrm{O} & -2.497741 & -0.281935 & -1.584943 \\ \mathrm{O} & -1.789123 & -1.487439 & 3.434078 \\ \mathrm{O} & -2.834331 & 0.337938 & 1.219377 \\ \mathrm{O} & -3.924318 & 2.415370 & -0.849892 \\ \mathrm{~V} & 0.518660 & 0.810249 & 0.027511 \\ \mathrm{O} & -1.058762 & -2.816751 & -2.528491 \\ \mathrm{TI} & -1.161944 & -1.448970 & -1.343331 \\ \mathrm{TI} & -2.664648 & 1.169873 & -0.416370 \\ \mathrm{TI} & -1.524111 & -0.785516 & 1.784997 \\ \mathrm{H} & -4.883374 & 2.432336 & -0.773772 \\ \mathrm{H} & -1.774738 & -2.363904 & 3.829964 \\ \mathrm{H} & -1.532812 & -3.070838 & -3.326155 \\ \mathrm{~S} & 3.723297 & -0.755421 & -0.103551 \\ \mathrm{O} & 4.708748 & -1.632946 & 0.534178 \\ \mathrm{O} & 2.446716 & -0.505319 & 0.611149 \\ \mathrm{O} & 3.828390 & -0.412985 & -1.528473 \\ \mathrm{O} & 4.667297 & 1.188840 & 0.661622 \\ \mathrm{C} & 2.433573 & 3.184604 & -0.184014 \\ \mathrm{H} & 4.084439 & 1.930417 & 0.384371 \\ \mathrm{O} & 1.638681 & 2.188648 & -0.118912 \\ \mathrm{H} & 5.513738 & 1.329870 & 0.212240 \\ \mathrm{H} & 2.830055 & 3.476895 & -1.160143 \\ \mathrm{H} & 2.549024 & 3.825257 & 0.694565\end{array}$

\section{For Figure S3: 2-8}

2

$\begin{array}{rrrr}0 & -0.490860 & 1.647806 & -0.389881 \\ 0 & 1.902330 & 0.495591 & -1.475042 \\ 0 & 1.710583 & 1.050747 & 1.322338 \\ 0 & -0.529083 & -1.022930 & -1.310871 \\ 0 & -0.695530 & -0.483744 & 1.456310 \\ 0 & 1.951386 & -2.339533 & -2.609858\end{array}$




$\begin{array}{lrrr}\mathrm{O} & 1.662544 & -1.666986 & 0.386332 \\ \mathrm{O} & 1.588068 & -1.185677 & 3.418719 \\ \mathrm{~V} & -1.271779 & 0.079559 & -0.143312 \\ \mathrm{O} & 2.022154 & 3.428740 & -0.617730 \\ \mathrm{O} & -2.850134 & 0.144635 & -0.257017 \\ \mathrm{TI} & 1.315581 & -1.228421 & -1.343909 \\ \mathrm{TI} & 1.096927 & -0.618914 & 1.782683 \\ \mathrm{TI} & 1.359034 & 1.785024 & -0.300248 \\ \mathrm{H} & 1.196275 & -1.568643 & 4.209778 \\ \mathrm{H} & 2.158765 & -3.271049 & -2.731547 \\ \mathrm{H} & 2.413160 & 4.166953 & -0.140634 \\ \mathrm{C} & -6.496770 & 0.503478 & -0.108075 \\ \mathrm{H} & -7.545966 & 0.218528 & 0.040228 \\ \mathrm{H} & -6.387868 & 0.911698 & -1.127476 \\ \mathrm{H} & -6.245443 & 1.295424 & 0.618318 \\ \mathrm{O} & -5.715012 & -0.667790 & 0.085548 \\ \mathrm{H} & -4.782873 & -0.439712 & -0.042399\end{array}$

3

$\begin{array}{lrrr}0 & -0.447240 & -1.242442 & 1.319434 \\ \mathrm{O} & -0.215264 & -1.141926 & -1.431752 \\ \mathrm{O} & -1.337894 & 0.915297 & -0.189833 \\ \mathrm{O} & 2.160989 & -0.884945 & 0.140911 \\ \mathrm{O} & 0.904104 & 1.279758 & 1.517234 \\ \mathrm{O} & 2.490197 & -0.909986 & -2.933255 \\ \mathrm{O} & 1.175709 & 1.340387 & -1.374785 \\ \mathrm{O} & -0.229125 & 3.743921 & -0.020003 \\ \mathrm{~V} & -1.377256 & -0.984780 & -0.126454 \\ \mathrm{O} & -2.308198 & -2.381829 & -0.378182 \\ \mathrm{O} & 2.009433 & -1.001102 & 3.202083 \\ \mathrm{C} & -4.171351 & 0.537463 & -0.150974 \\ \mathrm{H} & -5.183130 & 0.367072 & 0.245386 \\ \mathrm{H} & -4.230812 & 0.634077 & -1.246602 \\ \mathrm{H} & -3.774072 & 1.470467 & 0.267919 \\ \mathrm{O} & -3.352133 & -0.553018 & 0.233354 \\ \mathrm{H} & -3.262208 & -1.733932 & -0.173132 \\ \mathrm{TI} & 1.506812 & -0.432126 & -1.494977 \\ \mathrm{TI} & 1.227848 & -0.470415 & 1.664508 \\ \mathrm{TI} & 0.078878 & 1.956492 & -0.020990 \\ \mathrm{H} & -0.999069 & 4.310398 & -0.128294 \\ \mathrm{H} & 2.259158 & -0.586939 & 4.034245 \\ \mathrm{H} & 3.192656 & -1.535831 & -3.134770\end{array}$

4

$\begin{array}{rrrr}0 & 1.281442 & 0.696626 & -0.932264 \\ 0 & -0.137841 & -1.437412 & -1.013550 \\ 0 & 0.715950 & -0.938100 & 1.450012\end{array}$




$\begin{array}{lrrr}\mathrm{O} & -1.493416 & 0.941408 & -1.490527 \\ \mathrm{O} & -0.538212 & 1.656151 & 1.111791 \\ \mathrm{O} & -3.006779 & -1.769538 & -1.948674 \\ \mathrm{O} & -2.093399 & -0.709995 & 0.853908 \\ \mathrm{O} & -1.247646 & 0.084110 & 3.649042 \\ \mathrm{~V} & 1.476522 & -0.879618 & -0.100105 \\ \mathrm{O} & 2.124143 & -2.413852 & -0.798520 \\ \mathrm{O} & 0.118174 & 3.520666 & -1.266066 \\ \mathrm{C} & 4.141452 & 0.360583 & -0.163417 \\ \mathrm{H} & 4.694210 & -0.245791 & -0.899472 \\ \mathrm{H} & 4.831480 & 0.682099 & 0.629139 \\ \mathrm{H} & 3.714826 & 1.240436 & -0.672127 \\ \mathrm{O} & 3.101506 & -0.390594 & 0.414974 \\ \mathrm{H} & 3.048481 & -2.575036 & -0.562897 \\ \mathrm{TI} & -1.776718 & -0.841897 & -0.982028 \\ \mathrm{TI} & -0.840901 & 0.053780 & 1.887306 \\ \mathrm{TI} & -0.161946 & 1.823141 & -0.694854 \\ \mathrm{H} & -0.293703 & 4.087739 & -1.925658 \\ \mathrm{H} & -2.953859 & -2.556976 & -2.499604 \\ \mathrm{H} & -1.291096 & 0.748961 & 4.343188\end{array}$

5

$\begin{array}{lrrr}\mathrm{O} & -0.854734 & -0.975750 & -1.163641 \\ \mathrm{O} & -0.492556 & 1.571647 & -0.627746 \\ \mathrm{O} & -0.942819 & -0.103177 & 1.564470 \\ \mathrm{O} & 1.737503 & 0.101820 & -1.565957 \\ \mathrm{O} & 1.296561 & -1.707524 & 0.608002 \\ \mathrm{O} & 1.962033 & 3.190211 & -1.139054 \\ \mathrm{O} & 1.661362 & 1.052787 & 1.162373 \\ \mathrm{O} & 1.385907 & -0.855450 & 3.522573 \\ \mathrm{~V} & -1.646701 & 0.150946 & 0.021520 \\ \mathrm{O} & -2.939321 & 1.607415 & -0.028250 \\ \mathrm{O} & 1.271831 & -2.847459 & -2.288069 \\ \mathrm{C} & -4.465791 & -0.361308 & -0.213754 \\ \mathrm{H} & -5.222916 & -0.633827 & 0.528648 \\ \mathrm{H} & -4.734136 & -0.500971 & -1.269348 \\ \mathrm{H} & -3.942828 & 1.120857 & -0.155183 \\ \mathrm{O} & -3.215368 & -0.706595 & 0.112453 \\ \mathrm{H} & -2.782143 & 2.413193 & -0.537947 \\ \mathrm{TI} & 1.266936 & 1.594967 & -0.590690 \\ \mathrm{TI} & 0.917636 & -0.429038 & 1.826649 \\ \mathrm{TI} & 0.895029 & -1.455229 & -1.186438 \\ \mathrm{H} & 1.900737 & -3.023282 & -2.993991 \\ \mathrm{H} & 2.853568 & 3.481287 & -1.353969 \\ \mathrm{H} & 1.882155 & -0.419987 & 4.222654\end{array}$




$\begin{array}{lrrr}\mathrm{O} & 0.650508 & 1.667648 & -0.008143 \\ \mathrm{O} & 0.903037 & -0.643245 & -1.535331 \\ \mathrm{O} & 0.906422 & -0.629954 & 1.539363 \\ \mathrm{O} & -1.592673 & 0.779941 & -1.472182 \\ \mathrm{O} & -1.590095 & 0.791827 & 1.467934 \\ \mathrm{O} & -1.485218 & -1.849325 & -3.052749 \\ \mathrm{O} & -1.327765 & -1.644153 & 0.007988 \\ \mathrm{O} & -1.481992 & -1.823394 & 3.069535 \\ \mathrm{~V} & 1.600445 & -0.042515 & -0.000347 \\ \mathrm{O} & 2.657557 & -2.051779 & 0.000702 \\ \mathrm{O} & -1.704702 & 3.552609 & -0.013637 \\ \mathrm{C} & 4.334087 & 1.357720 & -0.004682 \\ \mathrm{H} & 4.819099 & 1.605247 & 0.945344 \\ \mathrm{H} & 4.818347 & 1.597499 & -0.957071 \\ \mathrm{H} & 2.305569 & -2.508259 & 0.779181 \\ \mathrm{O} & 3.220161 & 0.787114 & -0.001811 \\ \mathrm{H} & 2.287441 & -2.509215 & -0.768881 \\ \mathrm{TI} & -1.080298 & 1.835014 & -0.007046 \\ \mathrm{TI} & -0.936198 & -0.877326 & -1.615164 \\ \mathrm{TI} & -0.933650 & -0.863899 & 1.623473 \\ \mathrm{H} & -1.236358 & 4.393283 & -0.018528 \\ \mathrm{H} & -2.075272 & -1.670856 & -3.791376 \\ \mathrm{H} & -2.072295 & -1.637856 & 3.806267\end{array}$

7

$\begin{array}{lrrr}\mathrm{O} & -0.922819 & 0.024003 & -1.933579 \\ \mathrm{O} & 1.122765 & -1.651487 & -0.730316 \\ \mathrm{O} & 1.321494 & 1.483843 & -0.764547 \\ \mathrm{O} & -1.477974 & -1.358720 & 0.590435 \\ \mathrm{O} & -1.307817 & 1.528360 & 0.567618 \\ \mathrm{O} & 0.676279 & -3.041096 & 1.927905 \\ \mathrm{O} & 0.839680 & -0.021448 & 1.765740 \\ \mathrm{O} & 1.053815 & 3.005757 & 1.824936 \\ \mathrm{~V} & 0.773679 & -0.073920 & -1.489675 \\ \mathrm{O} & 2.907797 & -0.168288 & -2.160739 \\ \mathrm{O} & -3.725182 & 0.237773 & -0.738915 \\ \mathrm{H} & 3.345800 & 0.588996 & -1.742006 \\ \mathrm{H} & 3.299705 & -0.968076 & -1.778425 \\ \mathrm{TI} & -1.951699 & 0.121376 & -0.388007 \\ \mathrm{TI} & 0.300321 & -1.568415 & 0.935801 \\ \mathrm{TI} & 0.505377 & 1.552436 & 0.887516 \\ \mathrm{H} & -4.343099 & -0.021139 & -1.428392 \\ \mathrm{H} & 0.247370 & -3.878134 & 2.128263 \\ \mathrm{H} & 0.963323 & 3.350224 & 2.717951\end{array}$

8

$\begin{array}{llll}0 & 0.038935 & 1.765416 & 1.417768\end{array}$ 


$\begin{array}{lrrr}\mathrm{O} & 1.463889 & -0.840928 & 1.480064 \\ \mathrm{O} & -1.494520 & -0.779224 & 1.483771 \\ \mathrm{O} & 1.474417 & 0.768855 & -0.978903 \\ \mathrm{O} & -1.441841 & 0.826950 & -0.978121 \\ \mathrm{O} & 2.991438 & -1.872149 & -0.963702 \\ \mathrm{O} & -0.037112 & -1.707587 & -0.920300 \\ \mathrm{O} & -3.069983 & -1.745382 & -0.958148 \\ \mathrm{~V} & 0.003005 & 0.052870 & 1.831010 \\ \mathrm{O} & 0.069421 & 3.464116 & -1.086181 \\ \mathrm{TI} & 0.036469 & 1.780570 & -0.439310 \\ \mathrm{TI} & -1.585122 & -0.889491 & -0.388083 \\ \mathrm{TI} & 1.544742 & -0.954538 & -0.391829 \\ \mathrm{H} & -3.961060 & -1.533819 & -1.251503 \\ \mathrm{H} & 3.902525 & -1.703361 & -1.221064 \\ \mathrm{H} & 0.118281 & 4.376741 & -0.787760\end{array}$

For Figure S4: 2-13

2

$\begin{array}{lr}\mathrm{O} & -0.490860 \\ \mathrm{O} & 1.902330 \\ \mathrm{O} & 1.710583 \\ \mathrm{O} & -0.529083 \\ \mathrm{O} & -0.695530 \\ \mathrm{O} & 1.951386 \\ \mathrm{O} & 1.662544 \\ \mathrm{O} & 1.588068 \\ \mathrm{~V} & -1.271779 \\ \mathrm{O} & 2.022154 \\ \mathrm{O} & -2.850134 \\ \mathrm{TI} & 1.315581 \\ \mathrm{TI} & 1.096927 \\ \mathrm{TI} & 1.359034 \\ \mathrm{H} & 1.196275 \\ \mathrm{H} & 2.158765 \\ \mathrm{H} & 2.413160 \\ \mathrm{C} & -6.496770 \\ \mathrm{H} & -7.545966 \\ \mathrm{H} & -6.387868 \\ \mathrm{H} & -6.245443 \\ \mathrm{O} & -5.715012 \\ \mathrm{H} & -4.782873 \\ \mathrm{H} & \end{array}$

$$
\begin{array}{rr}
1.647806 & -0.389881 \\
0.495591 & -1.475042 \\
1.050747 & 1.322338 \\
-1.022930 & -1.310871 \\
-0.483744 & 1.456310 \\
-2.339533 & -2.609858 \\
-1.666986 & 0.386332 \\
-1.185677 & 3.418719 \\
0.079559 & -0.143312 \\
3.428740 & -0.617730 \\
0.144635 & -0.257017 \\
-1.228421 & -1.343909 \\
-0.618914 & 1.782683 \\
1.785024 & -0.300248 \\
-1.568643 & 4.209778 \\
-3.271049 & -2.731547 \\
4.166953 & -0.140634 \\
0.503478 & -0.108075 \\
0.218528 & 0.040228 \\
0.911698 & -1.127476 \\
1.295424 & 0.618318 \\
-0.667790 & 0.085548 \\
-0.439712 & -0.042399
\end{array}
$$

3

$\begin{array}{rrrr}0 & -0.447240 & -1.242442 & 1.319434 \\ 0 & -0.215264 & -1.141926 & -1.431752 \\ 0 & -1.337894 & 0.915297 & -0.189833\end{array}$




$\begin{array}{lrrr}\mathrm{O} & 2.160989 & -0.884945 & 0.140911 \\ \mathrm{O} & 0.904104 & 1.279758 & 1.517234 \\ \mathrm{O} & 2.490197 & -0.909986 & -2.933255 \\ \mathrm{O} & 1.175709 & 1.340387 & -1.374785 \\ \mathrm{O} & -0.229125 & 3.743921 & -0.020003 \\ \mathrm{~V} & -1.377256 & -0.984780 & -0.126454 \\ \mathrm{O} & -2.308198 & -2.381829 & -0.378182 \\ \mathrm{O} & 2.009433 & -1.001102 & 3.202083 \\ \mathrm{C} & -4.171351 & 0.537463 & -0.150974 \\ \mathrm{H} & -5.183130 & 0.367072 & 0.245386 \\ \mathrm{H} & -4.230812 & 0.634077 & -1.246602 \\ \mathrm{H} & -3.774072 & 1.470467 & 0.267919 \\ \mathrm{O} & -3.352133 & -0.553018 & 0.233354 \\ \mathrm{H} & -3.262208 & -1.733932 & -0.173132 \\ \mathrm{TI} & 1.506812 & -0.432126 & -1.494977 \\ \mathrm{TI} & 1.227848 & -0.470415 & 1.664508 \\ \mathrm{TI} & 0.078878 & 1.956492 & -0.020990 \\ \mathrm{H} & -0.999069 & 4.310398 & -0.128294 \\ \mathrm{H} & 2.259158 & -0.586939 & 4.034245 \\ \mathrm{H} & 3.192656 & -1.535831 & -3.134770\end{array}$

4

$\begin{array}{lrrr}\mathrm{O} & 1.281442 & 0.696626 & -0.932264 \\ \mathrm{O} & -0.137841 & -1.437412 & -1.013550 \\ \mathrm{O} & 0.715950 & -0.938100 & 1.450012 \\ \mathrm{O} & -1.493416 & 0.941408 & -1.490527 \\ \mathrm{O} & -0.538212 & 1.656151 & 1.111791 \\ \mathrm{O} & -3.006779 & -1.769538 & -1.948674 \\ \mathrm{O} & -2.093399 & -0.709995 & 0.853908 \\ \mathrm{O} & -1.247646 & 0.084110 & 3.649042 \\ \mathrm{~V} & 1.476522 & -0.879618 & -0.100105 \\ \mathrm{O} & 2.124143 & -2.413852 & -0.798520 \\ \mathrm{O} & 0.118174 & 3.520666 & -1.266066 \\ \mathrm{C} & 4.141452 & 0.360583 & -0.163417 \\ \mathrm{H} & 4.694210 & -0.245791 & -0.899472 \\ \mathrm{H} & 4.831480 & 0.682099 & 0.629139 \\ \mathrm{H} & 3.714826 & 1.240436 & -0.672127 \\ \mathrm{O} & 3.101506 & -0.390594 & 0.414974 \\ \mathrm{H} & 3.048481 & -2.575036 & -0.562897 \\ \mathrm{TI} & -1.776718 & -0.841897 & -0.982028 \\ \mathrm{TI} & -0.840901 & 0.053780 & 1.887306 \\ \mathrm{TI} & -0.161946 & 1.823141 & -0.694854 \\ \mathrm{H} & -0.293703 & 4.087739 & -1.925658 \\ \mathrm{H} & -2.953859 & -2.556976 & -2.499604 \\ \mathrm{H} & -1.291096 & 0.748961 & 4.343188\end{array}$




\begin{tabular}{|c|c|c|c|}
\hline 0 & 0.285091 & 1.531507 & -0.669759 \\
\hline 0 & -0.125283 & -0.883515 & -1.437916 \\
\hline 0 & 0.834053 & -0.870187 & 1.025097 \\
\hline 0 & -2.398696 & 0.668796 & -1.074040 \\
\hline 0 & -1.408732 & 0.890037 & 1.601435 \\
\hline 0 & -2.681190 & -2.153899 & -2.441494 \\
\hline 0 & -1.882502 & -1.714433 & 0.566886 \\
\hline 0 & -1.003846 & -1.513914 & 3.460246 \\
\hline V & 1.249674 & 0.048391 & -0.384235 \\
\hline O & 2.379705 & -0.656501 & -1.554538 \\
\hline 0 & -1.988261 & 3.483643 & 0.011035 \\
\hline $\mathrm{C}$ & 3.109961 & 2.256363 & 0.018571 \\
\hline $\mathrm{H}$ & 3.774151 & 2.130220 & -0.851322 \\
\hline $\mathrm{H}$ & 3.694381 & 2.635552 & 0.868602 \\
\hline $\mathrm{H}$ & 2.313990 & 2.974188 & -0.237605 \\
\hline 0 & 2.528991 & 1.023341 & 0.376752 \\
\hline $\mathrm{H}$ & 3.336312 & -0.563356 & -1.351797 \\
\hline TI & -1.827987 & -1.118354 & -1.208173 \\
\hline TI & -0.905045 & -0.833691 & 1.783228 \\
\hline TI & -1.436957 & 1.755803 & -0.040738 \\
\hline $\mathrm{H}$ & -2.697244 & 3.970322 & -0.421175 \\
\hline $\mathrm{H}$ & -2.368463 & -2.607010 & -3.23 \\
\hline $\mathrm{H}$ & -1.267390 & -1.175869 & 4.321631 \\
\hline $\mathrm{C}$ & 5.089016 & -1.227063 & 0.422698 \\
\hline 0 & 5.061223 & -0.731213 & -0.683930 \\
\hline $\mathrm{H}$ & 6.053240 & -1.445510 & 0.926393 \\
\hline $\mathrm{H}$ & 4.159977 & -1.479313 & 0.972844 \\
\hline & & & \\
\hline 0 & 0.534582 & 1.409220 & -0.759363 \\
\hline 0 & -0.379010 & -0.870579 & -1.548487 \\
\hline 0 & 0.792278 & -1.226055 & 0.762016 \\
\hline O & -2.268790 & 1.053584 & -0.841442 \\
\hline O & -0.944464 & 0.913778 & 1.692421 \\
\hline 0 & -3.270366 & -1.556039 & -2.244272 \\
\hline 0 & -2.036120 & -1.479106 & 0.612468 \\
\hline 0 & -0.803719 & -1.643710 & 3.3 \\
\hline V & 1.172666 & -0.256215 & -0.645591 \\
\hline 0 & 2.250754 & -0.878848 & -1.787269 \\
\hline 0 & -1.226589 & 3.676820 & 0.341456 \\
\hline $\mathrm{C}$ & 3.273100 & 1.737570 & 0.082218 \\
\hline $\mathrm{H}$ & 3.896842 & 1.650848 & -0.821420 \\
\hline $\mathrm{H}$ & 3.901882 & 2.049077 & 0.929492 \\
\hline $\mathrm{H}$ & 2.498581 & 2.494983 & -0.093204 \\
\hline 0 & 2.657213 & 0.495416 & 0.401531 \\
\hline $\mathrm{H}$ & 3.302553 & -1.032005 & -1.505075 \\
\hline TI & -2.084310 & -0.786792 & -1.106427 \\
\hline
\end{tabular}




\begin{tabular}{|c|c|c|c|}
\hline $\mathrm{TI}$ & -0.768320 & -0.885459 & 1.751784 \\
\hline TI & -1.016641 & 1.892228 & 0.123138 \\
\hline $\mathrm{H}$ & -1.829117 & 4.346081 & 0.002071 \\
\hline $\mathrm{H}$ & -3.201340 & -2.033189 & -3.077094 \\
\hline $\mathrm{H}$ & -0.920553 & -1.350836 & 4.302015 \\
\hline $\mathrm{C}$ & 4.098898 & -0.872088 & 0.375716 \\
\hline O & 4.461959 & -0.934341 & -0.826937 \\
\hline $\mathrm{H}$ & 4.739810 & -0.345285 & $1.09811 \mathrm{~s}$ \\
\hline $\mathrm{H}$ & 3.390187 & -1.600227 & 0.797080 \\
\hline \multicolumn{4}{|c|}{7} \\
\hline O & 0.609421 & 0.852772 & -1.153181 \\
\hline 0 & -1.025395 & -1.418331 & -1.40375 \\
\hline 0 & 0.596871 & -1.157066 & 0.87268 \\
\hline 0 & -2.208821 & 1.133038 & -0.91042 \\
\hline 0 & -0.565062 & 1.404721 & 1.416056 \\
\hline 0 & -4.114076 & -1.258634 & -1.26733 \\
\hline 0 & -2.236428 & -0.896230 & 1.12268 \\
\hline 0 & -0.585722 & -0.678682 & 3.68869 \\
\hline V & 0.604491 & -0.897622 & -0.8750 \\
\hline 0 & 1.765653 & -1.674958 & -1.62550 \\
\hline 0 & -0.501057 & 3.680438 & -0.64395 \\
\hline $\mathrm{C}$ & 4.208139 & 1.503357 & -0.03033 \\
\hline $\mathrm{H}$ & 4.413839 & 1.422341 & -1.10682 \\
\hline $\mathrm{H}$ & 5.154129 & 1.698282 & 0.5036 \\
\hline $\mathrm{H}$ & 3.515096 & 2.332701 & 0.1491 \\
\hline 0 & 3.581482 & 0.325451 & 0.4714 \\
\hline $\mathrm{H}$ & 3.788572 & -1.492810 & -1.36494 \\
\hline $\mathrm{TI}$ & -2.521458 & -0.668900 & -0.66563 \\
\hline TI & -0.689568 & -0.332537 & $1.9226^{\circ}$ \\
\hline $\mathrm{TI}$ & -0.675207 & 1.910535 & -0.34717 \\
\hline $\mathrm{H}$ & -0.944702 & 4.377331 & -1.13702 \\
\hline $\mathrm{H}$ & -4.471825 & -1.920653 & -1.86666 \\
\hline $\mathrm{H}$ & -0.210261 & -0.276174 & 4.47791 \\
\hline $\mathrm{C}$ & 4.383284 & -0.834936 & 0.3574 \\
\hline 0 & 4.632182 & -1.216574 & -0.97133 \\
\hline $\mathrm{H}$ & 5.373141 & -0.663929 & 0.8108 \\
\hline $\mathrm{H}$ & 3.834910 & -1.610038 & 0.9115 \\
\hline \multicolumn{4}{|c|}{ 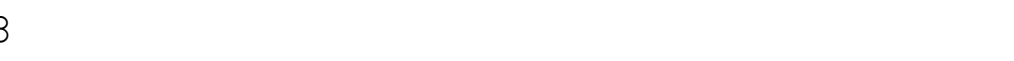 } \\
\hline O & 0.507587 & -1.918327 & -0.1522 \\
\hline O & 2.558171 & -0.152811 & 0.8515 \\
\hline O & 1.641851 & 0.105964 & -1.83738 \\
\hline 0 & -0.184185 & -0.004950 & 1.712 \\
\hline O & -0.989879 & 0.289797 & -0.8158 \\
\hline O & 1.893791 & 1.877956 & 3.0 \\
\hline O & 0.932438 & 2.099640 & 0.1 \\
\hline
\end{tabular}




$\begin{array}{lrrr}\mathrm{O} & -0.144465 & 2.492605 & -2.729318 \\ \mathrm{~V} & -0.855463 & -1.076315 & 0.508767 \\ \mathrm{O} & 3.429849 & -2.272667 & -1.170462 \\ \mathrm{O} & -1.674881 & -2.203758 & 1.469003 \\ \mathrm{TI} & 1.355306 & 1.041378 & 1.545895 \\ \mathrm{TI} & 0.299415 & 1.328248 & -1.411275 \\ \mathrm{TI} & 2.145650 & -1.123196 & -0.635603 \\ \mathrm{H} & -0.942730 & 2.700540 & -3.224318 \\ \mathrm{H} & 1.937056 & 2.783989 & 3.373974 \\ \mathrm{H} & 3.889738 & -2.475586 & -1.991165 \\ \mathrm{C} & -3.705877 & -0.644831 & -0.693304 \\ \mathrm{H} & -3.358193 & 0.038431 & -1.487643 \\ \mathrm{H} & -4.596456 & -1.202053 & -1.042192 \\ \mathrm{C} & -5.013299 & 1.090603 & 0.236132 \\ \mathrm{H} & -5.962135 & 0.645441 & -0.111157 \\ \mathrm{H} & -5.187152 & 1.603140 & 1.188372 \\ \mathrm{H} & -4.670505 & 1.825811 & -0.513254 \\ \mathrm{O} & -4.029677 & 0.097188 & 0.473178 \\ \mathrm{O} & -2.708622 & -1.552255 & -0.375312 \\ \mathrm{H} & -2.582918 & -2.127648 & 0.741181\end{array}$

$\begin{array}{lrrr}\mathrm{O} & 0.179348 & -1.532138 & 0.735020 \\ \mathrm{O} & -2.540329 & -1.137193 & -0.051896 \\ \mathrm{O} & -1.387180 & 0.660815 & 1.831533 \\ \mathrm{O} & -0.330021 & -0.541435 & -1.671133 \\ \mathrm{O} & 0.804727 & 1.152401 & -0.007543 \\ \mathrm{O} & -3.009683 & -0.279820 & -3.038276 \\ \mathrm{O} & -1.888504 & 1.620192 & -0.806786 \\ \mathrm{O} & -0.721381 & 3.584017 & 1.199476 \\ \mathrm{~V} & 1.156146 & -0.527504 & -0.287459 \\ \mathrm{O} & -2.092189 & -2.163401 & 2.773219 \\ \mathrm{O} & 2.056162 & -1.156648 & -1.727195 \\ \mathrm{TI} & -2.002235 & -0.108004 & -1.531953 \\ \mathrm{TI} & -0.817577 & 1.882875 & 0.593839 \\ \mathrm{TI} & -1.530510 & -1.111267 & 1.413432 \\ \mathrm{H} & -1.040541 & 4.438207 & 0.891586 \\ \mathrm{H} & -2.821968 & -0.564095 & -3.938011 \\ \mathrm{H} & -2.799796 & -2.805227 & 2.890548 \\ \mathrm{C} & 4.040134 & -0.775272 & 0.247043 \\ \mathrm{H} & 4.537869 & -1.318434 & 1.072467 \\ \mathrm{H} & 4.134492 & -1.340669 & -0.699150 \\ \mathrm{C} & 5.955553 & 0.490591 & -0.231513 \\ \mathrm{H} & 6.118839 & -0.014291 & -1.199378 \\ \mathrm{H} & 6.281817 & 1.532905 & -0.308808 \\ \mathrm{H} & 6.554463 & -0.018827 & 0.543787 \\ \mathrm{O} & 4.577449 & 0.518939 & 0.111969\end{array}$




\begin{tabular}{|c|c|c|c|}
\hline 0 & 2.692473 & -0.658279 & 0.562755 \\
\hline $\mathrm{H}$ & 1.449472 & -1.207788 & -2.480824 \\
\hline \\
\hline O & 0.324259 & -0.674181 & -1.370884 \\
\hline 0 & -2.315449 & 0.319162 & -1.462615 \\
\hline 0 & -1.657482 & -1.851739 & 0.324157 \\
\hline 0 & -0.118913 & 1.695252 & -0.361613 \\
\hline 0 & 0.558666 & -0.355785 & 1.450517 \\
\hline O & -2.764530 & 3.197892 & -0.427512 \\
\hline 0 & -2.130630 & 0.735707 & 1.403294 \\
\hline 0 & -1.658633 & -1.519085 & 3.359368 \\
\hline V & 1.142177 & 0.276600 & -0.015160 \\
\hline O & -1.790801 & -2.406696 & -2.750148 \\
\hline O & 2.334646 & 1.767595 & 0.264576 \\
\hline C & 3.971762 & 0.048549 & -0.471603 \\
\hline $\mathrm{H}$ & 4.258879 & 0.169457 & -1.532459 \\
\hline $\mathrm{H}$ & 3.389757 & 1.367253 & -0.052216 \\
\hline C & 6.278643 & -0.024805 & -0.027454 \\
\hline $\mathrm{H}$ & 6.376833 & 1.066007 & 0.092022 \\
\hline $\mathrm{H}$ & 6.969015 & -0.530351 & 0.654176 \\
\hline $\mathrm{H}$ & 6.520140 & -0.302389 & -1.066416 \\
\hline 0 & 4.969358 & -0.462824 & 0.327606 \\
\hline O & 2.784889 & -0.488229 & -0.250231 \\
\hline $\mathrm{H}$ & 2.112870 & 2.652295 & -0.054312 \\
\hline TI & -1.374654 & -1.233515 & -1.424874 \\
\hline $\mathrm{TI}$ & -1.291090 & -0.812916 & 1.731897 \\
\hline TI & -1.877598 & 1.617327 & -0.230217 \\
\hline $\mathrm{H}$ & -2.506293 & 4.094666 & -0.658539 \\
\hline $\mathrm{H}$ & -1.299036 & -2.846517 & -3.449879 \\
\hline $\mathrm{H}$ & -1.924159 & -2.390975 & 3.668789 \\
\hline
\end{tabular}

11

$\begin{array}{rrrr}\mathrm{O} & 1.038848 & -0.480408 & -0.929988 \\ \mathrm{O} & -1.707228 & -0.806868 & -1.678890 \\ \mathrm{O} & -0.725983 & -1.994085 & 0.785817 \\ \mathrm{O} & -0.817181 & 1.775773 & -0.835390 \\ \mathrm{O} & 0.280863 & 0.569482 & 1.704426 \\ \mathrm{O} & -3.725205 & 1.527162 & -1.634855 \\ \mathrm{O} & -2.515261 & 0.219986 & 0.910890 \\ \mathrm{O} & -1.649498 & -1.057743 & 3.531304 \\ \mathrm{~V} & 0.751437 & 1.124416 & 0.143939 \\ \mathrm{O} & 0.275092 & -3.181490 & -1.942268 \\ \mathrm{O} & 0.934321 & 3.222384 & 0.168327 \\ \mathrm{C} & 3.543810 & 0.288911 & -0.085388 \\ \mathrm{H} & 3.092903 & -0.710755 & -0.160342 \\ \mathrm{H} & 1.747050 & 3.696145 & -0.046918\end{array}$




$\begin{array}{lrrr}\mathrm{C} & 5.617703 & -0.853452 & -0.011492 \\ \mathrm{H} & 4.964812 & -1.714334 & -0.200979 \\ \mathrm{H} & 6.371506 & -0.770599 & -0.800297 \\ \mathrm{H} & 6.099335 & -0.946866 & 0.967698 \\ \mathrm{O} & 4.857303 & 0.378867 & -0.018298 \\ \mathrm{O} & 2.853888 & 1.309788 & -0.082005 \\ \mathrm{H} & 0.202832 & 3.518326 & -0.403525 \\ \mathrm{TI} & -2.257356 & 0.752282 & -0.863325 \\ \mathrm{TI} & -1.204848 & -0.618692 & 1.817808 \\ \mathrm{TI} & -0.254662 & -1.681267 & -1.020310 \\ \mathrm{H} & -1.643063 & -1.892633 & 4.009133 \\ \mathrm{H} & -3.859388 & 2.370737 & -2.075811 \\ \mathrm{H} & -0.256756 & -3.838232 & -2.402643\end{array}$

12

$\begin{array}{lrrr}\mathrm{O} & -0.922819 & 0.024003 & -1.933579 \\ \mathrm{O} & 1.122765 & -1.651487 & -0.730316 \\ \mathrm{O} & 1.321494 & 1.483843 & -0.764547 \\ \mathrm{O} & -1.477974 & -1.358720 & 0.590435 \\ \mathrm{O} & -1.307817 & 1.528360 & 0.567618 \\ \mathrm{O} & 0.676279 & -3.041096 & 1.927905 \\ \mathrm{O} & 0.839680 & -0.021448 & 1.765740 \\ \mathrm{O} & 1.053815 & 3.005757 & 1.824936 \\ \mathrm{~V} & 0.773679 & -0.073920 & -1.489675 \\ \mathrm{O} & 2.907797 & -0.168288 & -2.160739 \\ \mathrm{O} & -3.725182 & 0.237773 & -0.738915 \\ \mathrm{H} & 3.345800 & 0.588996 & -1.742006 \\ \mathrm{H} & 3.299705 & -0.968076 & -1.778425 \\ \mathrm{TI} & -1.951699 & 0.121376 & -0.388007 \\ \mathrm{TI} & 0.300321 & -1.568415 & 0.935801 \\ \mathrm{TI} & 0.505377 & 1.552436 & 0.887516 \\ \mathrm{H} & -4.343099 & -0.021139 & -1.428392 \\ \mathrm{H} & 0.247370 & -3.878134 & 2.128263 \\ \mathrm{H} & 0.963323 & 3.350224 & 2.717951\end{array}$

13

$\begin{array}{lrrr}0 & 0.038935 & 1.765416 & 1.417768 \\ 0 & 1.463889 & -0.840928 & 1.480064 \\ 0 & -1.494520 & -0.779224 & 1.483771 \\ 0 & 1.474417 & 0.768855 & -0.978903 \\ 0 & -1.441841 & 0.826950 & -0.978121 \\ 0 & 2.991438 & -1.872149 & -0.963702 \\ 0 & -0.037112 & -1.707587 & -0.920300 \\ 0 & -3.069983 & -1.745382 & -0.958148 \\ \text { V } & 0.003005 & 0.052870 & 1.831010 \\ O & 0.069421 & 3.464116 & -1.086181 \\ \text { TI } & 0.036469 & 1.780570 & -0.439310\end{array}$




$\begin{array}{lrrr}\text { TI } & -1.585122 & -0.889491 & -0.388083 \\ \mathrm{TI} & 1.544742 & -0.954538 & -0.391829 \\ \mathrm{H} & -3.961060 & -1.533819 & -1.251503 \\ \mathrm{H} & 3.902525 & -1.703361 & -1.221064 \\ \mathrm{H} & 0.118281 & 4.376741 & -0.787760\end{array}$

